\title{
Universal logarithmic behavior in microstate counting and the dual one-loop entropy of $\mathrm{AdS}_{4}$ black holes
}

\author{
Leopoldo A. Pando Zayas $\circledast^{1,2, *}$ and Yu Xin $\oplus^{3, \dagger}$ \\ ${ }^{1}$ Leinweber Center for Theoretical Physics, Randall Laboratory of Physics, The University of Michigan, \\ Ann Arbor, Michigan 48109-1120, USA \\ ${ }^{2}$ The Abdus Salam International Centre for Theoretical Physics, Strada Costiera 11, 34151 Trieste, Italy \\ ${ }^{3}$ Department of Applied Mathematics and Theoretical Physics, University of Cambridge, \\ Cambridge CB3 OWA, United Kingdom
}

(Received 18 October 2020; accepted 7 December 2020; published 4 January 2021)

\begin{abstract}
We numerically study the topologically twisted index of several three-dimensional supersymmetric field theories on a genus $g$ Riemann surface times a circle, $\Sigma_{g} \times S^{1}$. We show that for a large class of theories with leading term of the order $N^{3 / 2}$, where $N$ is generically the rank of the gauge group, there is a universal $\log$ arithmic correction of the form $\frac{g-1}{2} \log N$. We explain how this logarithmic subleading correction can be obtained as a one-loop effect on the dual supergravity theory for magnetically charged, asymptotically $\mathrm{AdS}_{4} \times M^{7}$ black holes for a large class of Sasaki-Einstein manifolds, $M^{7}$. The matching of the logarithmic correction relies on a generic cohomological property of $M^{7}$ and it is independent of the black hole charges. We argue that our supergravity results apply also to rotating, electrically charged asymptotically $\operatorname{AdS}_{4} \times$ $M^{7}$ black holes. We present explicitly the quiver gauge theories and the gravity side corresponding to $M^{7}=N^{0,1,0}, V^{5,2}$ and $Q^{1,1,1}$.

DOI: 10.1103/PhysRevD.103.026003
\end{abstract}

\section{INTRODUCTION}

One remarkable recent result in the context of the AdS/CFT correspondence is the microscopic understanding of the Bekenstein-Hawking entropy of a class of asymptotically $\mathrm{AdS}_{4}$ black holes. Benini, Hristov and Zaffaroni demonstrated in [1] that the topologically twisted index of Aharony-Bergman-Jafferis-Maldacena (ABJM) theory reproduces the entropy of the dual magnetically charged, asymptotically $\mathrm{AdS}_{4} \times S^{7}$ black holes.

Similar microscopic foundations via the topologically twisted index were provided for the corresponding macroscopic black hole entropy in different situations, including: dyonic black holes [2], black holes with hyperbolic horizons [3] and asymptotically $\mathrm{AdS}_{4}$ black holes in massive IIA supergravity [4,5]. Some interesting progress has also been reported in the higher dimensional context [6-10] and for $\mathrm{AdS}_{4}$ black holes embeddable in certain universal sectors of M2 [11] and M5 backgrounds [12,13] (see $[14,15]$ for reviews and a complete list of references for

\footnotetext{
1pandoz@umich.edu

†x328@cam.ac.uk
}

Published by the American Physical Society under the terms of the Creative Commons Attribution 4.0 International license. Further distribution of this work must maintain attribution to the author(s) and the published article's title, journal citation, and DOI. Funded by SCOAP . those developments). An analogous microscopic description, rooted in the superconformal index, has recently been presented for rotating, electrically charged $\mathrm{AdS}_{4}$ black holes $[16,17]$, including in a universal sector arising from wrapped M5 branes [18,19].

The robust agreement at the leading order inspired attempts to understand the topologically twisted index beyond the large $N$ limit with focus on the logarithmic corrections to the entropy on both sides of the correspondence $[20,21]$. The initial conclusion, however, was that more work was required and that Sen's quantum entropy formalism in its current formulation needed to be amended to also account for hair degrees of freedom away from the near-horizon region. Ultimately, precise agreement was found in [22] whose computation focused on the asymptotically $\mathrm{AdS}_{4}$ region of the black hole solution. Further successful matches of the logarithmic contributions were provided in the case of universally embedded black holes $[13,19]$. This subleading agreement motivates us to embark on a systematic exploration of a large class of models with the aim of demonstrating that the logarithmic correction is, indeed, quite universal. This is precisely one of the main results of this paper: an expression for the logarithmic corrections of the topologically twisted index for a large class of field theories on $\Sigma_{g} \times S^{1}$, which we find to be $\frac{g-1}{2} \log N$.

Let us describe two important previous results that make the journey to a universal logarithmic correction plausible. 
The first precedent pointing to the fact that the coefficient of $\log N$ in the topologically twisted index could be universal comes from a subleading analysis of the free energy of a large class of $3 \mathrm{~d}$ field theories. The exact partition function for a large class of Chern-Simons matter theories on $S^{3}$ can be computed using field theory localization and certain matrix model techniques, the answer can be succinctly written in terms of an Airy function [23]. In some cases the supergravity dual is known to be a background of M-theory on $\mathrm{AdS}_{4} \times M^{7}$, where $M^{7}$ is a Sasaki-Einstein seven-dimensional manifold. The universality of the logarithmic term in the free energy on $S^{3}$ established in [23] was beautifully elucidated from the dual supergravity point of view in [24] and shown to depend on some mild cohomological properties of the seven-dimensional manifold $M^{7}$. Some of our arguments in this manuscript mimic that analysis closely. The other important source of inspiration for us is a group of works that established a leading order in $N$ relationship between the free energy on $S^{3}$ and the topologically twisted index on $S^{2} \times S^{1}$ presented and developed in $[25,26]$. There is a formal background that arguably provides a rigorous basis for relations among the free energy on $S^{3}$ and the topologically twisted index in $\Sigma_{g} \times$ $S^{1}$ [12,27-29] but we were particularly inspired by the two developments mentioned above. In this manuscript we effectively ask the questions of whether there is a relationship between the free energy on $S^{3}$ and the topologically twisted index in $\Sigma_{g} \times S^{1}$ beyond the leading order in $N$ and, in particular, whether we can establish the universality of the logarithmic in $N$ correction. We are not able to answer the broader question of the relationship between the free energy on $S^{3}$ and the topologically twisted index beyond the large $N$ limit but we present strong numerical evidence in favor of a universal logarithmic correction in the topologically twisted index very similar to the universality of the logarithmic term for the free energy.

The rest of the manuscript is organized as follows. We start in Sec. II by briefly reviewing the topologically twisted index in general and its form for the ABJM theory. Sections III, IV and V are devoted to extensive numerical evaluations of the topologically twisted index for the $3 \mathrm{~d}$ Chern-Simons matter theories dual to M-theory on $\mathrm{AdS}_{4} \times$ $M^{7}$ for $M^{7}=N^{0,1,0}, V^{5,2}, Q^{1,1,1}$, respectively. We discuss the one-loop gravity computation dual to the universal result in Sec. VI. We conclude in Sec. VII where we also point to a number of interesting, in our opinion, open problems.

\section{THE TOPOLOGICALLY TWISTED INDEX FOR GENERIC $\mathcal{N}=2$ THEORIES}

In this section we will briefly review the construction and structure of the topologically twisted index for $3 \mathrm{~d} \mathcal{N}=2$ supersymmetric theories. The topologically twisted index for three dimensional $\mathcal{N}=2$ field theories was defined in [30] (see other related works [25,26,31-33]) by evaluating the supersymmetric partition function on $S^{1} \times S^{2}$ with a topological twist on $S^{2}$. One considers a 3d theory, usually containing Yang-Mills, $\mathcal{L}_{\mathrm{YM}}$, and Chern-Simons, $\mathcal{L}_{C S}$, interactions on $S^{2} \times S^{1}$ with metric and background field given as

$d s^{2}=R^{2}\left(d \theta^{2}+\sin ^{2} \theta d \phi^{2}\right)+\beta^{2} d t^{2}, \quad A^{R}=\frac{1}{2} \cos \theta d \phi$.

There is typically a set of flavor symmetries characterized by Cartan-valued magnetic fluxes:

$$
J^{f}=\frac{1}{2 \pi} \int_{S^{2}} F^{f}=\mathfrak{n} .
$$

With these magnetic fluxes one associates flavor fugacities $y=\exp \left[i\left(A_{t}^{f}+i \beta \sigma^{f}\right)\right]$, where the constant potential $A_{t}^{f}$ is a flat connection for the flavor symmetry and $\sigma^{f}$ is a real mass for the three-dimensional field theory. Similarly the fugacities for the dynamical fields are $x=\exp \left[i\left(A_{t}+\right.\right.$ $i \beta \sigma)]$, where $A_{t}$ runs over the maximal torus of the gauge group and $\sigma$ over the corresponding Cartan subalgebra.

The topologically twisted index generically takes the from

$$
Z(\mathfrak{n}, y)=\frac{1}{|W|} \sum_{\mathfrak{m} \in \Gamma_{\mathfrak{h}}} \oint_{\mathcal{C}} Z_{\text {int }}(x, y ; \mathfrak{m}, \mathfrak{n}) .
$$

There is an algorithmic way of constructing $Z_{\text {int }}$ depending on the field content of the theory. Let us define the building blocks that go into $Z_{\text {int }}$. For a chiral multiplet

$$
Z_{1 \text {-loop }}^{\text {chiral }}=\prod_{\rho \in \Re}\left(\frac{x^{\rho / 2} y^{\rho_{f} / 2}}{1-x^{\rho} y^{\rho_{f}}}\right)^{\rho(\mathfrak{m})+\rho_{f}(\mathfrak{n})-q+1}
$$

where $\mathfrak{R}$ is the representation of the gauge group $G, \rho$ denote the corresponding weights, $q$ is the R-charge of the field and $\rho_{f}$ is the weight of the multiplet under the flavor symmetry group. For the gauge multiplet one has

$$
Z_{1 \text {-loop }}^{\text {gauge }}=\prod_{\alpha \in G}\left(1-x^{\alpha}\right)(i d u)^{r},
$$

where $r$ is the rank of the gauge group and $\alpha$ donate the roots of $G$. We also use $u=A_{t}+i \beta \sigma$ which lives on the complexified Cartan subalgebra, essentially, $x=e^{i u}$.

The only classical contribution to $Z_{\text {int }}$ comes from the Chern-Simons term and takes the form 


$$
Z_{\text {class }}^{C S}=x^{k \mathfrak{m}},
$$

where $k$ is the Chern-Simons level and $\mathfrak{m}$ is the magnetic flux taking values in the co-root latice $\Gamma_{\mathfrak{h}}$ of the gauge group. There is also the contribution of a $U(1)$ topological symmetry with holonomy $\xi=e^{i z}$ and flux $\mathrm{t}$ :

$$
Z_{\text {class }}^{\text {top }}=x^{\mathrm{t}} \xi^{\mathfrak{m}} \text {. }
$$

With these ingredients one has that the index takes the general form

$$
\begin{aligned}
Z(\mathfrak{n}, y)= & \frac{1}{|W|} \sum_{\mathfrak{m} \in \Gamma_{\mathfrak{h}}} \oint_{\mathcal{C}} \prod_{\text {Cartan }}\left(\frac{d x}{2 \pi i x} x^{k \mathfrak{m}}\right) \prod_{\alpha \in G}\left(1-x^{\alpha}\right) \\
& \times \prod_{I} \prod_{\rho_{I} \in \Re_{I}}\left(\frac{x^{\rho_{I} / 2} y_{I}^{1 / 2}}{1-x^{\rho_{I}} y_{I}}\right)^{\rho_{I}(\mathfrak{m})-\mathfrak{n}_{I}+1},
\end{aligned}
$$

where $\alpha$ are the roots of $G, \rho_{I}$ are the weights of the representation $\mathfrak{R}_{I}$ and $\mathfrak{m}$ are gauge magnetic fluxes living in the co-root lattice $\Gamma_{\mathfrak{h}}$.

The index depends on a choice of fugacities $y_{I}$ for the flavor group and a choice of integer magnetic charges $\mathfrak{n}_{I}$ for the R-symmetry of the theory. Both $y_{I}$ and $\mathfrak{n}_{I}$ are parameterized by the global symmetries of the theory. Each monomial term $W$ in the superpotential imposes a constraint:

$$
\prod_{I \in W} y_{I}=1, \quad \prod_{I \in W} \mathfrak{n}_{I}=2
$$

where the product and sum are restricted to the fields entering in $W$. These constraints are called the marginality conditions of the superpotential.

After summing over the magnetic fluxes, $\mathfrak{m}$, in Eq. (2.8), one obtains an expression for the index whose poles are located at positions determined by the following BetheAnsatz like expression

$$
\exp \left(i \operatorname{sign}\left(k_{a}\right) B_{i}^{(a)}\right)=1 .
$$

For the class of theories we are interested in this manuscript it is convenient to consider some representations explicitly. The ingredients in the topologically twisted index that we will require are

(i) The Vandermonde determinant contributes to the logarithm of the index as

$$
\begin{aligned}
\log & \prod_{i \neq j}\left(1-\frac{x_{i}^{(a)}}{x_{j}^{(a)}}\right) \\
= & \log \prod_{i<j}\left(1-\frac{x_{j}^{(a)}}{x_{i}^{(a)}}\right)^{2}\left(-\frac{x_{i}^{(a)}}{x_{j}^{(a)}}\right) \\
= & i \sum_{i<j}^{N}\left(u_{i}^{(a)}-u_{j}^{(a)}+\pi\right)-2 \sum_{i<j}^{N} \operatorname{Li}_{1}\left(e^{i\left(u_{j}^{(a)}-u_{i}^{(a)}\right)}\right) .
\end{aligned}
$$

(ii) The topological symmetry contributes as

$$
i \sum_{i=1}^{N} u_{i}^{(a)} \mathbf{t}_{a},
$$

where $\mathbf{t}_{a}$ is the flux of the $U(1)_{a}$ topological symmetry.

(iii) A bifundamental chiral multiplet transforming in $(\overline{\mathbf{N}}, \mathbf{N})$ of $U(N)_{a} \times U(N)_{b}$ with magnetic flux $\mathfrak{n}_{(b, a)}$ and chemical potential $\Delta_{(b, a)}$ contributes as

$$
\begin{aligned}
& \prod_{i=1}^{N}\left(\frac{x_{i}^{(a)}}{x_{i}^{(b)}}\right)^{\frac{1}{2}\left(\mathfrak{n}_{(b, a)}-1\right)}\left(1-y_{(b, a)} \frac{x_{i}^{(b)}}{x_{i}^{(a)}}\right)^{\mathfrak{n}_{(b, a)}-1} \\
& \quad \times \prod_{i<j}^{N}(-1)^{\mathfrak{n}_{(b, a)}-1}\left(\frac{x_{i}^{(a)} x_{i}^{(b)}}{x_{j}^{(a)} x_{j}^{(b)}}\right)^{\frac{1}{2}\left(\mathfrak{n}_{(b, a)}-1\right)} \\
& \quad \times\left(1-y_{(b, a)} \frac{x_{j}^{(b)}}{x_{i}^{(a)}}\right)^{\mathfrak{n}_{(b, a)}-1}\left(1-y_{(b, a)}^{-1} \frac{x_{j}^{(a)}}{x_{i}^{(b)}}\right)^{\mathfrak{n}_{(b, a)}-1} .
\end{aligned}
$$

(iv) Fundamental and antifundamental fields contribute as

$$
\begin{aligned}
\log & \prod_{i=1}^{N} \prod_{\substack{\text { anti-fundamental } \\
a}}\left(x_{i}^{(a)}\right)^{\frac{1}{2}\left(\tilde{\mathfrak{n}}_{a}-1\right)}\left[1-\tilde{y}_{a}\left(x_{i}^{(a)}\right)^{-1}\right]^{\tilde{\mathfrak{n}}_{a}-1} \\
& \times \prod_{\substack{\text { fundamental } \\
a}}\left(x_{i}^{(a)}\right)^{\frac{1}{2}\left(\mathfrak{n}_{a}-1\right)}\left[1-y_{a}^{-1}\left(x_{i}^{(a)}\right)^{-1}\right]^{\mathfrak{n}_{a}-1} .
\end{aligned}
$$

(v) As anticipated above, the contour integration is best expressed in terms of the position of the poles, given by the BA equation in terms of $B_{i}$. In taking the residues from one set of variables $x_{i}$ to another $B_{i}$, we incur a Jacobian denoted by $\mathbb{B}$ :

$$
\begin{aligned}
\mathbb{B} & =\frac{\partial\left(e^{i B_{j}^{(a)}}, e^{i B_{j}^{(b)}}\right)}{\partial\left(\log x_{l}^{(a)}, \log x_{l}^{(b)}\right)} \\
& =\left(\begin{array}{cc}
x_{l}^{(a)} \frac{e^{i B_{j}^{(a)}}}{\partial x_{l}^{(a)}} & x_{l}^{(b)} \frac{e_{j}^{i B_{j}^{(a)}}}{\partial x_{l}^{(b)}} \\
x_{l}^{(a)} \frac{e^{i B_{j}^{(b)}}}{\partial x_{l}^{(a)}} & x_{l}^{(b)} \frac{e_{j}^{i B_{j}^{(b)}}}{\partial x_{l}^{(b)}}
\end{array}\right)_{2 N \times 2 N},
\end{aligned}
$$


where

$$
\begin{aligned}
\exp \left(i \operatorname{sign}\left(k_{a}\right) B_{i}^{(a)}\right)= & \left(\xi^{(a)}\right)^{\operatorname{sign}\left(k_{a}\right)}\left(x_{i}^{(a)}\right)^{k_{a}} \prod_{\substack{\text { birundamentals } \\
(b, a) \text { and }(a, b)}} \prod_{j=1}^{N} \frac{\sqrt{\frac{x_{i}^{(a)}}{x_{j}^{(b)}} y_{(a, b)}}}{1-\frac{x_{i}^{(a)}}{x_{j}^{(b)}} y_{(a, b)}} \frac{1-\frac{x_{j}^{(b)}}{x_{i}^{(a)}} y_{(b, a)}}{\sqrt{\frac{x_{j}^{(b)}}{x_{i}^{(a)}} y_{(b, a)}}} \\
& \times \prod_{\text {fundamentals }} \frac{\sqrt{x_{i}^{(a)} y_{a}}}{1-x_{i}^{(a)} y_{a}} \prod_{a \text { antifundamentals }} \frac{1-\frac{1}{x_{i}^{(a)}} \tilde{y}_{a}}{\sqrt{\frac{1}{x_{i}^{(a)}} \tilde{y}_{a}}} .
\end{aligned}
$$

\section{A. The topologically twisted index}

Explicitly, the general expression of the index is

$$
\begin{aligned}
& Z=\frac{1}{(N !)^{|G|}} \sum_{\left\{\mathfrak{m} ; \mathfrak{m} \in \mathbb{Z}^{N}\right\}} \int_{\mathcal{C}} \prod_{a=1}^{|G|}\left[\prod_{i=1}^{N} \frac{d x_{i}^{(a)}}{2 \pi i x_{i}^{(a)}}\left(x_{i}^{(a)}\right)^{k_{a} \mathfrak{m}_{i}^{(a)}+\mathfrak{t}^{(a)}}\left(\xi^{(a)}\right)^{\operatorname{sign}\left(k_{a}\right) \mathfrak{m}_{i}^{(a)}} \times \prod_{i \neq j}^{N}\left(1-\frac{x_{i}^{(a)}}{x_{j}^{(a)}}\right)\right] \\
& \times \prod_{i, j=1}^{N}\left[\prod_{\substack{\text { bifundamentals } \\
(b, a) \text { and }(a, b)}}\left(\frac{\sqrt{\frac{x_{i}^{(a)}}{x_{j}^{(b)}} y_{(a, b)}}}{1-\frac{x_{i}^{(a)}}{x_{j}^{(b)}} y_{(a, b)}}\right)^{\mathfrak{m}_{i}^{(a)}-\mathfrak{m}_{j}^{(b)}-\mathfrak{n}_{(a, b)}+1}\left(\frac{\sqrt{\frac{x_{j}^{(b)}}{x_{i}^{(a)}} y_{(b, a)}}}{1-\frac{x_{j}^{(b)}}{x_{i}^{(a)}} y_{(b, a)}}\right)^{\mathfrak{m}_{j}^{(b)}-\mathfrak{m}_{i}^{(a)}-\mathfrak{n}_{(b, a)}+1}\right. \\
& \left.\times \prod_{\substack{\text { adjoints } \\
(a, a)}}\left(\frac{\sqrt{\frac{x_{i}^{(a)}}{x_{j}^{(a)}} y_{(a, a)}}}{1-\frac{x_{i}^{(a)}}{x_{j}^{(a)}} y_{(a, a)}}\right)^{\mathfrak{m}_{i}^{(a)}-\frac{1}{2} \mathfrak{n}_{(a, a)}+\frac{1}{2}}\left(\frac{\sqrt{\frac{x_{j}^{(a)}}{x_{i}^{(a)}} y_{(a, a)}}}{1-\frac{x_{j}^{(a)}}{x_{i}^{(a)}} y_{(a, a)}}\right)^{-\mathfrak{m}_{i}^{(a)}-\frac{1}{2} \mathfrak{n}_{(a, a)}+\frac{1}{2}}\right] \\
& \times \prod_{i=1}^{N}\left[\prod_{\substack{\text { fundamentals } \\
a}}\left(\frac{\sqrt{x_{i}^{(a)} y_{a}}}{1-x_{i}^{(a)} y_{a}}\right)^{\mathfrak{m}_{i}^{(a)}-\mathfrak{n}_{a}+1} \prod_{\substack{\text { antifundamentals } \\
a}}\left(\frac{\sqrt{\frac{1}{x_{i}^{(a)}} \tilde{y}_{a}}}{1-\frac{1}{x_{i}^{(a)}} \tilde{y}_{a}}\right)^{-\mathfrak{m}_{i}^{(a)}-\tilde{\mathfrak{n}}_{a}+1}\right] .
\end{aligned}
$$

The sum over magnetic fluxes is effectively a geometric sum introducing a large cutoff $M$ and the index takes the form

$$
\begin{aligned}
& Z=\frac{1}{(N !)^{|G|}} \int_{\mathcal{C}} \prod_{a=1}^{|G|}\left[\prod_{i=1}^{N} \frac{d x_{i}^{(a)}}{2 \pi i x_{i}^{(a)}}\left(x_{i}^{(a)}\right)^{\mathfrak{t}^{(a)}} \times \prod_{i \neq j}^{N}\left(1-\frac{x_{i}^{(a)}}{x_{j}^{(a)}}\right) \times \prod_{i=1}^{N} \frac{\left(e^{i B_{i}^{(a)}}\right)^{M}}{e^{i B_{i}^{(a)}}-1}\right] \\
& \times \prod_{i, j=1}^{N}\left[\prod_{\substack{\text { bifundamentals } \\
(b, a) \text { and }(a, b)}}\left(\frac{\sqrt{\frac{x_{i}^{(a)}}{x_{j}^{(b)}} y_{(a, b)}}}{1-\frac{x_{i}^{(a)}}{x_{j}^{(b)}} y_{(a, b)}}\right)^{1-\mathfrak{n}_{(a, b)}}\left(\frac{\sqrt{\frac{x_{j}^{(b)}}{x_{i}^{(a)}} y_{(b, a)}}}{1-\frac{x_{j}^{(b)}}{x_{i}^{(a)}} y_{(b, a)}}\right)^{1-\mathfrak{n}_{(b, a)}}\right. \\
& \left.\times \prod_{\substack{\text { adjoints } \\
(a, a)}}\left(\frac{\sqrt{y_{(a, a)}}}{1-\frac{x_{j}^{(a)}}{x_{i}^{(a)}} y_{(a, a)}}\right)^{1-\mathfrak{n}_{(a, a)}}\right] \\
& \times \prod_{i=1}^{N}\left[\prod_{\substack{\text { fundamentals } \\
a}}\left(\frac{\sqrt{x_{i}^{(a)} y_{a}}}{1-x_{i}^{(a)} y_{a}}\right)^{\left(1-\mathfrak{n}_{a}\right)} \prod_{\substack{\text { antifundamentals } \\
a}}\left(\frac{\sqrt{\frac{1}{x_{i}^{(a)}} \tilde{y}_{a}}}{1-\frac{1}{x_{i}^{(a)}} \tilde{y}_{a}}\right)^{\left(1-\tilde{\mathfrak{n}}_{a}\right)}\right] .
\end{aligned}
$$

This is precisely the main expression we will consider. 


\section{B. Bethe Ansatz potential}

$$
y_{I}=e^{i \Delta_{I}}, \xi^{(a)}=e^{i \Delta_{m}^{(a)}},
$$

An alternative way to package the information in the index is to consider the so-called Bethe-Ansatz potential, $\mathcal{V}$. The Bethe-Ansatz potential succinctly summarizes the Bethe-Ansatz equations. For the representations we will consider in this manuscript it is possible to write

$$
\mathcal{V}=\mathcal{V}^{C S}+\mathcal{V}^{\text {bi-fund }}+\mathcal{V}^{\text {adjoint }}+\mathcal{V}^{\text {(anti-)fund }} \text {. }
$$

the Bethe potential is given by

Introducing chemical potentials:

$$
\begin{aligned}
\mathcal{V}^{\mathrm{bi}-\text { fund }}= & \sum_{\substack{\text { bifundamentals } \\
(b, a) \text { and }(a, b)}} \sum_{i, j=1}^{N}\left[\operatorname{Li}_{2}\left(e^{i\left(u_{j}^{(b)}-u_{i}^{(a)}+\Delta_{(b, a)}\right)}\right)-\mathrm{Li}_{2}\left(e^{i\left(u_{j}^{(b)}-u_{i}^{(a)}-\Delta_{(a, b)}\right)}\right)\right] \\
& +\operatorname{Arg}\left[\operatorname { e x p } \left(i \left(-\frac{1}{2} \operatorname{Arg}\left[\exp \left(i \sum_{\substack{\text { bifindanentals } \\
\text { abala and }(a, b)}}\left(\Delta_{(b, a)}+\Delta_{(a, b)}\right)\right)\right]\right.\right.\right. \\
& \left.\left.\left.+\sum_{\substack{\text { bifundanentals } \\
(b, a) \text { and }(a, b)}} \pi\right)\right)\right] \sum_{i, j=1}^{N}\left(u_{j}^{(b)}-u_{i}^{(a)}\right),
\end{aligned}
$$

and

$$
\begin{aligned}
\mathcal{V}^{\text {(anti-)fund }}= & \sum_{i=1}^{N}\left[\sum_{\text {antifundamental }}^{a} \operatorname{Li}_{2}\left(e^{i\left(-u_{i}^{(a)}+\tilde{\Delta}_{a}\right)}\right)-\sum_{\substack{\text { fundamental } \\
a}} \operatorname{Li}_{2}\left(e^{i\left(-u_{i}^{(a)}-\Delta_{a}\right)}\right)\right] \\
& +\frac{1}{2} \sum_{i=1}^{N}\left[\sum_{\substack{\text { antifundamental } \\
a}}\left(\tilde{\Delta}_{a}-\pi\right) u_{i}^{(a)}+\sum_{\substack{\text { fundamental } \\
a}}\left(\Delta_{a}-\pi\right) u_{i}^{(a)}\right] \\
& -\frac{1}{4} \sum_{i=1}^{N}\left[\sum_{\substack{\text { antifundamental } \\
a}}\left(u_{i}^{(a)}\right)^{2}-\sum_{\substack{\text { fundamental } \\
a}}\left(u_{i}^{(a)}\right)^{2}\right] .
\end{aligned}
$$

Adjoint fields are treated as a special case of bifundamentals with $\Delta_{(b, a)}=\Delta_{(a, b)}=\Delta_{(a, a)}$ and an explicit factor of $1 / 2$. The second term in the bifundamental potential Eq. (2.22) is a little different from (A.10) in [25] for consistency with the potential in the ABJM theory in [1]. This difference will only translate all of the eigenvalues along the real axis by a constant depending on $N$, which has no effect on the final result of Re $\log Z$. Under the choice of the second term in Eq. (2.22), the eigenvalues for different values of $N$ will be concentrated without any translation along the real axis.

\section{The topologically twisted index of ABJM beyond the large $N$ limit}

As a way of giving the above general description of the topologically twisted index some concrete context, let us consider the ABJM theory [34] which is a three-dimensional supersymmetric Chern-Simons-matter theory with gauge group $U(N)_{k} \times U(N)_{-k}$ (the subscripts denote the Chern-
Simons levels) and matter in bifundamental representations. A simple representation of the theory is via standard $\mathcal{N}=2$ notation in terms of the quiver diagram below:

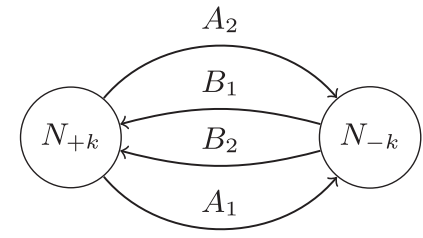

The superpotential of the theory is

$$
W=\operatorname{Tr}\left[A_{1} B_{1} A_{2} B_{2}-A_{1} B_{2} A_{2} B_{1}\right] .
$$

There are a total of four $U(1)$ gauge fields from the Cartan of the $S O(8)$ R-symmetry, with corresponding charges $\mathfrak{n}_{a}$ satisfying the supersymmetry constraint $\sum \mathfrak{n}_{a}=2$. The expression for the topologically twisted index, before summing over the magnetic fluxes $\mathfrak{m}$ takes the form 


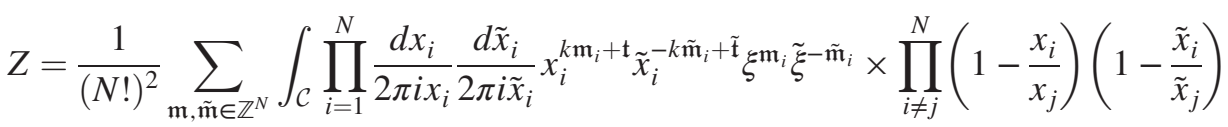

$$
\begin{aligned}
& \times \prod_{i, j=1}^{N} \prod_{a=1,2}\left(\frac{\sqrt{\frac{x_{i}}{\tilde{x}_{j}} y_{a}}}{1-\frac{x_{i}}{\tilde{x}_{j}} y_{a}}\right)^{\mathfrak{m}_{i}-\tilde{\mathfrak{m}}_{j}-\mathfrak{n}_{a}+1} \prod_{b=3,4}\left(\frac{\sqrt{\frac{\tilde{x}_{j}}{x_{i}} y_{b}}}{1-\frac{\tilde{x}_{j}}{x_{i}} y_{b}}\right)^{\tilde{\mathfrak{m}}_{j}-\mathfrak{m}_{i}-\mathfrak{n}_{b}+1} .
\end{aligned}
$$

Performing the summation over magnetic fluxes introducing a large cutoff $M$ we get

$$
\begin{aligned}
Z= & \frac{1}{(N !)^{2}} \int_{\mathcal{C}} \prod_{i=1}^{N} \frac{d x_{i}}{2 \pi i x_{i}} \frac{d \tilde{x}_{i}}{2 \pi i \tilde{x}_{i}} \prod_{i \neq j}^{N}\left(1-\frac{x_{i}}{x_{j}}\right)\left(1-\frac{\tilde{x}_{i}}{\tilde{x}_{j}}\right) \times \prod_{i, j=1}^{N} \prod_{a=1,2}\left(\frac{\sqrt{\frac{x_{i}}{\tilde{x}_{j}} y_{a}}}{1-\frac{x_{i}}{\tilde{x}_{j}} y_{a}}\right)^{1-\mathfrak{n}_{a}} \prod_{b=3,4}\left(\frac{\sqrt{\frac{\tilde{x}_{j}}{x_{i}} y_{b}}}{1-\frac{\tilde{x}_{j}}{x_{i}} y_{b}}\right)^{1-\mathfrak{n}_{b}} \\
& \times \prod_{i=1}^{N} \frac{\left(e^{i B_{i}}\right)^{M}}{e^{i B_{i}}-1} \prod_{j=1}^{N} \frac{\left(e^{i \tilde{B}_{j}}\right)^{M}}{e^{i \tilde{B}_{j}}-1}
\end{aligned}
$$

The topologically twisted index for ABJM theory was worked out in [1], and reduces to the evaluation of the partition function

$$
Z\left(y_{a}, \mathfrak{n}_{a}\right)=\prod_{a=1}^{4} y_{a}^{-\frac{1}{2} N^{2} \mathfrak{n}_{a}} \sum_{I \in B A E} \frac{1}{\operatorname{det} \mathbb{B}} \frac{\prod_{i=1}^{N} x_{i}^{N} \tilde{x}_{i}^{N} \prod_{i \neq j}\left(1-\frac{x_{i}}{x_{j}}\right)\left(1-\frac{\tilde{x}_{i}}{\tilde{x}_{j}}\right)}{\prod_{i, j=1}^{N} \prod_{a=1,2}\left(\tilde{x}_{j}-y_{a} x_{i}\right)^{1-\mathfrak{n}_{a}} \prod_{a=3,4}\left(x_{i}-y_{a} \tilde{x}_{j}\right)^{1-\mathfrak{n}_{a}}},
$$

where $y_{a}$ are the corresponding fugacities. The summation is over all solutions $I$ of the "Bethe Ansatz equations" (BAE) $e^{i B_{i}}=e^{i \tilde{B}_{i}}=1$ modulo permutations, where

$$
\begin{aligned}
e^{i B_{i}} & =x_{i}^{k} \prod_{j=1}^{N} \frac{\left(1-y_{3} \frac{\tilde{x}_{j}}{x_{i}}\right)\left(1-y_{4} \frac{\tilde{x}_{j}}{x_{i}}\right)}{\left(1-y_{1}^{-1} \frac{\tilde{x}_{j}}{x_{i}}\right)\left(1-y_{2}^{-1} \frac{\tilde{x}_{j}}{x_{i}}\right)}, \\
e^{i \tilde{B}_{j}} & =\tilde{x}_{j}^{k} \prod_{i=1}^{N} \frac{\left(1-y_{3} \frac{\tilde{x}_{j}}{x_{i}}\right)\left(1-y_{4} \frac{\tilde{x}_{j}}{x_{i}}\right)}{\left(1-y_{1}^{-1} \frac{\tilde{x}_{j}}{x_{i}}\right)\left(1-y_{2}^{-1} \frac{\tilde{x}_{j}}{x_{i}}\right)} .
\end{aligned}
$$

Here $k$ is the Chern-Simons level, and the two sets of variables $\left\{x_{i}\right\}$ and $\left\{\tilde{x}_{j}\right\}$ arise from the $U(N)_{k} \times U(N)_{-k}$ structure of ABJM theory. Finally, the $2 N \times 2 N$ matrix $\mathbb{B}$ is the Jacobian relating the $\left\{x_{i}, \tilde{x}_{j}\right\}$ variables to the $\left\{e^{i B_{i}}, e^{i \tilde{B}_{j}}\right\}$ variables

$$
\mathbb{B}=\left(\begin{array}{ll}
x_{l} \frac{\partial e^{i B_{j}}}{\partial x_{l}} & \tilde{x}_{l} \frac{\partial e^{i B_{j}}}{\partial \tilde{x}_{l}} \\
x_{l} \frac{\partial e^{i \tilde{B}_{j}}}{\partial x_{l}} & \tilde{x}_{l} \frac{\partial e^{i \tilde{B}_{j}}}{\partial \tilde{x}_{l}}
\end{array}\right)
$$

It is convenient to introduce the chemical potentials $\Delta_{a}$ according to $y_{a}=e^{i \Delta_{a}}$ and furthermore perform a change of variables $x_{i}=e^{i u_{i}}, \tilde{x}_{j}=e^{i \tilde{u}_{j}}$. In this case, the BAE become

$$
\begin{aligned}
0= & k u_{i}-i \sum_{j=1}^{N}\left[\sum_{a=3,4} \log \left(1-e^{i\left(\tilde{u}_{j}-u_{i}+\Delta_{a}\right)}\right)\right. \\
& \left.-\sum_{a=1,2} \log \left(1-e^{i\left(\tilde{u}_{j}-u_{i}-\Delta_{a}\right)}\right)\right]-2 \pi n_{i}, \\
0= & k \tilde{u}_{j}-i \sum_{i=1}^{N}\left[\sum_{a=3,4} \log \left(1-e^{i\left(\tilde{u}_{j}-u_{i}+\Delta_{a}\right)}\right)\right. \\
& \left.-\sum_{a=1,2} \log \left(1-e^{i\left(\tilde{u}_{j}-u_{i}-\Delta_{a}\right)}\right)\right]-2 \pi \tilde{n}_{j} .
\end{aligned}
$$

The topologically twisted index is evaluated by first solving these equations for $\left\{u_{i}, \tilde{u}_{j}\right\}$, and then inserting the resulting solution into the partition function Eq. (2.28). This procedure was carried out in [1] in the large $N$ limit with $k=1$ by introducing the parametrization:

$$
\begin{aligned}
& u_{i}=i N^{1 / 2} t_{i}+\pi-\frac{1}{2} \delta v\left(t_{i}\right), \\
& \tilde{u}_{i}=i N^{1 / 2} t_{i}+\pi+\frac{1}{2} \delta v\left(t_{i}\right),
\end{aligned}
$$

where we have further made use of reflection symmetry about $\pi$ along the real axis. In the large $N$ limit, the eigenvalue distribution becomes continuous, and the set $\left\{t_{i}\right\}$ may be described by an eigenvalue density $\rho(t)$.

The leading order solution for $\rho(t)$ and $\delta v(t)$ was worked out in [1], and the resulting partition function exhibits the expected $N^{3 / 2}$ scaling of ABJM theory: 


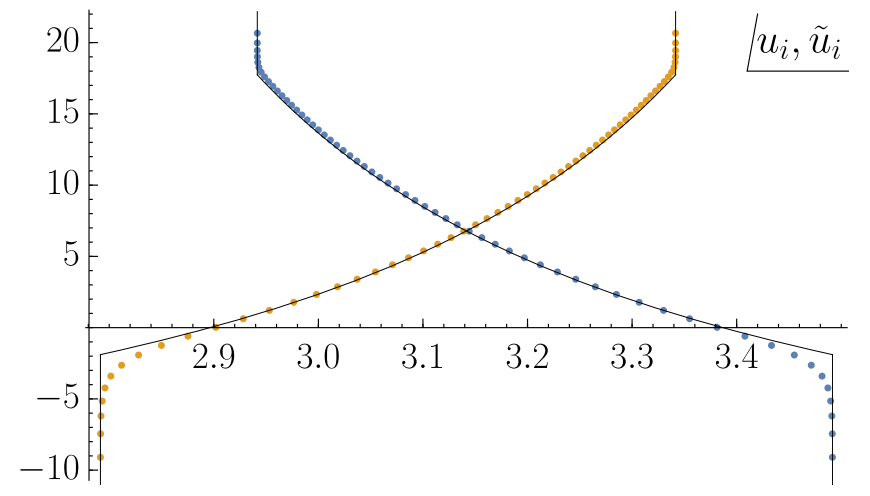

FIG. 1. The solution to the BAE for $\Delta_{a}=\{0.4,0.5,0.7,2 \pi-$ $1.6\}$ and $N=60$. The solid lines correspond to the leading order expression obtained in [1].

$$
\operatorname{Re} \log Z_{0}=-\frac{N^{3 / 2}}{3} \sqrt{2 \Delta_{1} \Delta_{2} \Delta_{3} \Delta_{4}} \sum_{a} \frac{\mathfrak{n}_{a}}{\Delta_{a}} .
$$

\section{Evaluation of the index beyond the leading order in $N$}

Given that the BA approach provides the exact answer in $N$ a numerical study of this topologically twisted index was performed in [20] and established that presence of a logarithmic correction of the form $-\frac{1}{2} \log N$. In this manuscript we take [20] as a blueprint and extend that analysis to a number of models with the goal of determining whether this logarithmic contribution is universal; we find that, indeed, it is. Let us thus briefly review the main result and some of the techniques of [20].

In the ABJM context, one expects the subleading behavior of the index to have the form

$$
\begin{aligned}
\operatorname{Re} \log Z= & \operatorname{Re} \log Z_{0}+f_{1}\left(\Delta_{a}, \mathfrak{n}_{a}\right) N^{1 / 2} \\
& +f_{2}\left(\Delta_{a}, \mathfrak{n}_{a}\right) \log N+f_{3}\left(\Delta_{a}, \mathfrak{n}_{a}\right)+\mathcal{O}\left(N^{-1 / 2}\right),
\end{aligned}
$$

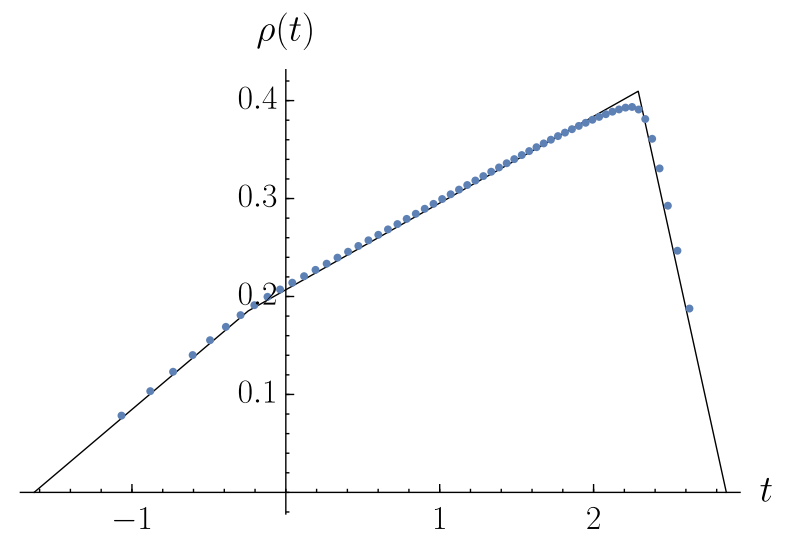

where the functions $f_{1}, f_{2}$ and $f_{3}$ are linear in the magnetic fluxes $n_{a}$.

Let us quote some results from [20] where the numerical solution for the eigenvalues $u_{i}$ and $\tilde{u}_{i}$ for $\Delta_{a}=\{0.4$, $0.5,0.7,2 \pi-1.6\}$ and $N=60$ is shown in Figure 1 quoted from [20]. The corresponding eigenvalue density $\rho(t)$ and function $\delta v(t)$ are shown in Figure 2 quoted from [20].

Once the eigenvalues are obtained, it is then simply a matter of numerically evaluating the index Eq. (2.28) on the solution to the BAE. For a given set of chemical potentials $\Delta_{a}$, we compute $\log Z$ for a range of $N$. We then subtract out the leading behavior Eq. (2.33) and decompose the residuals into a sum of four independent terms:

$\operatorname{Re} \log Z=\operatorname{Re} \log Z_{0}+A+B_{1} \mathfrak{n}_{1}+B_{2} \mathfrak{n}_{2}+B_{3} \mathfrak{n}_{3}$,

where we have used the condition $\sum_{a} \mathfrak{n}_{a}=2$. At this stage, we then perform a linear least-squares fit of $A$ and $B_{a}$ to the function

$$
\begin{aligned}
f(N)= & f_{1} N^{1 / 2}+f_{2} \log N+f_{3}+f_{4} N^{-1 / 2} \\
& +f_{5} N^{-1}+f_{6} N^{-3 / 2} .
\end{aligned}
$$

The results of the numerical fit are presented in Table I quoted from [20] whose main result is that the numerical evidence points to the coefficient of the $\log N$ term being exactly $-1 / 2$. We thus have

$$
\begin{aligned}
\operatorname{Re} \log Z= & -\frac{N^{3 / 2}}{3} \sqrt{2 \Delta_{1} \Delta_{2} \Delta_{3} \Delta_{4}} \sum_{a} \frac{\mathfrak{n}_{a}}{\Delta_{a}} \\
& +N^{1 / 2} f_{1}\left(\Delta_{a}, \mathfrak{n}_{a}\right)-\frac{1}{2} \log N \\
& +f_{3}\left(\Delta_{a}, \mathfrak{n}_{a}\right)+\mathcal{O}\left(N^{-1 / 2}\right),
\end{aligned}
$$

where $f_{1}$ and $f_{3}$ remain to be determined.

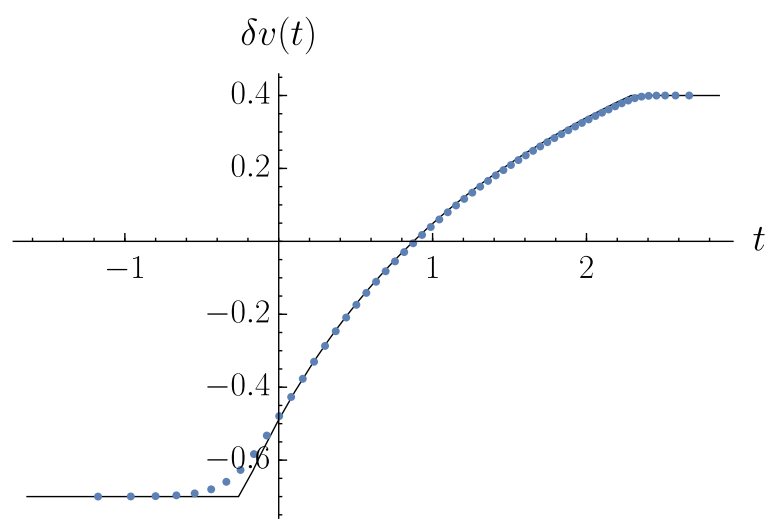

FIG. 2. The eigenvalue density $\rho(t)$ and the function $\delta v(t)$ for $\Delta_{a}=\{0.4,0.5,0.7,2 \pi-1.6\}$ and $N=60$, compared with the leading order expression. 
TABLE I. (ABJM) Numerical fit for Re $\log Z=\operatorname{Re} \log Z_{0}+f_{1} N^{1 / 2}+f_{2} \log N+f_{3}+\cdots$. The values of $N$ used in the fit range from 50 to $N_{\max }$ in steps of 10 where $N_{\max }=290,150,190,120$ for the four cases, respectively. We made use of the fact that the index is independent of the magnetic fluxes when performing the fit for the special case $\left(\Delta_{a}=\{\pi / 2, \pi / 2, \pi / 2, \pi / 2\}\right)$.

\begin{tabular}{llllll}
\hline \hline$\Delta_{1}$ & $\Delta_{2}$ & $\Delta_{3}$ & \multicolumn{1}{c}{$f_{1}$} & \multicolumn{1}{c}{$f_{2}$} & \multicolumn{1}{c}{$f_{3}$} \\
\hline$\pi / 2$ & $\pi / 2$ & $\pi / 2$ & \multicolumn{1}{c}{+3.0545} & -0.4999 & -3.0466 \\
$\pi / 4$ & $\pi / 2$ & $\pi / 4$ & $+4.2215-0.0491 \mathfrak{n}_{1}$ & $-0.4996+0.0000 \mathfrak{n}_{1}$ & $-4.1710-0.2943 \mathfrak{n}_{1}$ \\
& & & $-0.1473 \mathfrak{n}_{2}-0.0491 \mathfrak{n}_{3}$ & $+0.0000 \mathfrak{n}_{2}+0.0000 \mathfrak{n}_{3}$ & $+0.0645 \mathfrak{n}_{2}-0.2943 \mathfrak{n}_{3}$ \\
0.3 & 0.4 & 0.5 & $+7.9855-0.2597 \mathfrak{n}_{1}$ & $-0.4994-0.0061 \mathfrak{n}_{1}$ & $-9.8404-0.9312 \mathfrak{n}_{1}$ \\
& & & $-0.5833 \mathfrak{n}_{2}-0.6411 \mathfrak{n}_{3}$ & $-0.0020 \mathfrak{n}_{2}-0.0007 \mathfrak{n}_{3}$ & $-0.0293 \mathfrak{n}_{2}+0.3739 \mathfrak{n}_{3}$ \\
0.4 & 0.5 & 0.7 & $+6.6696-0.1904 \mathfrak{n}_{1}$ & $-0.4986-0.0016 \mathfrak{n}_{1}$ & $-7.5313-0.6893 \mathfrak{n}_{1}$ \\
& & & $-0.4166 \mathfrak{n}_{2}-0.4915 \mathfrak{n}_{3}$ & $-0.0008 \mathfrak{n}_{2}-0.0001 \mathfrak{n}_{3}$ & $-0.1581 \mathfrak{n}_{2}+0.2767 \mathfrak{n}_{3}$ \\
\hline \hline
\end{tabular}

\section{THE TOPOLOGICALLY TWISTED INDEX OF $N^{0,1,0}$}

In this section we study the Chern-Simons matter theory whose holographic dual is described by M-theory on $\mathrm{AdS}_{4} \times N^{0,1,0} / \mathbb{Z}_{k}$ [35-37]. The space $N^{0,1,0}$ is a homogeneous Sasaki-Einstein manifold of dimension seven and defined as the coset $S U(3) / U(1)$. The manifold has the isometry $S U(3) \times S U(2)$; the latter $S U(2)$ is identified with the R-symmetry.

The field theory was discussed in [38-40] and shown to be described by the following quiver diagram:

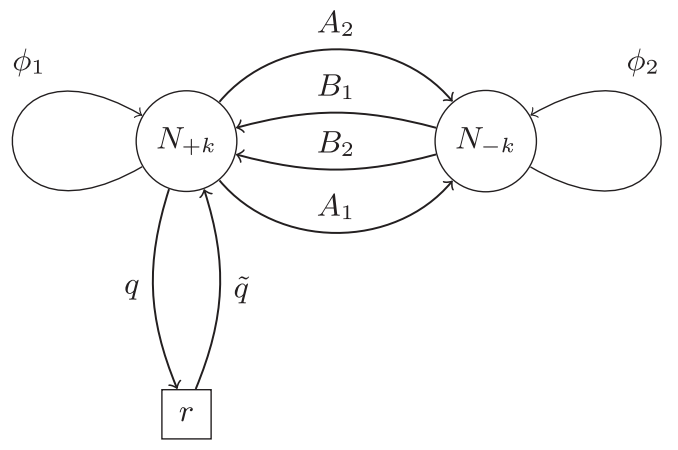

The superpotential is

$$
\begin{aligned}
W= & \operatorname{Tr}\left(A_{1} \phi_{2} B_{2}-B_{2} \phi_{1} A_{1}-A_{2} \phi_{2} B_{1}+B_{1} \phi_{1} A_{2}\right. \\
& \left.+\frac{k}{2} \phi_{1}^{2}-\frac{k}{2} \phi_{2}^{2}+\tilde{q} \phi_{1} q\right) .
\end{aligned}
$$

The free energy on $S^{3}$ has been shown to match the gravity computation [38]; a discussion of the superconformal index was presented in $[39,40]$. In the context of the topologically twisted index, this theory was recently considered by Hosseini and Mekareeya in [26] from which we borrow much, including the notation and the leading order analysis.

\section{A. Numerical solutions to the system of BAEs}

The topologically twisted index can be algorithmically assembled from the field theory content and the result is

$$
\begin{aligned}
Z= & \frac{1}{(N !)^{2}} \sum_{\mathfrak{m}, \tilde{\mathfrak{m}} \in \mathbb{Z}^{N}} \int_{\mathcal{C}} \prod_{i=1}^{N} \frac{d x_{i}}{2 \pi i x_{i}} \frac{d \tilde{x}_{i}}{2 \pi i \tilde{x}_{i}} x_{i}^{k \mathfrak{m}_{i}} \tilde{x}_{i}^{-k \tilde{\mathfrak{m}}_{i}} \times \prod_{i \neq j}^{N}\left(1-\frac{x_{i}}{x_{j}}\right)\left(1-\frac{\tilde{x}_{i}}{\tilde{x}_{j}}\right) \\
& \times \prod_{i, j=1}^{N} \prod_{a=1,2}\left(\frac{\sqrt{\frac{x_{i}}{\tilde{x}_{j}} y_{a}}}{1-\frac{x_{i}}{\tilde{x}_{j}} y_{a}}\right)^{\mathfrak{m}_{i}-\tilde{\mathfrak{m}}_{j}-\mathfrak{n}_{a}+1} \prod_{b=3,4}\left(\frac{\sqrt{\frac{\tilde{x}_{j}}{x_{i}} y_{b}}}{1-\frac{\tilde{x}_{j}}{x_{i}} y_{b}}\right)^{\tilde{\mathfrak{m}}_{j}-\mathfrak{m}_{i}-\mathfrak{n}_{b}+1} \\
& \times \prod_{i=1}^{N}\left(\frac{\sqrt{x_{i} y_{q}}}{1-x_{i} y_{q}}\right)^{r\left(\mathfrak{m}_{i}-\mathfrak{n}_{q}+1\right)}\left(\frac{\sqrt{\frac{1}{x_{i}} y_{\tilde{q}}}}{1-\frac{1}{x_{i}} y_{\tilde{q}}}\right)^{r\left(-\mathfrak{m}_{i}-\mathfrak{n}_{\tilde{q}}+1\right)}
\end{aligned}
$$


Performing the summation over magnetic fluxes by introducing a large cutoff $M$ we get

$$
\begin{aligned}
Z= & \frac{1}{(N !)^{2}} \int_{\mathcal{C}} \prod_{i=1}^{N} \frac{d x_{i}}{2 \pi i x_{i}} \frac{d \tilde{x}_{i}}{2 \pi i \tilde{x}_{i}} \prod_{i \neq j}^{N}\left(1-\frac{x_{i}}{x_{j}}\right)\left(1-\frac{\tilde{x}_{i}}{\tilde{x}_{j}}\right) \times \prod_{i, j=1}^{N} \prod_{a=1,2}\left(\frac{\sqrt{\frac{x_{i}}{\tilde{x}_{j}} y_{a}}}{1-\frac{x_{i}}{\tilde{x}_{j}} y_{a}}\right)^{1-\mathfrak{n}_{a}} \prod_{b=3,4}\left(\frac{\sqrt{\frac{\tilde{x}_{j}}{x_{i}} y_{b}}}{1-\frac{\tilde{x}_{j}}{x_{i}} y_{b}}\right)^{1-\mathfrak{n}_{b}} \\
& \times \prod_{i=1}^{N}\left(\frac{\sqrt{x_{i} y_{q}}}{1-x_{i} y_{q}}\right)^{r\left(1-\mathfrak{n}_{q}\right)}\left(\frac{\sqrt{\frac{1}{x_{i}} y_{\tilde{q}}}}{1-\frac{1}{x_{i}} y_{\tilde{q}}}\right)^{r\left(1-\mathfrak{n}_{\tilde{q}}\right)} \times \prod_{i=1}^{N} \frac{\left(e^{i B_{i}}\right)^{M}}{e^{i B_{i}}-1} \prod_{j=1}^{N} \frac{\left(e^{i \tilde{B}_{j}}\right)^{M}}{e^{i \tilde{B}_{j}}-1},
\end{aligned}
$$

where the Bethe Ansatz equations are

$$
\begin{aligned}
& 1=e^{i B_{i}}=x_{i}^{k} \prod_{j=1}^{N} \frac{\left(1-y_{3} \frac{\tilde{x}_{j}}{x_{i}}\right)\left(1-y_{4} \frac{\tilde{x}_{j}}{x_{i}}\right)}{\left(1-y_{1}^{-1} \frac{\tilde{x}_{j}}{x_{i}}\right)\left(1-y_{2}^{-1} \frac{\tilde{x}_{j}}{x_{i}}\right)} \times\left(\frac{\sqrt{x_{i} y_{q}}}{1-x_{i} y_{q}}\right)^{r}\left(\frac{\sqrt{\frac{1}{x_{i}} y_{\tilde{q}}}}{1-\frac{1}{x_{i}} y_{\tilde{q}}}\right)^{-r}, \\
& 1=e^{i \tilde{B}_{j}}=\tilde{x}_{j}^{k} \prod_{i=1}^{N} \frac{\left(1-y_{3} \frac{\tilde{x}_{j}}{x_{i}}\right)\left(1-y_{4} \frac{\tilde{x}_{j}}{x_{i}}\right)}{\left(1-y_{1}^{-1} \frac{\tilde{x}_{j}}{x_{i}}\right)\left(1-y_{2}^{-1} \frac{\tilde{x}_{j}}{x_{i}}\right)} .
\end{aligned}
$$

The compact expression for the index in terms of solutions to the Bethe-Ansatz equations Eq. (3.5) takes the form

$$
\begin{aligned}
Z\left(y_{a}, \mathfrak{n}_{a}\right)= & (-1)^{\frac{N r}{2}} y_{q}^{-\frac{1}{2} N r n_{q}} y_{\tilde{q}}^{-\frac{1}{2} N r n_{\tilde{q}}} \prod_{a=1}^{4} y_{a}^{-\frac{1}{2} N^{2} \mathfrak{n}_{a}} \times \sum_{I \in B A E}\left[\frac{1}{\operatorname{det} \mathbb{B}} \frac{\prod_{i=1}^{N} x_{i}^{N} \tilde{x}_{i}^{N} \prod_{i \neq j}\left(1-\frac{x_{i}}{x_{j}}\right)\left(1-\frac{\tilde{x}_{i}}{\tilde{x}_{j}}\right)}{\prod_{i, j=1}^{N} \prod_{a=1,2}\left(\tilde{x}_{j}-y_{a} x_{i}\right)^{1-\mathfrak{n}_{a}} \prod_{a=3,4}\left(x_{i}-y_{a} \tilde{x}_{j}\right)^{1-\mathfrak{n}_{a}}}\right. \\
& \left.\times \prod_{i=1}^{N} \frac{x_{i}^{\frac{1}{2} r}}{\left(1-x_{i} y_{q}\right)^{r\left(1-\mathfrak{n}_{q}\right)}\left(x_{i}-y_{\tilde{q}}\right)^{r\left(1-\mathfrak{n}_{\tilde{q}}\right)}}\right] .
\end{aligned}
$$

The transformation matrix $\mathbb{B}$ describing the change in integration variables from $x_{i}$ to $B_{i}$ is

$$
\left.\mathbb{B}\right|_{\mathrm{BAEs}}=\left(\begin{array}{cc}
\delta_{j l}\left[k-\sum_{m=1}^{N} G_{j m}+r x_{j}\left(\frac{1}{x_{j}-y_{\tilde{q}}}-\frac{1}{x_{j}-y_{q}^{-1}}\right)\right] & G_{j l} \\
-G_{l j} & \delta_{j l}\left[k+\sum_{m=1}^{N} G_{m j}\right]
\end{array}\right),
$$

where

$$
D(z)=\frac{\left(1-z y_{3}\right)\left(1-z y_{4}\right)}{\left(1-z y_{1}^{-1}\right)\left(1-z y_{2}^{-1}\right)}, \quad G_{i j}=\left.\frac{\partial \log D(z)}{\partial \log z}\right|_{z=\tilde{x}_{j} / x_{i}} .
$$

The Bethe-Ansatz equations Eq. (3.5) can be obtained from the potential which takes the form

$$
\begin{aligned}
\mathcal{V}= & \sum_{i=1}^{N}\left[\frac{k}{2}\left(\tilde{u}_{i}^{2}-u_{i}^{2}\right)-2 \pi\left(\tilde{n}_{i} \tilde{u}_{i}-n_{i} u_{i}\right)\right]+\sum_{i, j=1}^{N}\left[\sum_{a=3,4} \operatorname{Li}_{2}\left(e^{i\left(\tilde{u}_{j}-u_{i}+\Delta_{a}\right)}\right)-\sum_{a=1,2} \operatorname{Li}_{2}\left(e^{i\left(\tilde{u}_{j}-u_{i}-\Delta_{a}\right)}\right)\right] \\
& +r \sum_{i=1}^{N}\left[\operatorname{Li}_{2}\left(e^{i\left(-u_{i}+\Delta_{\tilde{q}}\right)}\right)-\operatorname{Li}_{2}\left(e^{i\left(-u_{i}-\Delta_{q}\right)}\right)\right]+\frac{r}{2} \sum_{i=1}^{N}\left[\left(\Delta_{\tilde{q}}+\Delta_{q}-2 \pi\right) u_{i}\right],
\end{aligned}
$$

where

$$
\sum_{i=1}^{N}\left[-2 \pi\left(\tilde{n}_{i} \tilde{u}_{i}-n_{i} u_{i}\right)\right]=\left(4 \pi-\sum_{a=1}^{4} \Delta_{a}\right) \sum_{i>j}^{N}\left(\tilde{u}_{j}-u_{i}\right)=2 \pi \sum_{i>j}^{N}\left(\tilde{u}_{j}-u_{i}\right) .
$$




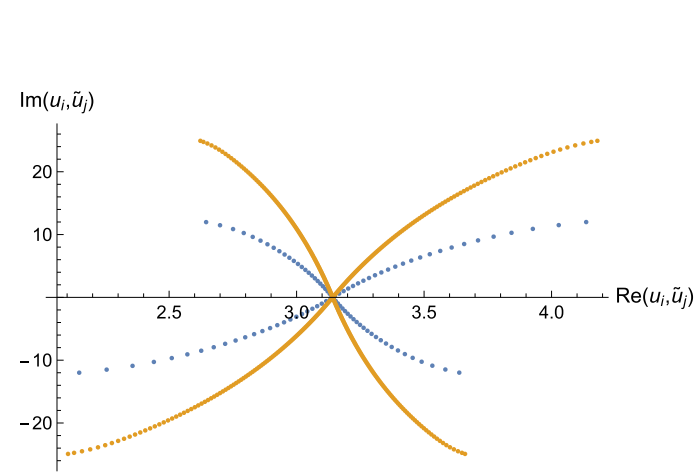

(a) Eigenvalue distribution

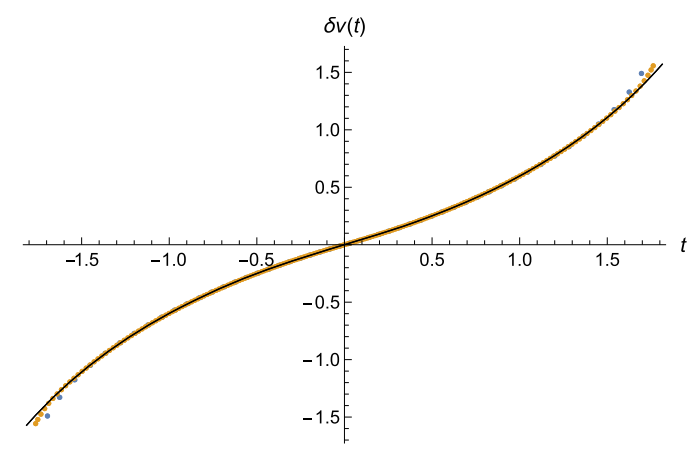

(c) Real part difference $\delta v(t)$

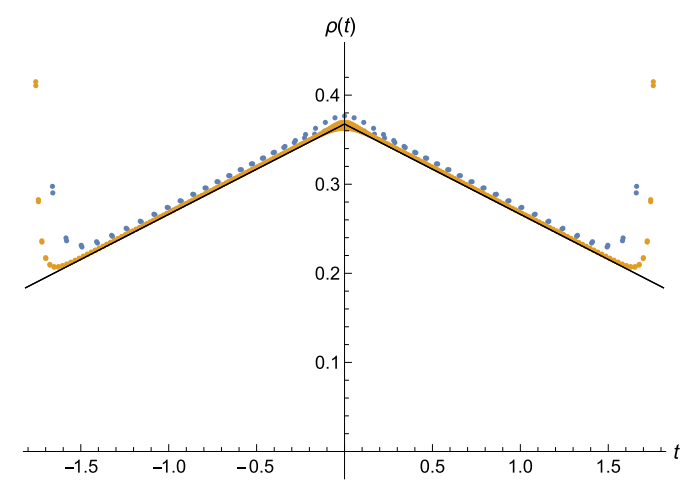

(b) Eigenvalue density $\rho(t)$

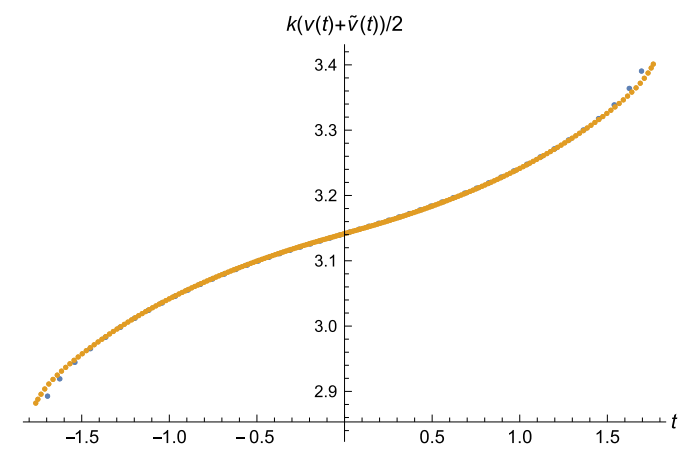

(d) Real part axis $k(v(t)+\tilde{v}(t)) / 2$

$$
\begin{aligned}
& N=50, k=1, \\
& \Delta_{a}=\left\{\frac{\pi}{2}, \frac{\pi}{2}, \frac{\pi}{2}, \frac{\pi}{2}\right\}, \\
& r=1, \\
& \left\{\Delta_{q}, \Delta_{\tilde{q}}\right\}=\left\{-\frac{\pi}{2}, \frac{3 \pi}{2}\right\} . \\
& N=200, k=1, \\
& \Delta_{a}=\left\{\frac{\pi}{2}, \frac{\pi}{2}, \frac{\pi}{2}, \frac{\pi}{2}\right\}, \\
& r=1, \\
& \left\{\Delta_{q}, \Delta_{\tilde{q}}\right\}=\left\{-\frac{\pi}{2}, \frac{3 \pi}{2}\right\} .
\end{aligned}
$$

FIG. 3. Eigenvalues for the special case $\Delta_{a}=\left\{\frac{\pi}{2}, \frac{\pi}{2}, \frac{\pi}{2}, \frac{\pi}{2}\right\}$ for $N=50$ (blue) and 200 (orange) with the same other parameters.

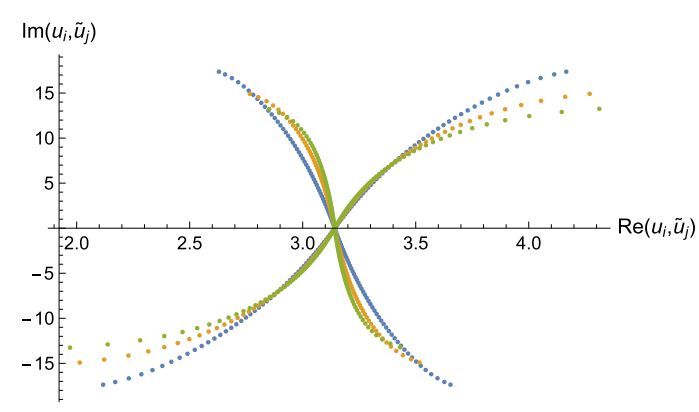

(a) Eigenvalue distribution

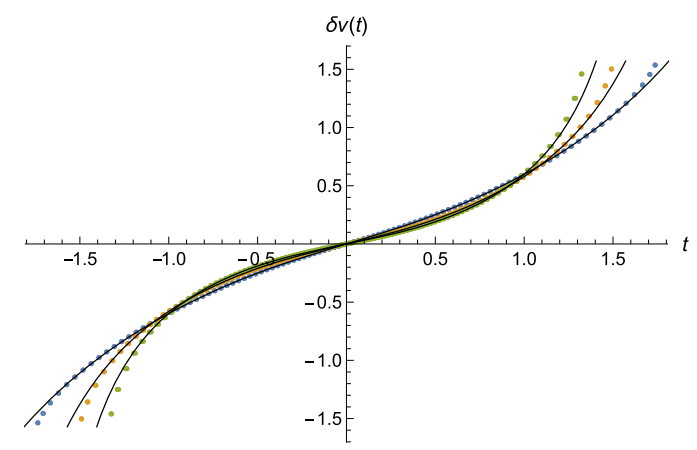

(c) Real part difference $\delta v(t)$

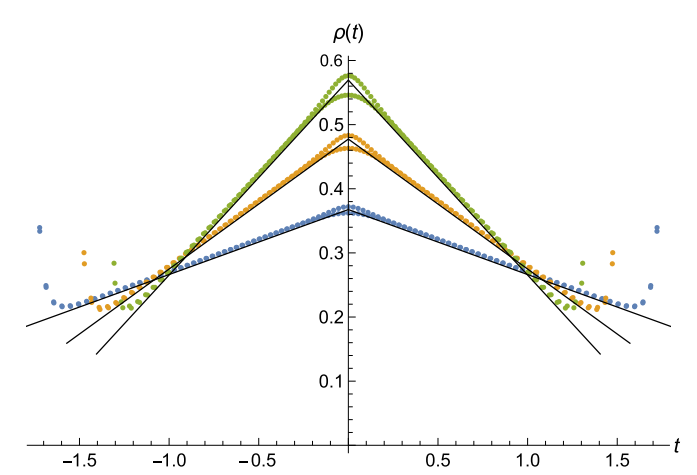

(b) Eigenvalue density $\rho(t)$

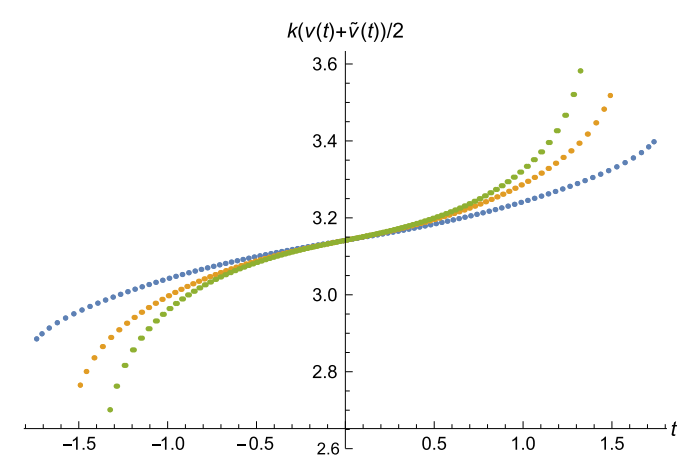

(d) Real part axis $k(v(t)+\tilde{v}(t)) / 2$
$N=100, k=1$,

$\Delta_{a}=\left\{\frac{\pi}{2}, \frac{\pi}{2}, \frac{\pi}{2}, \frac{\pi}{2}\right\}$,

$r=1$,

$\left\{\Delta_{q}, \Delta_{\tilde{q}}\right\}=\left\{-\frac{\pi}{2}, \frac{3 \pi}{2}\right\}$.

$N=100, k=1$,

$\Delta_{a}=\left\{\frac{\pi}{2}, \frac{\pi}{2}, \frac{\pi}{2}, \frac{\pi}{2}\right\}$,

$r=2$,

$\left\{\Delta_{q}, \Delta_{\tilde{q}}\right\}=\left\{-\frac{\pi}{2}, \frac{3 \pi}{2}\right\}$.

$N=100, k=1$,

$\Delta_{a}=\left\{\frac{\pi}{2}, \frac{\pi}{2}, \frac{\pi}{2}, \frac{\pi}{2}\right\}$,

$r=3$,

$\left\{\Delta_{q}, \Delta_{\tilde{q}}\right\}=\left\{-\frac{\pi}{2}, \frac{3 \pi}{2}\right\}$.

FIG. 4. Eigenvalues for the special case $\Delta_{a}=\left\{\frac{\pi}{2}, \frac{\pi}{2}, \frac{\pi}{2}, \frac{\pi}{2}\right\}$ for $r=1$ (blue), 2 (orange) and 3 (green) with other parameters kept the same. 
We use the leading order solution to the system of BAEs in [26] as a seed for the exact numerical solution to the BAE's in Eq. (3.5). We will assume, as in [25], that $0<$ $v(t)+\Delta_{q}<2 \pi$ and $0<-v(t)+\Delta_{\tilde{q}}<2 \pi$, then we have

$$
-\Delta_{q}<v(t)<\Delta_{\tilde{q}},
$$

thus we set the initial real part axis to be

$$
\frac{v(t)+\tilde{v}(t)}{2}=\frac{\Delta_{\tilde{q}}-\Delta_{q}}{2} .
$$

The marginality condition on the superpotential requires that $\Delta_{q}+\Delta_{\tilde{q}}=\pi$. For comparison, recall that in the ABJM theory, the real part axis defined above is $\pi$ when $k=1$, thus we assume the range of the real part axis here should be near to $\pi$ to match with the ABJM theory when $r=0$. If we set $\left(\Delta_{\tilde{q}}-\Delta_{q}\right) / 2=\pi$ we will get $\left\{\Delta_{q}, \Delta_{\tilde{q}}\right\}=$ $\{-\pi / 2,3 \pi / 2\}$. We will see the effects of the values of $\left\{\Delta_{q}, \Delta_{\tilde{q}}\right\}$ in the numerical solutions.

Before entering the details of the numerical analysis, let us remark that, as compared to ABJM, the system has three new parameters which are $r$-the number of flavors of fundamental hypermultiplets, and the fugacities $\left\{\Delta_{q}, \Delta_{\tilde{q}}\right\}$ discussed above. Our goal is to explore the space of new parameters as well as those parameters already present in
ABJM, namely $N$-the rank of the gauge group and $\Delta_{a}=\left\{\Delta_{1}, \Delta_{2}, \Delta_{3}, \Delta_{4}\right\}$-the fugacities of the bifundamental matter.

The numerical solutions to the BAEs can be obtained using FindRoot in Mathematica as implemented in [20,41]. In the following we focus on the case $k=1$. The numerical solutions for different values of $N, r,\left\{\Delta_{q}, \Delta_{\tilde{q}}\right\}$ and $\Delta_{a}$ are shown in Figs. 3-6. The black lines are the analytical results in [26].

The numerical solutions show that the eigenvalues are not reflectively symmetric about $\pi$ alone the real axis as the ABJM theory. Furthermore, the imaginary part of $u_{i}$ is not exactly the same as $\tilde{u}_{i}$ so that there are two numerical results of the eigenvalue density $\rho(t)$, the real part difference $\delta v(t)$ and the real part axis $(v(t)+\tilde{v}(t)) / 2$, though it is not obvious in the last two because of overlapping.

In Fig. 3 we describe the eigenvalues as exact numerical solutions of the BAE Eq. (3.5). The plots show that the imaginary part of the eigenvalues scales as $N^{1 / 2}$. The eigenvalue densities are very well described by the leading analytical result of [26] except for some deviations at the edges of the intervals.

Figure 4 explores the nature of the eigenvalues as one changes the number of fundamental flavors $r$, the most prominent change is accurately captured by the slope in the eigenvalue density. In Fig. 5 we explore the effects of

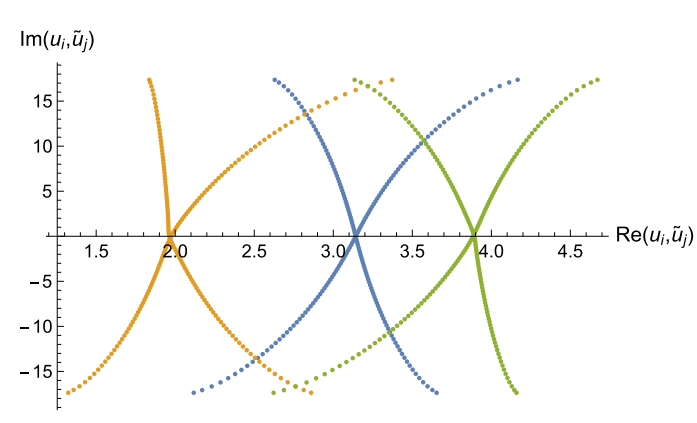

(a) Eigenvalue distribution

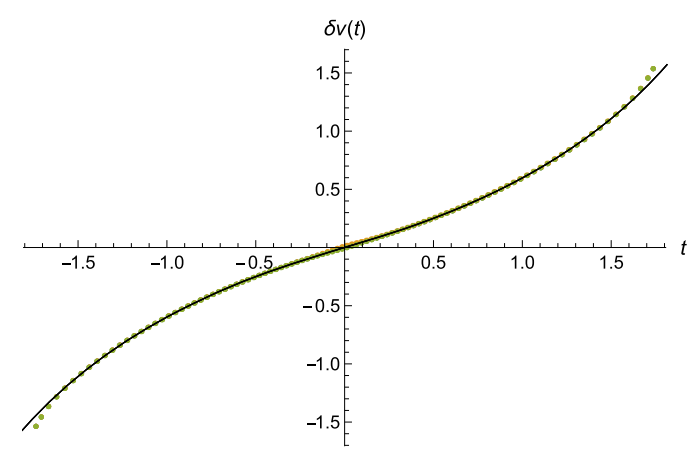

(c) Real part difference $\delta v(t)$

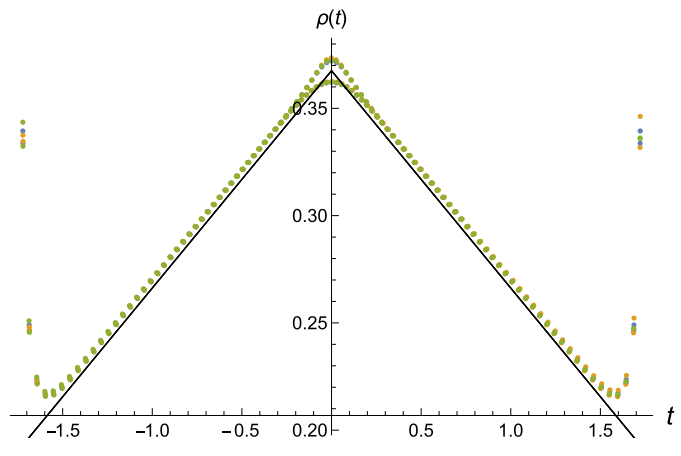

(b) Eigenvalue density $\rho(t)$

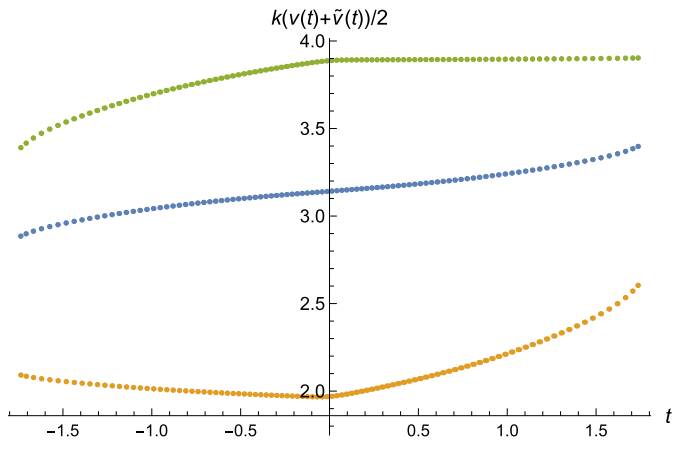

(d) Real part axis $k(v(t)+\tilde{v}(t)) / 2$

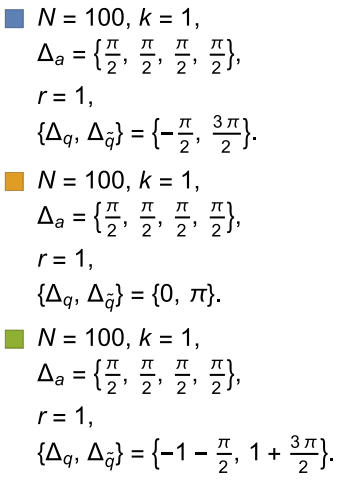

FIG. 5. Eigenvalues for the special case $\Delta_{a}=\left\{\frac{\pi}{2}, \frac{\pi}{2}, \frac{\pi}{2}, \frac{\pi}{2}\right\}$ for $\left\{\Delta_{q}, \Delta_{\tilde{q}}\right\}=\left\{-\frac{\pi}{2}, \frac{3 \pi}{2}\right\}$ (blue), $\{0, \pi\}$ (orange) and $\left\{-1-\frac{\pi}{2}, 1+\frac{3 \pi}{2}\right\}$ (green) with the same other parameters. 


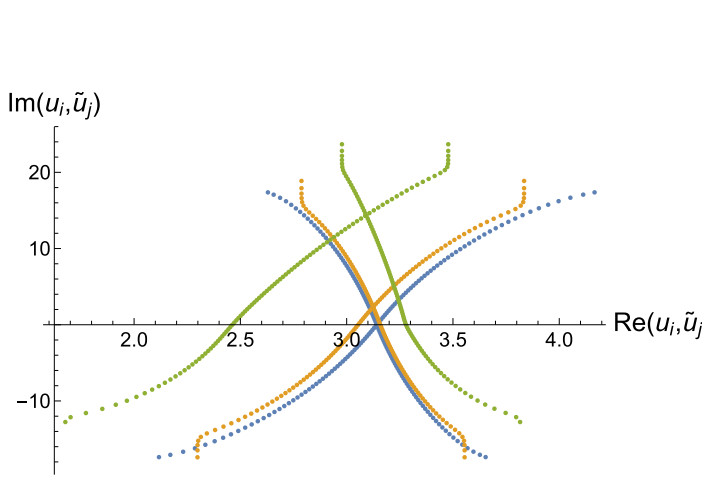

(a) Eigenvalue distribution

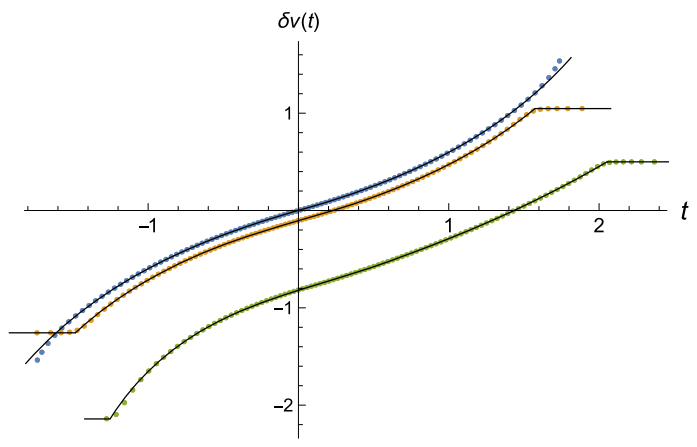

(c) Real part difference $\delta v(t)$

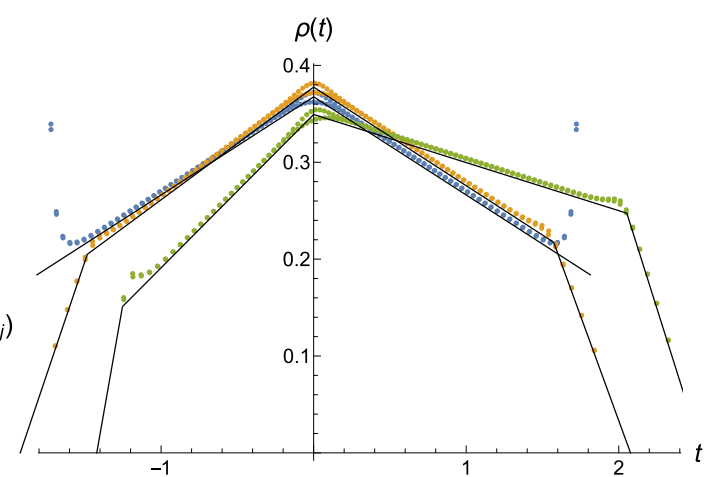

(b) Eigenvalue density $\rho(t)$

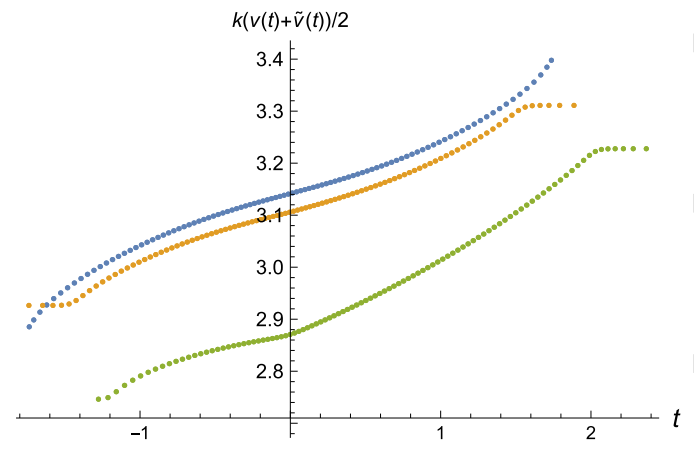

(d) Real part axis $k(v(t)+\tilde{v}(t)) / 2$

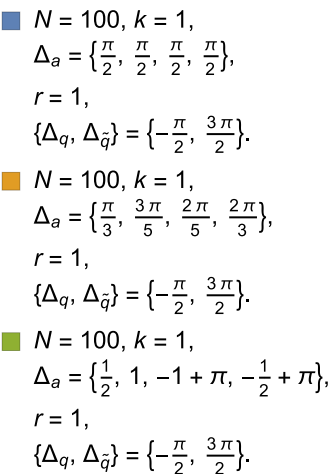

FIG. 6. Eigenvalues for the special case $\Delta_{a}=\left\{\frac{\pi}{2}, \frac{\pi}{2}, \frac{\pi}{2}, \frac{\pi}{2}\right\}$ (blue), and the general cases $\Delta_{a}=\left\{\frac{\pi}{3}, \frac{3 \pi}{5}, \frac{2 \pi}{5}, \frac{2 \pi}{3}\right\}$ (orange) and $\Delta_{a}=$ $\left\{\frac{1}{2}, 1,-1+\pi,-\frac{1}{2}+\pi\right\}$ (green) with the same other parameters.

changing the fugacities $\left\{\Delta_{q}, \Delta_{\tilde{q}}\right\}$. The main effect is in the real part axis $(v(t)+\tilde{v}(t)) / 2$.

Finally, in Fig. 6 we explore the eigenvalues away from the symmetric point of the bifundamental fugacities. As in the ABJM case [20], this is numerically challenging as one needs confront numerically, various numerical singularities due to branch points in the polylogarithmic functions.

\section{B. The subleading term of the index at large $N$}

Having achieved control of the eigenvalues we can proceed to analyze the index. As the ABJM theory, we expand the index beyond the leading order in $N$ and we expect the subleading behavior of the index to have the form

$$
\begin{aligned}
\operatorname{Re} \log Z= & f_{1}(k, r, \Delta, \mathfrak{n}) N^{3 / 2}+f_{2}(k, r, \Delta, \mathfrak{n}) N^{1 / 2} \\
& +f_{3}(k, r, \Delta, \mathfrak{n}) \log N+f_{4}(k, r, \Delta, \mathfrak{n}) \\
& +\mathcal{O}\left(N^{-1 / 2}\right),
\end{aligned}
$$

where the functions $f_{1}, f_{2}, f_{3}$ and $f_{4}$ are linear in the magnetic fluxes $\mathfrak{n}$.

For $k=1$ and a given set of $\Delta_{a}, r$ and $\left\{\Delta_{q}, \Delta_{\tilde{q}}\right\}$, we can compute the index Eq. (3.6) and $\operatorname{Re} \log Z$ for a range of $N$ using the numerical solutions obtained in Sec. III A. Then we decompose $\operatorname{Re} \log Z$ into a sum of four independent terms

$$
\operatorname{Re} \log Z=A+B_{1} \mathfrak{n}_{3}+B_{2} \mathfrak{n}_{4}+B_{3} \mathfrak{n}_{q}
$$

where we have used the marginality condition on the superpotential $\mathfrak{n}_{1}+\mathfrak{n}_{4}=1, \mathfrak{n}_{2}+\mathfrak{n}_{3}=1$ and $\mathfrak{n}_{q}+\mathfrak{n}_{\tilde{q}}=1$. Then we perform a linear least-squares fit for $A$ and $B_{a}$ to the function

$$
\begin{aligned}
f(N)= & f_{1} N^{3 / 2}+f_{2} N^{1 / 2}+f_{3} \log N+f_{4} \\
& +\sum_{p=1}^{p_{c}} f_{p+4} N^{(1-2 p) / 2},
\end{aligned}
$$

where $p_{c}$ is the cutoff needed for the numerical fitting. Notice that the inverse powers of $N$ should be $N^{(1-2 p) / 2}$, instead of $N^{p / 2}$, for a integer $p$ as before, because of the stability which has been checked numerically.

The results of the numerical fit for $\operatorname{Re} \log Z$ with $N$ are presented in Table II. The error between the analytical leading term computed by the index theorem in [26] and the numerical leading term $f_{1} N^{3 / 2}$ is negligible. More 
TABLE II. $\quad\left(N^{0,1,0}\right)$ Numerical fit for Re $\log Z=f_{1} N^{3 / 2}+f_{2} N^{1 / 2}+f_{3} \log N+f_{4}+\sum_{p=1}^{p_{c}=5} N^{(1-2 p) / 2}$. The $s$ in the bracket of $N$ is the step of $N$.

\begin{tabular}{|c|c|c|c|c|c|c|c|}
\hline \multicolumn{8}{|c|}{ (a) $k=1 ;\left\{\Delta_{1}, \Delta_{2}, \Delta_{3}, \Delta_{4}\right\}=\left\{\frac{\pi}{2}, \frac{\pi}{2}, \frac{\pi}{2}, \frac{\pi}{2}\right\}$} \\
\hline$r$ & $\Delta_{q}$ & $\Delta_{\tilde{q}}$ & $N(s)$ & $f_{1}$ & $f_{2}$ & $f_{3}$ & $f_{4}$ \\
\hline \multirow[t]{3}{*}{1} & $-\frac{\pi}{2}$ & $\frac{3 \pi}{2}$ & $100 \sim 300(10)$ & -2.41840 & +2.11612 & -0.50066 & -2.29495 \\
\hline & 0 & $\pi$ & $100 \sim 300(10)$ & -2.41840 & $\begin{array}{l}+1.73825 \\
+0.15115 \mathfrak{n}_{3} \\
+0.15115 \mathfrak{n}_{4} \\
+0.45345 \mathfrak{n}_{q}\end{array}$ & -0.50056 & -2.29565 \\
\hline & $-\frac{\pi}{2}-1$ & $\frac{3 \pi}{2}+1$ & $100 \sim 300(10)$ & -2.41840 & $\begin{array}{l}+2.35669 \\
-0.09623 \mathfrak{n}_{3} \\
-0.09623 \mathfrak{n}_{4} \\
-0.28868 \mathfrak{n}_{q}\end{array}$ & -0.50080 & -2.29412 \\
\hline 2 & $-\frac{\pi}{2}$ & $\frac{3 \pi}{2}$ & $100 \sim 300(10)$ & -3.14159 & +2.08351 & -0.50065 & -2.49377 \\
\hline 3 & $-\frac{\pi}{2}$ & $\frac{3 \pi}{2}$ & $100 \sim 300(10)$ & -3.74657 & +2.38846 & -0.50068 & -3.10991 \\
\hline
\end{tabular}

(b) $k=1 ;\left\{\Delta_{1}, \Delta_{2}, \Delta_{3}, \Delta_{4}\right\}=\left\{\frac{\pi}{3}, \frac{3 \pi}{5}, \frac{2 \pi}{5}, \frac{2 \pi}{3}\right\}$

\begin{tabular}{|c|c|c|c|c|c|c|c|}
\hline$r$ & $\Delta_{q}$ & $\Delta_{\tilde{q}}$ & $N(s)$ & $f_{1}$ & $f_{2}$ & $f_{3}$ & $f_{4}$ \\
\hline \multirow[t]{3}{*}{1} & $-\frac{\pi}{2}$ & $\frac{3 \pi}{2}$ & $100 \sim 200(5)$ & $\begin{array}{l}-2.45347 \\
-0.37599 \mathfrak{n}_{3} \\
+0.44419 \mathfrak{n}_{4}\end{array}$ & $\begin{array}{l}+2.16951 \\
-0.00208 \mathfrak{n}_{3} \\
+0.01685 \mathfrak{n}_{4} \\
+0.01959 \mathfrak{n}_{q}\end{array}$ & $\begin{array}{l}-0.50086 \\
-0.00002 \mathfrak{n}_{3} \\
+0.00002 \mathfrak{n}_{4}\end{array}$ & $\begin{array}{l}-2.37003 \\
-0.00489 \mathfrak{n}_{3} \\
+0.00569 \mathfrak{n}_{4}\end{array}$ \\
\hline & 0 & $\pi$ & $85 \sim 105(1)$ & $\begin{array}{l}-2.45347 \\
-0.37599 \mathfrak{n}_{3} \\
+0.44419 \mathfrak{n}_{4}\end{array}$ & $\begin{array}{l}+1.81164 \\
+0.16898 \mathfrak{n}_{3} \\
+0.13521 \mathfrak{n}_{4} \\
+0.46029 \mathfrak{n}_{q}\end{array}$ & $\begin{array}{l}-0.50107 \\
-0.00007 \mathfrak{n}_{3} \\
+0.00008 \mathfrak{n}_{4} \\
-0.00002 \mathfrak{n}_{q}\end{array}$ & $\begin{array}{l}-2.36898 \\
-0.00460 \mathfrak{n}_{3} \\
+0.00537 \mathfrak{n}_{4} \\
+0.00012 \mathfrak{n}_{q}\end{array}$ \\
\hline & $-\frac{\pi}{2}-1$ & $\frac{3 \pi}{2}+1$ & $100 \sim 200(5)$ & $\begin{array}{l}-2.45347 \\
-0.37599 \mathfrak{n}_{3} \\
+0.44419 \mathfrak{n}_{4}\end{array}$ & $\begin{array}{l}+2.40631 \\
-0.08786 \mathfrak{n}_{3} \\
-0.08581 \mathfrak{n}_{4} \\
-0.26097 \mathfrak{n}_{q}\end{array}$ & $\begin{array}{l}-0.50082 \\
-0.00001 \mathfrak{n}_{3} \\
+0.00003 \mathfrak{n}_{4}\end{array}$ & $\begin{array}{l}-2.37028 \\
-0.00493 \mathfrak{n}_{3} \\
+0.00566 \mathfrak{n}_{4}\end{array}$ \\
\hline 2 & $-\frac{\pi}{2}$ & $\frac{3 \pi}{2}$ & $90 \sim 110(1)$ & $\begin{array}{l}-3.16053 \\
-0.46062 \mathfrak{n}_{3} \\
+0.49512 \mathfrak{n}_{4}\end{array}$ & $\begin{array}{l}+2.13744 \\
+0.19592 \mathfrak{n}_{3} \\
-0.16992 \mathfrak{n}_{4} \\
+0.02533 \mathfrak{n}_{q}\end{array}$ & $\begin{array}{l}-0.50098 \\
+0.00024 \mathfrak{n}_{3} \\
-0.00043 \mathfrak{n}_{4} \\
-0.00007 \mathfrak{n}_{q}\end{array}$ & $\begin{array}{l}-2.59551 \\
-0.12623 \mathfrak{n}_{3} \\
+0.12251 \mathfrak{n}_{4} \\
+0.00039 \mathfrak{n}_{q}\end{array}$ \\
\hline 3 & $-\frac{\pi}{2}$ & $\frac{3 \pi}{2}$ & $80 \sim 100(1)$ & $\begin{array}{l}-3.75949 \\
-0.53656 \mathfrak{n}_{3} \\
+0.55794 \mathfrak{n}_{4}\end{array}$ & $\begin{array}{l}+2.47949 \\
+0.46309 \mathfrak{n}_{3} \\
-0.43345 \mathfrak{n}_{4} \\
+0.02703 \mathfrak{n}_{q}\end{array}$ & $\begin{array}{l}-0.50119 \\
+0.00691 \mathfrak{n}_{3} \\
-0.00646 \mathfrak{n}_{4} \\
+0.00146 \mathfrak{n}_{q}\end{array}$ & $\begin{array}{l}-3.29270 \\
-0.37592 \mathfrak{n}_{3} \\
+0.36784 \mathfrak{n}_{4} \\
-0.00802 \mathfrak{n}_{q}\end{array}$ \\
\hline
\end{tabular}

\begin{tabular}{|c|c|c|c|c|c|c|c|}
\hline \multicolumn{8}{|c|}{ (c) $k=1 ;\left\{\Delta_{1}, \Delta_{2}, \Delta_{3}, \Delta_{4}\right\}=\left\{\frac{1}{2}, 1, \pi-1, \pi-\frac{1}{2}\right\}$} \\
\hline$r$ & $\Delta_{q}$ & $\Delta_{\tilde{q}}$ & $N(s)$ & $f_{1}$ & $f_{2}$ & $f_{3}$ & $f_{4}$ \\
\hline \multirow[t]{3}{*}{1} & $-\frac{\pi}{2}$ & $\frac{3 \pi}{2}$ & $100 \sim 140(2)$ & $\begin{array}{c}-2.69204 \\
+0.02322 \mathfrak{n}_{3} \\
+0.51870 \mathfrak{n}_{4}\end{array}$ & $\begin{array}{r}+2.10645 \\
+0.05070 \mathfrak{n}_{3} \\
+0.05726 \mathfrak{n}_{4} \\
+0.15070 \mathfrak{n}_{q}\end{array}$ & $\begin{array}{c}-0.50101 \\
-0.00001 \mathfrak{n}_{3}\end{array}$ & $\begin{array}{c}-2.42730 \\
+0.00051 \mathfrak{n}_{3} \\
+0.01181 \mathfrak{n}_{4} \\
+0.00005 \mathfrak{n}_{q}\end{array}$ \\
\hline & 0 & $\pi$ & $100 \sim 120(1)$ & $\begin{array}{c}-2.69204 \\
+0.02322 \mathfrak{n}_{3} \\
+0.51870 \mathfrak{n}_{4}\end{array}$ & $\begin{array}{l}+1.79254 \\
+0.19251 \mathfrak{n}_{3} \\
+0.14797 \mathfrak{n}_{4} \\
+0.58329 \mathfrak{n}_{q}\end{array}$ & $\begin{array}{c}-0.50079 \\
-0.00008 \mathfrak{n}_{3} \\
-0.00002 \mathfrak{n}_{q}\end{array}$ & $\begin{array}{r}-2.42886 \\
+0.00089 \mathfrak{n}_{3} \\
+0.01185 \mathfrak{n}_{4} \\
+0.00010 \mathfrak{n}_{q}\end{array}$ \\
\hline & $-\frac{\pi}{2}-1$ & $\frac{3 \pi}{2}+1$ & $100 \sim 120(1)$ & $\begin{array}{c}-2.69204 \\
+0.02322 \mathfrak{n}_{3} \\
+0.51870 \mathfrak{n}_{4}\end{array}$ & $\begin{array}{c}+2.33490 \\
-0.04105 \mathfrak{n}_{3} \\
-0.03333 \mathfrak{n}_{4} \\
-0.12470 \mathfrak{n}_{q}\end{array}$ & $\begin{array}{c}-0.50108 \\
+0.00002 \mathfrak{n}_{3} \\
-0.00005 \mathfrak{n}_{4}\end{array}$ & $\begin{array}{r}-2.42691 \\
+0.00031 \mathfrak{n}_{3} \\
+0.01213 \mathfrak{n}_{4} \\
-0.00003 \mathfrak{n}_{q}\end{array}$ \\
\hline
\end{tabular}


TABLE II. (Continued)

\begin{tabular}{|c|c|c|c|c|c|c|c|}
\hline \multicolumn{8}{|c|}{ (c) $k=1 ;\left\{\Delta_{1}, \Delta_{2}, \Delta_{3}, \Delta_{4}\right\}=\left\{\frac{1}{2}, 1, \pi-1, \pi-\frac{1}{2}\right\}$} \\
\hline$r$ & $\Delta_{q}$ & $\Delta_{\tilde{q}}$ & $N(s)$ & $f_{1}$ & $f_{2}$ & $f_{3}$ & $f_{4}$ \\
\hline 2 & $-\frac{\pi}{2}$ & $\frac{3 \pi}{2}$ & $80 \sim 180(5)$ & $\begin{array}{c}-3.28009 \\
-0.14052 \mathfrak{n}_{3} \\
+0.41670 \mathfrak{n}_{4}\end{array}$ & $\begin{array}{c}+1.91465 \\
+0.19945 \mathfrak{n}_{3} \\
-0.00011 \mathfrak{n}_{4} \\
+0.20126 \mathfrak{n}_{q}\end{array}$ & $\begin{array}{c}-0.50031 \\
-0.00026 \mathfrak{n}_{3} \\
-0.00042 \mathfrak{n}_{4} \\
-0.00040 \mathfrak{n}_{q}\end{array}$ & $\begin{array}{c}-2.52837 \\
-0.07857 \mathfrak{n}_{3} \\
+0.04734 \mathfrak{n}_{4} \\
+0.00239 \mathfrak{n}_{q}\end{array}$ \\
\hline 3 & $-\frac{\pi}{2}$ & $\frac{3 \pi}{2}$ & $100 \sim 140(2)$ & $\begin{array}{c}-3.83240 \\
-0.23232 \mathfrak{n}_{3} \\
+0.40375 \mathfrak{n}_{4}\end{array}$ & $\begin{array}{c}+2.18910 \\
+0.36535 \mathfrak{n}_{3} \\
-0.13462 \mathfrak{n}_{4} \\
+0.21699 \mathfrak{n}_{q}\end{array}$ & $\begin{aligned} & -0.50565{ }^{q} \\
+ & 0.00191 \mathfrak{n}_{3} \\
+ & 0.00215 \mathfrak{n}_{4} \\
+ & 0.00343 \mathfrak{n}_{q}\end{aligned}$ & $\begin{array}{r}-3.11766 \\
-0.20891 \mathfrak{n}_{3} \\
+0.14117 \mathfrak{n}_{4} \\
-0.01975 \mathfrak{n}_{q}\end{array}$ \\
\hline
\end{tabular}

precisely, the analytic leading order and the numerical result match to number of significant digits present in the table. The leading term is, indeed, independent of $\left\{\Delta_{q}, \Delta_{\tilde{q}}\right\}$. The numerical results indicate that the coefficient $f_{3}$ of the $\log N$ term is precisely $-1 / 2$.

The main result of this section is the numerical evidence pointing to the presence of a correction of the form $-\frac{1}{2} \log N$ in the topologically twisted index.

\section{THE TOPOLOGICALLY TWISTED INDEX OF $V^{5,2}$}

One particularly interesting model is the field theory dual to $\mathrm{AdS}_{4} \times V^{5,2} / \mathbb{Z}_{k}$ because the manifold $V^{5,2}$ is nontoric. A simple way to visualize this seven-dimensional manifold is as a homogeneous space $V^{5,2}=S O(5) / S O(3)$. There are two models for the dual field theory. Following the literature, we call model I, the proposal of Martelli and Sparks [42] and model II the proposal of Jafferis [43]. The free energy on $S^{3}$ of the field theories was discussed in [44-46] and perfect agreement at leading order was found with the dual supergravity solutions. The topologically twisted index for both models was studied at leading large $N$ order in [26] where the authors established the equivalence of both models. Here we go beyond the leading order in $N$ and demonstrate the equivalence of both models at the level of the topologically twisted index up to, and including, logarithmic in $N$ terms.

\section{A. Model I}

Model I was originally proposed in [42] with the following quiver diagram:

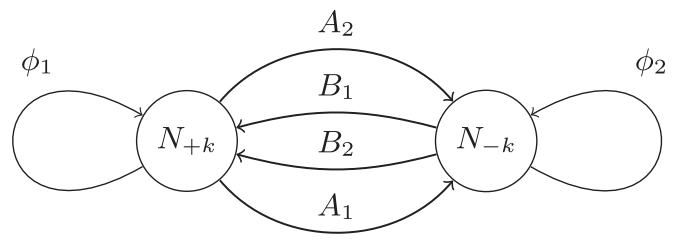

The superpotential accompanying the quiver diagram is

$$
W=\operatorname{Tr}\left[\phi_{1}^{3}+\phi_{2}^{3}+\phi_{1}\left(A_{1} B_{2}+A_{2} B_{1}\right)+\phi_{2}\left(B_{2} A_{1}+B_{1} A_{2}\right)\right]
$$

\section{Numerical solutions to the system of BAEs}

Collecting all the relevant building blocks following from the quiver diagram we have

$$
\begin{aligned}
& Z=\frac{1}{(N !)^{2}} \sum_{\mathfrak{m}, \tilde{\mathfrak{m}} \in \mathbb{Z}^{N}} \int_{\mathcal{C}} \prod_{i=1}^{N} \frac{d x_{i}}{2 \pi i x_{i}} \frac{d \tilde{x}_{i}}{2 \pi i \tilde{x}_{i}} x_{i}^{k \mathfrak{m}_{i}} \tilde{x}_{i}^{-k \tilde{\mathfrak{m}}_{i}} \times \prod_{i \neq j}^{N}\left(1-\frac{x_{i}}{x_{j}}\right)\left(1-\frac{\tilde{x}_{i}}{\tilde{x}_{j}}\right) \\
& \times \prod_{i, j=1}^{N}\left[\prod_{a=1,2}\left(\frac{\sqrt{\frac{x_{i}}{\tilde{x}_{j}} y_{a}}}{1-\frac{x_{i}}{\tilde{x}_{j}} y_{a}}\right)^{\mathfrak{m}_{i}-\tilde{\mathfrak{m}}_{j}-\mathfrak{n}_{a}+1} \prod_{b=3,4}\left(\frac{\sqrt{\frac{\tilde{x}_{j}}{x_{i}} y_{b}}}{1-\frac{\tilde{x}_{j}}{x_{i}} y_{b}}\right)\right. \\
& \left.\times\left(\frac{\sqrt{\frac{x_{i}}{x_{j}} y_{\phi_{1}}}}{1-\frac{x_{i}}{x_{j}} y_{\phi_{1}}}\right)^{\mathfrak{m}_{i}-\frac{1}{2} \mathfrak{n}_{\phi_{1}}+\frac{1}{2}}\left(\frac{\sqrt{\frac{x_{j}}{x_{i}} y_{\phi_{1}}}}{1-\frac{x_{j}}{x_{i}} y_{\phi_{1}}}\right)^{-\mathfrak{m}_{i}-\frac{1}{2} \mathfrak{n}_{\phi_{1}}+\frac{1}{2}} \times\left(\frac{\sqrt{\frac{\tilde{x}_{i}}{\tilde{x}_{j}} y_{\phi_{2}}}}{1-\frac{\tilde{x}_{i}}{\tilde{x}_{j}} y_{\phi_{2}}}\right)^{-\tilde{\mathfrak{m}}_{j}-\frac{1}{2} \mathfrak{n}_{\phi_{2}}+\frac{1}{2}}\left(\frac{\sqrt{\frac{\tilde{x}_{j}}{\tilde{x}_{i}} y_{\phi_{2}}}}{1-\frac{\tilde{x}_{j}}{\tilde{x}_{i}} y_{\phi_{2}}}\right)^{\tilde{\mathfrak{m}}_{j}-\frac{1}{2} \mathfrak{n}_{\phi_{2}}+\frac{1}{2}}\right] .
\end{aligned}
$$


Performing the summation over magnetic fluxes, as in previous cases, by introducing a large cutoff $M$ we get

$$
\begin{aligned}
Z= & \frac{1}{(N !)^{2}} \int_{\mathcal{C}} \prod_{i=1}^{N} \frac{d x_{i}}{2 \pi i x_{i}} \frac{d \tilde{x}_{i}}{2 \pi i \tilde{x}_{i}} \prod_{i \neq j}^{N}\left(1-\frac{x_{i}}{x_{j}}\right)\left(1-\frac{\tilde{x}_{i}}{\tilde{x}_{j}}\right) \\
& \times \prod_{i, j=1}^{N}\left[\prod_{a=1,2}\left(\frac{\sqrt{\frac{x_{i}}{\tilde{x}_{j}} y_{a}}}{1-\frac{x_{i}}{\tilde{x}_{j}} y_{a}}\right)^{1-\mathfrak{n}_{a}} \prod_{b=3,4}\left(\frac{\sqrt{\frac{\tilde{x}_{j}}{x_{i}} y_{b}}}{1-\frac{\tilde{x}_{j}}{x_{i}} y_{b}}\right)^{1-\mathfrak{n}_{b}}\right. \\
& \left.\times\left(\frac{\sqrt{y_{\phi_{1}}}}{1-\frac{x_{j}}{x_{i}} y_{\phi_{1}}}\right)^{1-\mathfrak{n}_{\phi_{1}}}\left(\frac{\sqrt{y_{\phi_{2}}}}{1-\frac{\tilde{x}_{i}}{\tilde{x}_{j}} y_{\phi_{2}}}\right)^{1-\mathfrak{n}_{\phi_{2}}}\right] \times \prod_{i=1}^{N} \frac{\left(e^{i B_{i}}\right)^{M}}{e^{i B_{i}}-1} \prod_{j=1}^{N} \frac{\left(e^{i \tilde{B}_{j}}\right)^{M}}{e^{i \tilde{B}_{j}}-1},
\end{aligned}
$$

where the Bethe Ansatz equations are

$$
\begin{aligned}
& 1=e^{i B_{i}}=x_{i}^{k} \prod_{j=1}^{N} \frac{\left(1-y_{3} \frac{\tilde{x}_{j}}{x_{i}}\right)\left(1-y_{4} \frac{\tilde{x}_{j}}{x_{i}}\right)}{\left(1-y_{1}^{-1} \frac{\tilde{x}_{j}}{x_{i}}\right)\left(1-y_{2}^{-1} \frac{\tilde{x}_{j}}{x_{i}}\right)}\left(\frac{1}{\sqrt{y_{1} y_{2} y_{3} y_{4}}}\right)\left(\frac{x_{i}-x_{j} y_{\phi_{1}}}{x_{j}-x_{i} y_{\phi_{1}}}\right), \\
& 1=e^{i \tilde{B}_{j}}=\tilde{x}_{j}^{k} \prod_{i=1}^{N} \frac{\left(1-y_{3} \frac{\tilde{x}_{j}}{x_{i}}\right)\left(1-y_{4} \frac{\tilde{x}_{j}}{x_{i}}\right)}{\left(1-y_{1}^{-1} \frac{\tilde{x}_{j}}{x_{i}}\right)\left(1-y_{2}^{-1} \frac{1}{\tilde{x}_{j}}\right)}\left(\frac{1}{\sqrt{y_{1} y_{2} y_{3} y_{4}}}\right)\left(\frac{\tilde{x}_{i}-\tilde{x}_{j} y_{\phi_{2}}}{\tilde{x}_{j}-\tilde{x}_{i} y_{\phi_{2}}}\right) .
\end{aligned}
$$

We highlight the ambiguity of selecting a branch as discussed around Eq. (2.22) by explicitly keeping the product $\sqrt{y_{1} y_{2} y_{3} y_{4}}$ in the above expression.

The compact expression for the index, once the solutions to the BAE are known, is

$$
\begin{aligned}
& Z\left(y_{a}, \mathfrak{n}_{a}\right)=\left(\prod_{a=1}^{4} y_{a}^{-\frac{1}{2} N^{2} \mathfrak{n}_{a}}\right) y_{\phi_{1}}^{-\frac{1}{2} N^{2} \mathfrak{n}_{\phi_{1}}} y_{\phi_{2}}^{-\frac{1}{2} N^{2} \mathfrak{n}_{\phi_{2}}} \\
& \times \sum_{I \in B A E}\left[\frac{1}{\operatorname{det} \mathbb{B}} \frac{\prod_{i=1}^{N} x_{i}^{\frac{1}{2} N\left(6-\sum_{a=1}^{4} \mathfrak{n}_{a}-2 \mathfrak{n}_{\phi_{1}}\right)} \tilde{x}_{i}^{\frac{1}{2} N\left(6-\sum_{a=1}^{4} \mathfrak{n}_{a}-2 \mathfrak{n}_{\phi_{2}}\right)}}{\prod_{i, j=1}^{N} \prod_{a=1,2}\left(\tilde{x}_{j}-y_{a} x_{i}\right)^{1-\mathfrak{n}_{a}} \prod_{a=3,4}\left(x_{i}-y_{a} \tilde{x}_{j}\right)^{1-\mathfrak{n}_{a}}}\right. \\
& \left.\times \frac{\prod_{i \neq j}\left(1-\frac{x_{i}}{x_{j}}\right)\left(1-\frac{\tilde{x}_{i}}{\tilde{x}_{j}}\right)}{\prod_{i, j=1}^{N}\left(x_{i}-x_{j} y_{\phi_{1}}\right)^{1-\mathfrak{n}_{\phi_{1}}}\left(\tilde{x}_{j}-\tilde{x}_{i} y_{\phi_{2}}\right)^{1-\mathfrak{n}_{\phi_{2}}}}\right] .
\end{aligned}
$$

The transformation matrix $\mathbb{B}$ is given by

$$
\left.\mathbb{B}\right|_{\mathrm{BAEs}}=\left(\begin{array}{cc}
\mathbb{B}_{j l} & G_{j l} \\
-G_{l j} & \tilde{\mathbb{B}}_{j l}
\end{array}\right),
$$

where

$$
\begin{aligned}
\mathbb{B}_{j l}= & \delta_{j l}\left[k-\sum_{m=1}^{N} G_{j m}+x_{j} \sum_{m=1}^{N}\left(\frac{1-\delta_{j m} y_{\phi_{1}}}{x_{j}-x_{m} y_{\phi_{1}}}-\frac{\delta_{j m}-y_{\phi_{1}}}{x_{m}-x_{j} y_{\phi_{1}}}\right)\right] \\
& -\delta_{j l}\left[\frac{y_{\phi_{1}}+1}{y_{\phi_{1}}-1}\right]+x_{l}\left[\frac{1}{x_{l}-x_{j} y_{\phi_{1}}^{-1}}-\frac{1}{x_{l}-x_{j} y_{\phi_{1}}}\right], \\
\tilde{\mathbb{B}}_{j l}= & \delta_{j l}\left[k+\sum_{m=1}^{N} G_{m j}+\tilde{x}_{j} \sum_{m=1}^{N}\left(\frac{\delta_{j m}-y_{\phi_{2}}}{\tilde{x}_{m}-\tilde{x}_{j} y_{\phi_{2}}}-\frac{1-\delta_{j m} y_{\phi_{2}}}{\tilde{x}_{j}-\tilde{x}_{m} y_{\phi_{2}}}\right)\right] \\
& -\delta_{j l}\left[\frac{1+y_{\phi_{2}}}{1-y_{\phi_{2}}}\right]+\tilde{x}_{l}\left[\frac{1}{\tilde{x}_{l}-\tilde{x}_{j} y_{\phi_{2}}}-\frac{1}{\tilde{x}_{l}-\tilde{x}_{j} y_{\phi_{2}}^{-1}}\right] .
\end{aligned}
$$




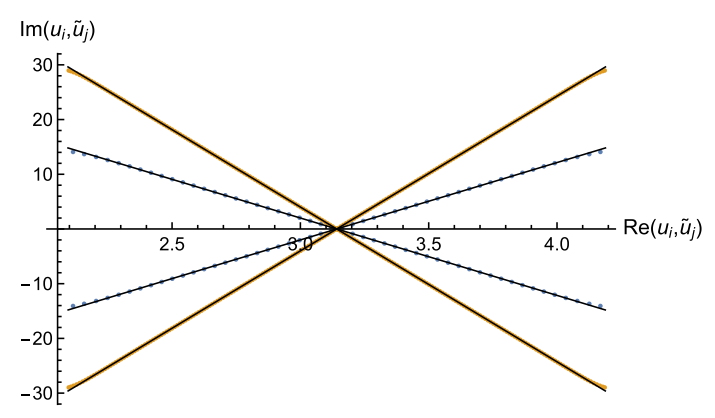

(a) Eigenvalue distribution

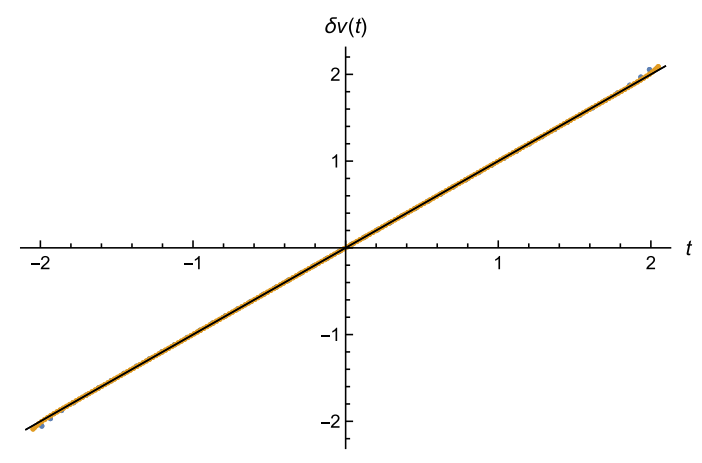

(c) Real part difference $\delta v(t)$

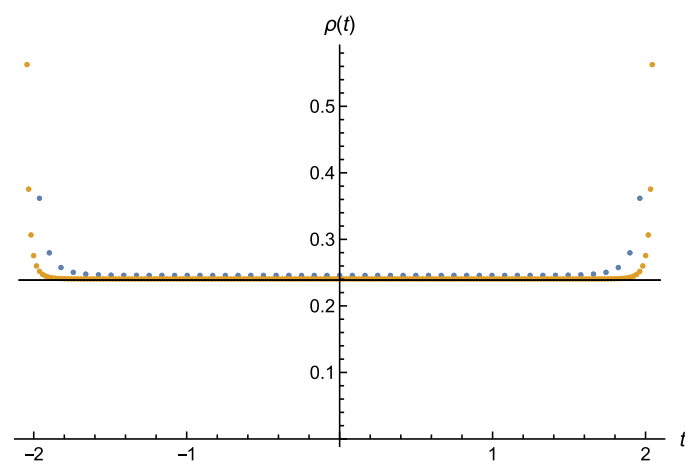

(b) Eigenvalue density $\rho(t)$

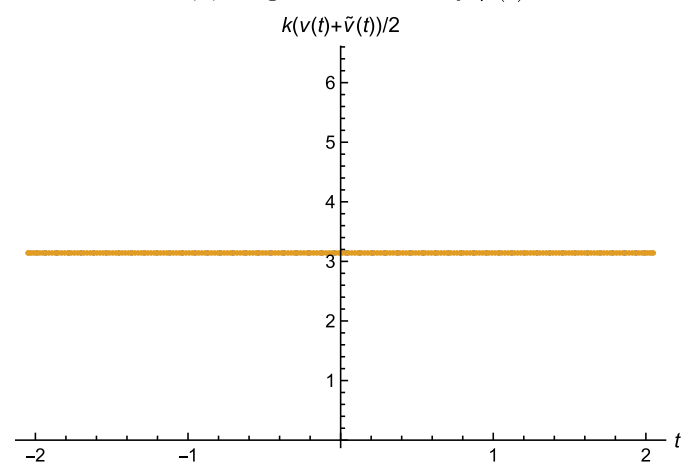

(d) Real part axis $k(v(t)+\tilde{v}(t)) / 2$
$N=50, k=1$,

$\Delta_{a}=\left\{\frac{2 \pi}{3}, \frac{2 \pi}{3}, \frac{2 \pi}{3}, \frac{2 \pi}{3}\right\}$,

$\left\{\Delta_{\phi_{1}}, \Delta_{\phi_{2}}\right\}=\left\{\frac{2 \pi}{3}, \frac{2 \pi}{3}\right\}$.

$N=200, k=1$,

$\Delta_{a}=\left\{\frac{2 \pi}{3}, \frac{2 \pi}{3}, \frac{2 \pi}{3}, \frac{2 \pi}{3}\right\}$,

$\left\{\Delta_{\phi_{1}}, \Delta_{\phi_{2}}\right\}=\left\{\frac{2 \pi}{3}, \frac{2 \pi}{3}\right\}$.

FIG. 7. Eigenvalues for the special case $\Delta_{a}=\left\{\frac{2 \pi}{3}, \frac{2 \pi}{3}, \frac{2 \pi}{3}, \frac{2 \pi}{3}\right\}$ for $N=50$ (blue) and 200 (orange) with the same other parameters.

The Bethe potential is

$$
\begin{aligned}
\mathcal{V}= & \sum_{i=1}^{N}\left[\frac{k}{2}\left(\tilde{u}_{i}^{2}-u_{i}^{2}\right)-2 \pi\left(\tilde{n}_{i} \tilde{u}_{i}-n_{i} u_{i}\right)\right] \\
& +\sum_{i, j=1}^{N}\left[\sum_{a=3,4} \operatorname{Li}_{2}\left(e^{i\left(\tilde{u}_{j}-u_{i}+\Delta_{a}\right)}\right)-\sum_{a=1,2} \operatorname{Li}_{2}\left(e^{i\left(\tilde{u}_{j}-u_{i}-\Delta_{a}\right)}\right)-\frac{1}{2}\left(\sum_{a=1}^{4} \Delta_{a}-2 \pi\right)\left(\tilde{u}_{j}-u_{i}\right)\right] \\
& +\sum_{i, j=1}^{N} \frac{1}{2}\left[\operatorname{Li}_{2}\left(e^{i\left(u_{j}-u_{i}+\Delta_{\phi_{1}}\right)}\right)-\operatorname{Li}_{2}\left(e^{i\left(u_{j}-u_{i}-\Delta_{\phi_{1}}\right)}\right)\right]+\sum_{i, j=1}^{N} \frac{1}{2}\left[\operatorname{Li}_{2}\left(e^{i\left(\tilde{u}_{j}-\tilde{u}_{i}+\Delta_{\phi_{2}}\right)}\right)-\operatorname{Li}_{2}\left(e^{i\left(\tilde{u}_{j}-\tilde{u}_{i}-\Delta_{\phi_{2}}\right)}\right)\right],
\end{aligned}
$$

where

$$
\begin{aligned}
\sum_{i=1}^{N}\left[-2 \pi\left(\tilde{n}_{i} \tilde{u}_{i}-n_{i} u_{i}\right)\right] & =\left(6 \pi-\sum_{a=1}^{4} \Delta_{a}-2 \Delta_{\phi_{2}}\right) \sum_{i>j}^{N} \tilde{u}_{j}-\left(6 \pi-\sum_{a=1}^{4} \Delta_{a}-2 \Delta_{\phi_{1}}\right) \sum_{i>j}^{N} u_{i} \\
& =2 \pi \sum_{i>j}^{N}\left(\tilde{u}_{j}-u_{i}\right) .
\end{aligned}
$$

We focus on the case $k=1$ and set the initial real part axis to be $\pi$. The numerical solutions for different values of $N$ and $\Delta_{a}=\left\{\Delta_{1}, \Delta_{2}, \Delta_{3}, \Delta_{4}\right\}$ are shown in Figs. 7 and 8. The main new ingredients, as compared to the ABJM case, are the fugacities $\left\{\Delta_{\phi_{1}}, \Delta_{\phi_{2}}\right\}=$ $\left\{\frac{2 \pi}{3}, \frac{2 \pi}{3}\right\}$.
Figure 7 displays the eigenvalues and their imaginary and real part densities as a function of $N$. The choice of $N=50$ and $N=200$ is meant to indicate clearly that the imaginary part of the eigenvalues scales as $N^{1 / 2}$ in the large $N$ limit; this can be easily noted by glancing at the axes in panel $(a)$ of Figure 7. 


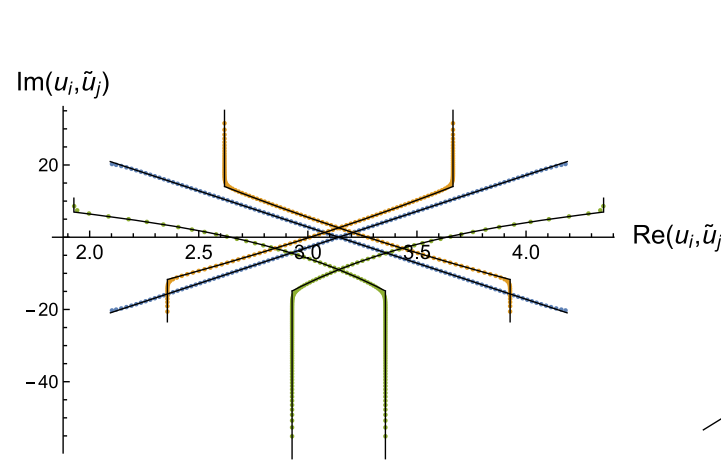

(a) Eigenvalue distribution

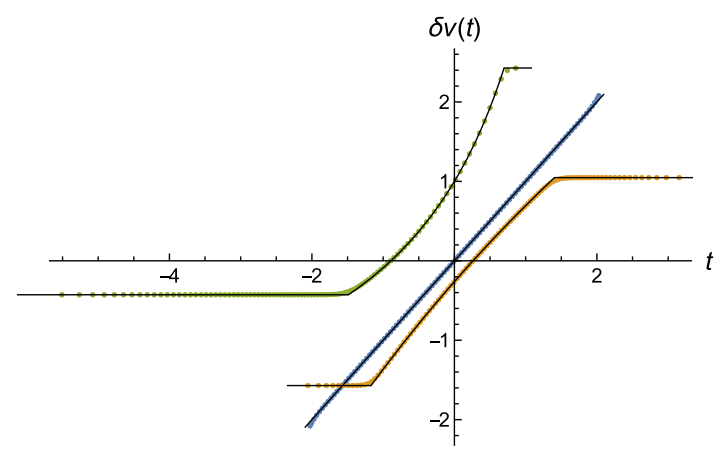

(c) Real part difference $\delta v(t)$

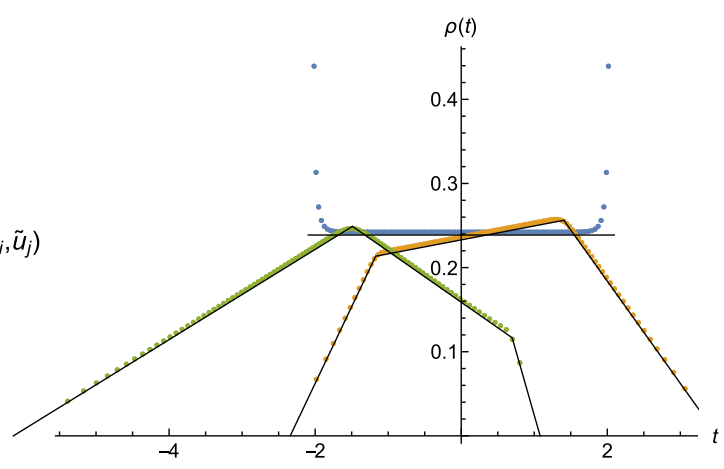

(b) Eigenvalue density $\rho(t)$

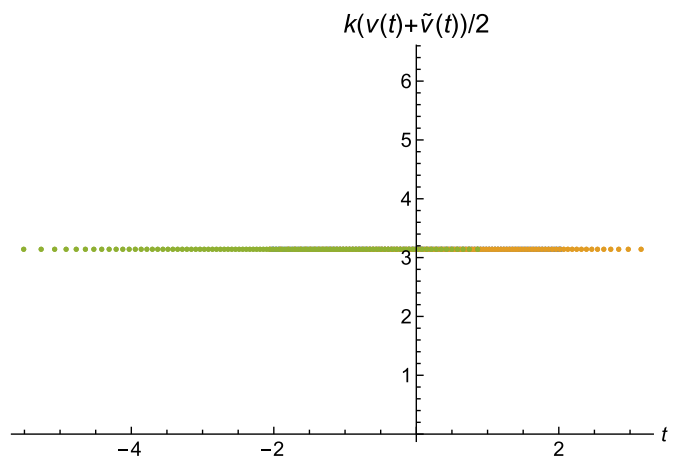

(d) Real part axis $k(v(t)+\tilde{v}(t)) / 2$

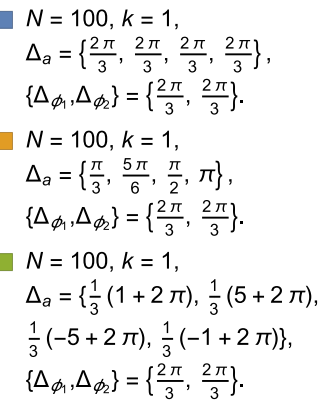

FIG. 8. Eigenvalues for the special case $\Delta_{a}=\left\{\frac{2 \pi}{3}, \frac{2 \pi}{3}, \frac{2 \pi}{3}, \frac{2 \pi}{3}\right\}$ (blue), and the general case $\Delta_{a}=\left\{\frac{\pi}{3}, \frac{5 \pi}{6}, \frac{\pi}{2}, \pi\right\}$ (orange) and $\Delta_{a}=$ $\left\{\frac{1}{3}+\frac{2 \pi}{3}, \frac{5}{3}+\frac{2 \pi}{3},-\frac{5}{3}+\frac{2 \pi}{3},-\frac{1}{3}+\frac{2 \pi}{3}\right\}$ (green) with the same other parameters.

Figure 8 explores the dependence of the eigenvalues on the choice of fugacities $\left\{\Delta_{a}\right\}$. As expected from the ABJM construction, and more general configuration of tails in the distribution of the eigenvalues emerges. The agreement with the leading order large $N$ distribution is maintained with deviations registered mostly along the piece-wise discontinuous slopes.

\section{The subleading term of the index at large $N$}

The index should take the form

$$
\begin{aligned}
\operatorname{Re} \log Z= & f_{1}(k, \Delta, \mathfrak{n}) N^{3 / 2}+f_{2}(k, \Delta, \mathfrak{n}) N^{1 / 2} \\
& +f_{3}(k, \Delta, \mathfrak{n}) \log N+f_{4}(k, \Delta, \mathfrak{n})+\mathcal{O}\left(N^{-1 / 2}\right),
\end{aligned}
$$

where the functions $f_{1}, f_{2}, f_{3}$ and $f_{4}$ are linear in the magnetic fluxes $\mathfrak{n}$.

The index Eq. (4.6) and $\operatorname{Re} \log Z$ can be computed using the numerical solutions. Under the similar decomposition

$$
\operatorname{Re} \log Z=A+B_{1} \mathfrak{n}_{3}+B_{2} \mathfrak{n}_{4},
$$

where we have used the marginality condition on the superpotential $\mathfrak{n}_{1}+\mathfrak{n}_{4}=4 / 3, \mathfrak{n}_{2}+\mathfrak{n}_{3}=4 / 3$ and $\mathfrak{n}_{\phi_{1}}=\mathfrak{n}_{\phi_{2}}=2 / 3$. Then we perform a linear least-square fit for $A$ and $B_{a}$ to the function

$$
\begin{aligned}
f(N)= & f_{1} N^{3 / 2}+f_{2} N^{1 / 2}+f_{3} \log N+f_{4} \\
& +\sum_{p=1}^{p_{c}} f_{p+4} N^{(1-2 p) / 2} .
\end{aligned}
$$

The results of the numerical fit for $\operatorname{Re} \log Z$ with $N$ are presented in Table III. The analytical leading term computed by the index theorem in [26] and the numerical leading term $f_{1} N^{3 / 2}$ match to number of significant digits present in the table. The numerical results indicate that the coefficient $f_{3}$ of the $\log N$ term is precisely $-1 / 2$.

\section{B. Model II}

Model II was originally proposed in [43] with the following quiver diagram:

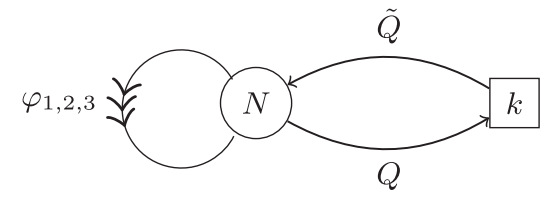

The superpotential is taken to be 
TABLE III. $\quad\left(V^{5,2}\right.$ Model I) Numerical fit for Relog $Z=f_{1} N^{3 / 2}+f_{2} N^{1 / 2}+f_{3} \log N+f_{4}+\sum_{p=1}^{p_{c}=5} f_{p+4} N^{(1-2 p) / 2}$.

\begin{tabular}{|c|c|c|c|c|}
\hline \multicolumn{5}{|c|}{ (a) $k=1 ;\left\{\Delta_{1}, \Delta_{2}, \Delta_{3}, \Delta_{4}\right\}=\left\{\frac{2 \pi}{3}, \frac{2 \pi}{3}, \frac{2 \pi}{3}, \frac{2 \pi}{3}\right\} ;\left\{\Delta_{\phi_{1}}, \Delta_{\phi_{2}}\right\}=\left\{\frac{2 \pi}{3}, \frac{2 \pi}{3}\right\}$} \\
\hline$N(s)$ & $f_{1}$ & $f_{2}$ & $f_{3}$ & $f_{4}$ \\
\hline $100 \sim 300(10)$ & -1.86168 & +3.02526 & -0.50066 & -2.75740 \\
\hline \multicolumn{5}{|c|}{ (b) $k=1 ;\left\{\Delta_{1}, \Delta_{2}, \Delta_{3}, \Delta_{4}\right\}=\left\{\frac{\pi}{3}, \frac{5 \pi}{6}, \frac{\pi}{2}, \pi\right\} ;\left\{\Delta_{\phi_{1}}, \Delta_{\phi_{2}}\right\}=\left\{\frac{2 \pi}{3}, \frac{2 \pi}{3}\right\}$} \\
\hline$N(s)$ & $f_{1}$ & $f_{2}$ & $f_{3}$ & $f_{4}$ \\
\hline $100 \sim 300(10)$ & $\begin{array}{l}-2.18550 \\
-0.31221 \mathfrak{n}_{3} \\
+0.78053 \mathfrak{n}_{4}\end{array}$ & $\begin{array}{l}+3.56708 \\
+0.00781 \mathfrak{n}_{3} \\
-0.17562 \mathfrak{n}_{4}\end{array}$ & -0.50082 & $\begin{array}{l}-3.39532 \\
-0.02821 \mathfrak{n}_{3} \\
+0.19180 \mathfrak{n}_{4}\end{array}$ \\
\hline \multicolumn{5}{|c|}{ (c) $k=1 ;\left\{\Delta_{1}, \Delta_{2}, \Delta_{3}, \Delta_{4}\right\}=\left\{\frac{2 \pi}{3}+\frac{1}{3}, \frac{2 \pi}{3}+\frac{5}{3}, \frac{2 \pi}{3}-\frac{5}{3}, \frac{2 \pi}{3}-\frac{1}{3}\right\} ;\left\{\Delta_{\phi_{1}}, \Delta_{\phi_{2}}\right\}=\left\{\frac{2 \pi}{3}, \frac{2 \pi}{3}\right\}$} \\
\hline$N(s)$ & $f_{1}$ & $f_{2}$ & $f_{3}$ & $f_{4}$ \\
\hline $100 \sim 200(5)$ & $\begin{array}{l}-0.79002 \\
-1.81136 \mathfrak{n}_{3} \\
-0.13631 \mathfrak{n}_{4}\end{array}$ & $\begin{array}{l}+4.27652 \\
+1.38166 \mathfrak{n}_{3} \\
-0.06990 \mathfrak{n}_{4}\end{array}$ & $\begin{array}{l}-0.50167 \\
-0.00005 \mathfrak{n}_{3} \\
-0.00001 \mathfrak{n}_{4}\end{array}$ & $\begin{array}{l}-4.51009 \\
-1.91182 \mathfrak{n}_{3} \\
+0.01935 \mathfrak{n}_{4}\end{array}$ \\
\hline
\end{tabular}

$W=\operatorname{Tr}\left\{\varphi_{3}\left[\varphi_{1}, \varphi_{2}\right]+\sum_{j=1}^{k} q_{j}\left(\varphi_{1}^{2}+\varphi_{2}^{2}+\varphi_{3}^{2}\right) \tilde{q}^{j}\right\}$

The $S O(5)$ symmetry of $V^{5,2}$ can be made manifest by using the variables

$X_{1}=\frac{1}{\sqrt{2}}\left(\varphi_{1}+i \varphi_{2}\right), \quad X_{2}=\frac{1}{\sqrt{2}}\left(\varphi_{1}-i \varphi_{2}\right), \quad X_{3}=i \varphi_{3}$.

In terms of these new variables, the superpotential can be rewritten as

$W=\operatorname{Tr}\left\{X_{3}\left[X_{1}, X_{2}\right]+\sum_{j=1}^{k} q_{j}\left(X_{1} X_{2}+X_{2} X_{1}-X_{3}^{2}\right) \tilde{q}^{j}\right\}$.

\section{Numerical solutions to the system of BAEs}

The above matter content implies that the topologically twisted index takes the general form

$$
\begin{aligned}
Z= & \frac{1}{N !} \sum_{\mathfrak{m} \in \mathbb{Z}^{N}} \int_{\mathcal{C}} \prod_{i=1}^{N} \frac{d x_{i}}{2 \pi i x_{i}} x_{i}^{\mathrm{t}} \xi^{\mathfrak{m}_{i}} \times \prod_{i \neq j}^{N}\left(1-\frac{x_{i}}{x_{j}}\right) \\
& \times \prod_{i, j=1}^{N} \prod_{a=1}^{3}\left(\frac{\sqrt{\frac{x_{i}}{x_{j}} y_{X_{a}}}}{1-\frac{x_{i}}{x_{j}} y_{X_{a}}}\right)^{\mathfrak{m}_{i}-\frac{1}{2} \mathfrak{n}_{X_{a}}+\frac{1}{2}}\left(\frac{\sqrt{\frac{x_{j}}{x_{i}} y_{X_{a}}}}{1-\frac{x_{j}}{x_{i}} y_{X_{a}}}\right)^{-\mathfrak{m}_{i}-\frac{1}{2} \mathfrak{n}_{X_{a}}+\frac{1}{2}} \\
& \times \prod_{i=1}^{N} \prod_{j=1}^{k}\left(\frac{\sqrt{x_{i} y_{q_{j}}}}{1-x_{i} y_{q_{j}}}\right)^{\mathfrak{m}_{i}-\mathfrak{n}_{q_{j}}+1}\left(\frac{\sqrt{\frac{1}{x_{i}} \tilde{y}_{q_{j}}}}{1-\frac{1}{x_{i}} \tilde{y}_{q_{j}}}\right)^{-\mathfrak{m}_{i}-\tilde{\mathfrak{n}}_{q_{j}}+1}
\end{aligned}
$$

Performing the summation over magnetic fluxes by introducing a large cutoff $M$ we get

$$
\begin{aligned}
Z= & \frac{1}{N !} \sum_{\mathfrak{m} \in \mathbb{Z}^{N}} \int_{\mathcal{C}} \prod_{i=1}^{N} \frac{d x_{i}}{2 \pi i x_{i}} x_{i}^{\mathrm{t}} \times \prod_{i \neq j}^{N}\left(1-\frac{x_{i}}{x_{j}}\right) \prod_{i, j=1}^{N} \prod_{a=1}^{3}\left(\frac{\sqrt{y_{X_{a}}}}{1-\frac{x_{j}}{x_{i}} y_{X_{a}}}\right)^{1-\mathfrak{n}_{X_{a}}} \\
& \times \prod_{i=1}^{N} \prod_{j=1}^{k}\left(\frac{\sqrt{x_{i} y_{q_{j}}}}{1-x_{i} y_{q_{j}}}\right)^{1-\mathfrak{n}_{q_{j}}}\left(\frac{\sqrt{\frac{1}{x_{i}} \tilde{y}_{q_{j}}}}{1-\frac{1}{x_{i}} \tilde{y}_{q_{j}}}\right)^{1-\mathfrak{n}_{q_{j}}} \prod_{i=1}^{N} \frac{\left(e^{i B_{i}}\right)^{M}}{e^{i B_{i}}-1}
\end{aligned}
$$

where the Bethe Ansatz equations are

$$
1=e^{i B_{i}}=\xi \prod_{a=1}^{3} \prod_{j=1}^{N}\left(\frac{x_{i}-x_{j} y_{X_{a}}}{x_{j}-x_{i} y_{X_{a}}}\right) \prod_{j=1}^{k}\left(\frac{\sqrt{x_{i} y_{q_{j}}}}{1-x_{i} y_{q_{j}}}\right)\left(\frac{\sqrt{\frac{1}{x_{i}} \tilde{y}_{q_{j}}}}{1-\frac{1}{x_{i}} \tilde{y}_{q_{j}}}\right)^{-1} .
$$


The compact expression for the index once the BA solutions are known is

$$
\begin{aligned}
Z\left(y_{a}, \mathfrak{n}_{a}\right)= & e^{i \frac{\pi}{3} N k} \prod_{a=1}^{3} y_{X_{a}}^{-\frac{1}{2} N^{2} \mathfrak{n}_{X_{a}}} \prod_{j=1}^{k} y_{q_{j}}^{-\frac{1}{2} N \mathfrak{n}_{q_{j}}} \tilde{y}_{q_{j}}^{-\frac{1}{2} N \tilde{\mathfrak{n}}_{q_{j}}} \\
& \times \sum_{I \in B A E}\left[\frac{1}{\operatorname{det} \mathbb{B}} \frac{\prod_{i=1}^{N} x_{i}^{N+\mathfrak{t}} \prod_{i \neq j}\left(1-\frac{x_{i}}{x_{j}}\right)}{\prod_{i, j=1}^{N} \prod_{a=1}^{3}\left(x_{i}-x_{j} y_{X_{a}}\right)^{1-\mathfrak{n}_{X_{a}}}} \prod_{i=1}^{N} \frac{x_{i}^{\frac{2}{3} k}}{\prod_{j=1}^{k}\left(1-x_{i} y_{q_{j}}\right)^{\left(1-\mathfrak{n}_{q_{j}}\right)}\left(x_{i}-\tilde{y}_{q_{j}}\right)^{\left(1-\tilde{\mathfrak{n}}_{q_{j}}\right)}}\right] .
\end{aligned}
$$

The matrix $\mathbb{B}$ is

$$
\left.\mathbb{B}\right|_{\mathrm{BAEs}}=\left(\mathbb{B}_{j l}\right),
$$

where

$$
\begin{aligned}
\mathbb{B}_{j l}= & \delta_{j l}\left[x_{j} \sum_{a=1}^{3} \sum_{m=1}^{N}\left(\frac{1-\delta_{j m} y_{X_{a}}}{x_{j}-x_{m} y_{X_{a}}}-\frac{\delta_{j m}-y_{X_{a}}}{x_{m}-x_{j} y_{X_{a}}}\right)+x_{j} \sum_{i=1}^{k}\left(\frac{1}{x_{j}-\tilde{y}_{q_{i}}}-\frac{1}{x_{j}-y_{q_{i}}^{-1}}\right)\right] \\
& -\delta_{j l}\left[\sum_{a=1}^{3} \frac{y_{X_{a}}+1}{y_{X_{a}}-1}\right]+x_{l} \sum_{a=1}^{3}\left[\frac{1}{x_{l}-x_{j} y_{X_{a}}^{-1}}-\frac{1}{x_{l}-x_{j} y_{X_{a}}}\right]
\end{aligned}
$$

The Bethe potential is

$$
\begin{aligned}
\mathcal{V}= & \sum_{i=1}^{N}\left[-\Delta_{m} u_{i}+2 \pi n_{i} u_{i}\right]+\frac{1}{2} \sum_{a=1}^{3} \sum_{i, j=1}^{N}\left[\operatorname{Li}_{2}\left(e^{i\left(u_{j}-u_{i}+\Delta_{X_{a}}\right)}\right)-\operatorname{Li}_{2}\left(e^{i\left(u_{j}-u_{i}-\Delta_{X_{a}}\right)}\right)\right] \\
& +\sum_{i=1}^{N} \sum_{j=1}^{k}\left[\operatorname{Li}_{2}\left(e^{i\left(-u_{i}+\tilde{\Delta}_{q_{j}}\right)}\right)-\operatorname{Li}_{2}\left(e^{i\left(-u_{i}-\Delta_{q_{j}}\right.}\right)\right]+\frac{1}{2} \sum_{i=1}^{N} \sum_{j=1}^{k}\left[\left(\tilde{\Delta}_{q_{j}}+\Delta_{q_{j}}-2 \pi\right) u_{i}\right],
\end{aligned}
$$

where

$$
\sum_{i=1}^{N} 2 \pi n_{i} u_{i}=\left(3 \pi-\sum_{a=1}^{3} \Delta_{X_{a}}\right) \sum_{i>j}^{N}\left(u_{j}-u_{i}\right)=\pi \sum_{i>j}^{N}\left(u_{j}-u_{i}\right) .
$$

Notice that for even $N$ we are left with a common factor $\pi \sum_{i=1}^{N} u_{i}$ which can be reabsorbed in the definition of the topological fugacity $\xi$ [25].

For simplicity we set $\Delta_{q_{j}}=\Delta_{q}$ and $\tilde{\Delta}_{q_{j}}=\tilde{\Delta}_{q}$ for all $j$. Since there is only one gauge group we directly set the initial real part to be

$$
v(t)=\frac{\tilde{\Delta}_{q}-\Delta_{q}}{2} .
$$

The quiver described in quiver diagram (4.15) is quite different from the ABJM quiver, in particular, it has only one node. We have only one set of eigenvalues to consider. The set of fugacities involved $y_{X_{a}}, y_{q_{j}}$ and $\tilde{y}_{q_{j}}$ are

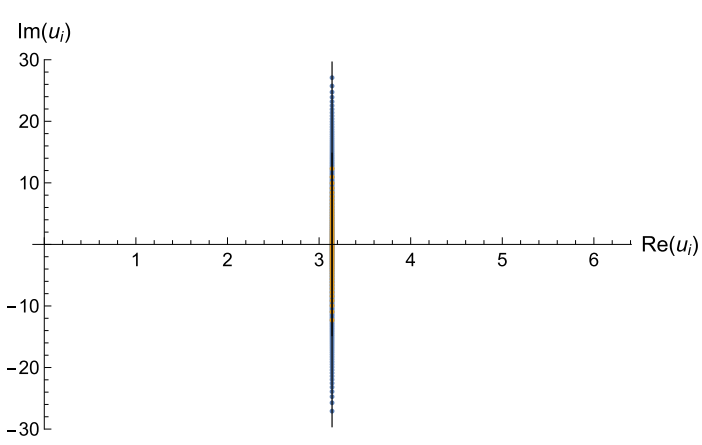

(a) Eigenvalue distribution

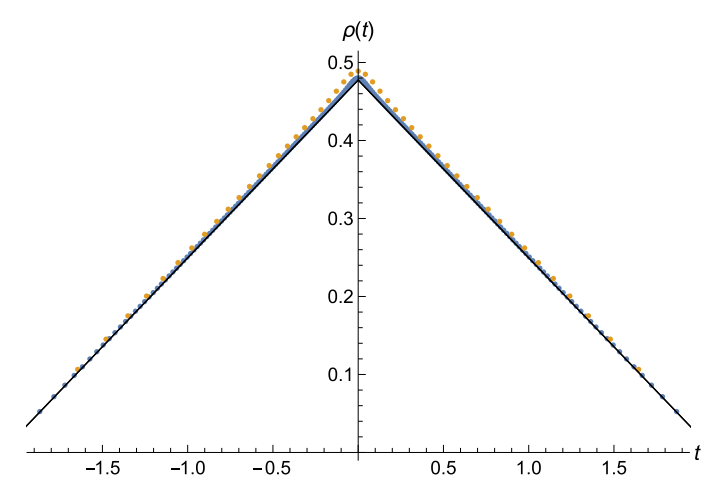

(b) Eigenvalue density $\rho(t)$

$$
\begin{aligned}
& N=200, \Delta_{m}=0, \\
& \Delta_{X}=\left\{\frac{2 \pi}{3}, \frac{2 \pi}{3}, \frac{2 \pi}{3}\right\}, \\
& k=1, \\
& \left\{\Delta_{q_{j}}, \tilde{\Delta}_{q j}\right\}=\left\{-\frac{2 \pi}{3}, \frac{4 \pi}{3}\right\} \\
& N=50, \Delta_{m}=0, \\
& \Delta_{X}=\left\{\frac{2 \pi}{3}, \frac{2 \pi}{3}, \frac{2 \pi}{3}\right\}, \\
& k=1 \\
& \left\{\Delta_{q_{j}}, \tilde{\Delta}_{q j}\right\}=\left\{-\frac{2 \pi}{3}, \frac{4 \pi}{3}\right\} .
\end{aligned}
$$

FIG. 9. Eigenvalues for the special case $\Delta_{X}=\left\{\frac{2 \pi}{3}, \frac{2 \pi}{3}, \frac{2 \pi}{3}\right\}$ for $N=50$ (orange) and 200 (blue) with the same other parameters. 


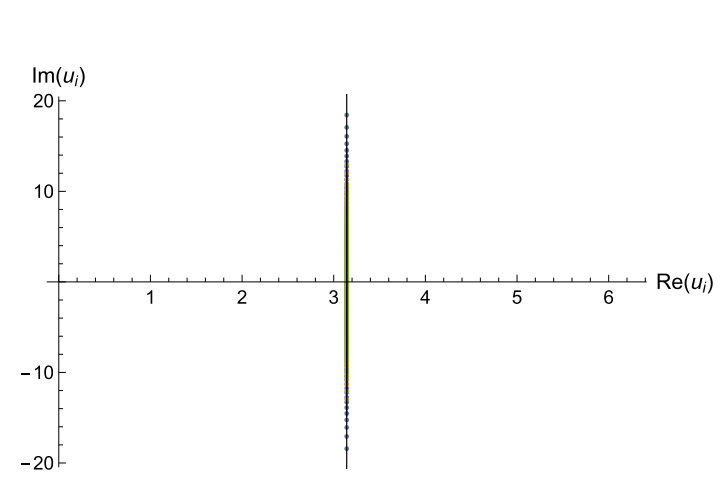

(a) Eigenvalue distribution

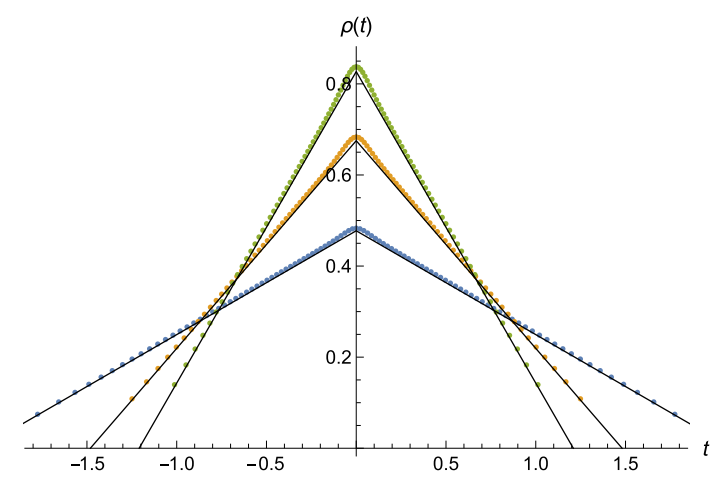

(b) Eigenvalue density $\rho(t)$
$N=100, \Delta_{m}=0$

$\Delta_{X}=\left\{\frac{2 \pi}{3}, \frac{2 \pi}{3}, \frac{2 \pi}{3}\right\}$,

$k=1$,

$\left\{\Delta_{q_{j}}, \tilde{\Delta}_{q_{j}}\right\}=\left\{-\frac{2 \pi}{3}, \frac{4 \pi}{3}\right\}$.

$N=100, \Delta_{m}=0$,

$\Delta_{X}=\left\{\frac{2 \pi}{3}, \frac{2 \pi}{3}, \frac{2 \pi}{3}\right\}$,

$k=2$,

$\left\{\Delta_{q_{j}}, \tilde{\Delta}_{q_{j}}\right\}=\left\{-\frac{2 \pi}{3}, \frac{4 \pi}{3}\right\}$.

$N=100, \Delta_{m}=0$,

$\Delta_{X}=\left\{\frac{2 \pi}{3}, \frac{2 \pi}{3}, \frac{2 \pi}{3}\right\}$,

$k=3$,

$\left\{\Delta_{q_{j}}, \tilde{\Delta}_{q_{j}}\right\}=\left\{-\frac{2 \pi}{3}, \frac{4 \pi}{3}\right\}$.

FIG. 10. Eigenvalues for the special case $\Delta_{X}=\left\{\frac{2 \pi}{3}, \frac{2 \pi}{3}, \frac{2 \pi}{3}\right\}$ for $k=1$ (blue), 2 (orange) and 3 (green) with the same other parameters.

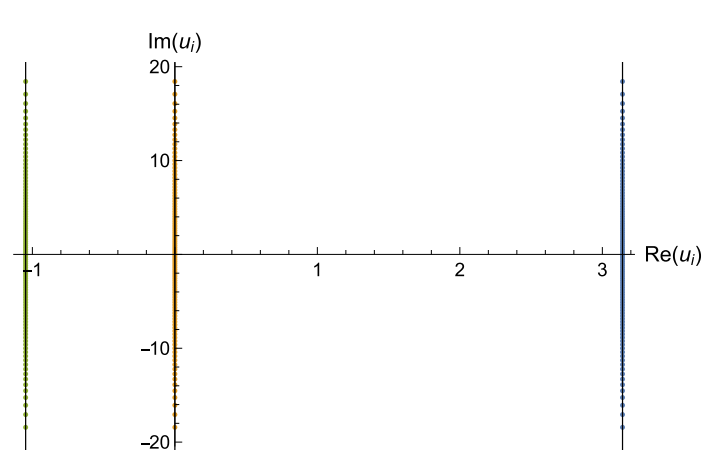

(a) Eigenvalue distribution

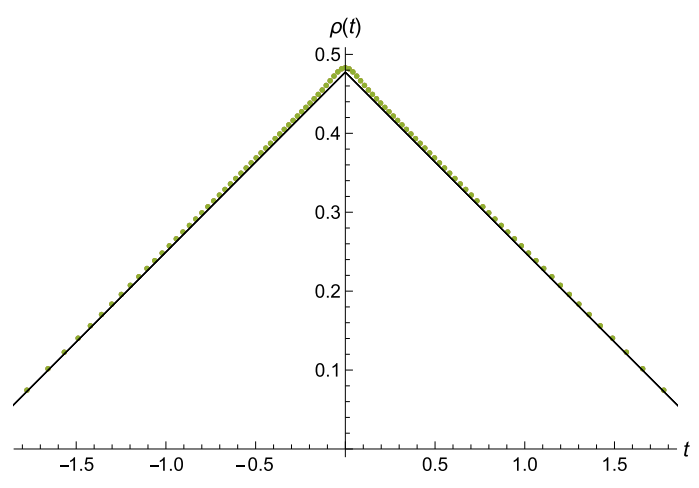

(b) Eigenvalue density $\rho(t)$
$N=100, \Delta_{m}=0$

$\Delta_{X}=\left\{\frac{2 \pi}{3}, \frac{2 \pi}{3}, \frac{2 \pi}{3}\right\}$,

$k=1$,

$\left\{\Delta_{q_{j}}, \tilde{\Delta}_{q_{j}}\right\}=\left\{-\frac{2 \pi}{3}, \frac{4 \pi}{3}\right\}$.

$N=100, \Delta_{m}=0$,

$\Delta_{X}=\left\{\frac{2 \pi}{3}, \frac{2 \pi}{3}, \frac{2 \pi}{3}\right\}$,

$k=1$,

$\left\{\Delta_{q_{j}}, \tilde{\Delta}_{q_{j}}\right\}=\left\{\frac{\pi}{3}, \frac{\pi}{3}\right\}$.

$N=100, \Delta_{m}=0$,

$\Delta_{X}=\left\{\frac{2 \pi}{3}, \frac{2 \pi}{3}, \frac{2 \pi}{3}\right\}$,

$k=1$,

$\left\{\Delta_{q_{j}}, \tilde{\Delta}_{q_{j}}\right\}=\left\{\frac{2 \pi}{3}, 0\right\}$.

FIG. 11. Eigenvalues for the special case $\Delta_{X}=\left\{\frac{2 \pi}{3}, \frac{2 \pi}{3}, \frac{2 \pi}{3}\right\}$ for $\left\{\Delta_{q}, \tilde{\Delta}_{q}\right\}=\left\{-\frac{2 \pi}{3}, \frac{4 \pi}{3}\right\}$ (blue), $\left\{\frac{\pi}{3}, \frac{\pi}{3}\right\}$ (orange) and $\left\{\frac{2 \pi}{3}, 0\right\}$ (green) with the same other parameters.

quite different as well, in the sense that there is no particular subset that one would naturally identify with the $y_{a}$ of the ABJM model.

The numerical solutions for different values of $N, k$, $\left\{\Delta_{q}, \tilde{\Delta}_{q}\right\}$ and $\Delta_{m}, \Delta_{X}=\left\{\Delta_{X_{1}}, \Delta_{X_{2}}, \Delta_{X_{3}}\right\}$ are shown in
Figs. 9-12. We find the value of the real parts of the exact eigenvalues is the same as our initial value.

Figure 9 shows that the real part of the eigenvalues is a constant. It is harder to spot the scaling from panel (a) but it is still $N^{1 / 2}$.

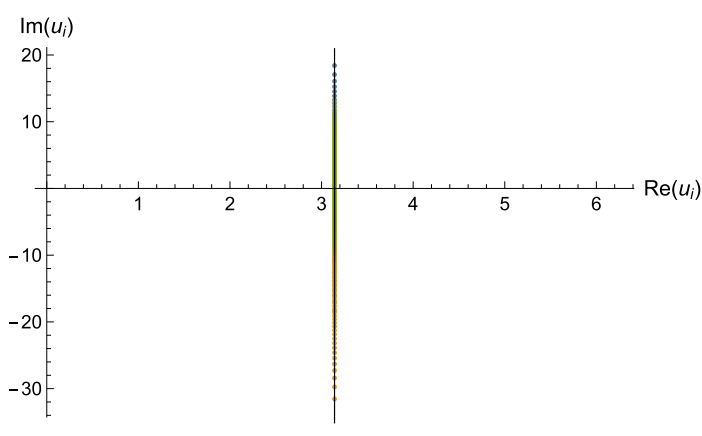

(a) Eigenvalue distribution

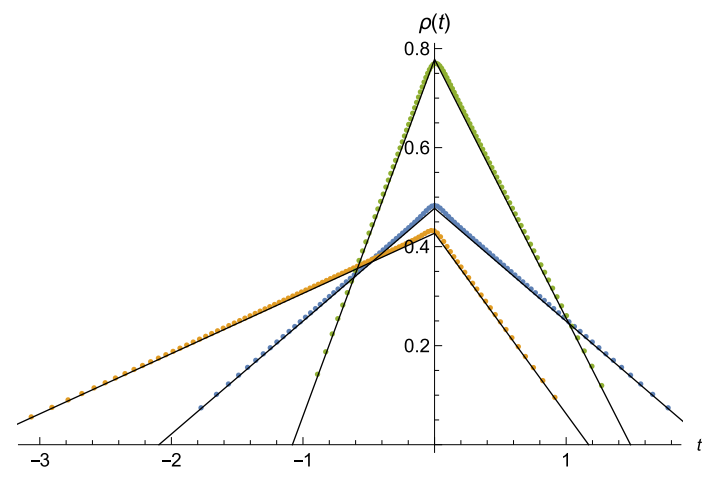

(b) Eigenvalue density $\rho(t)$
$N=100, \Delta_{m}=0$,
$\Delta_{X}=\left\{\frac{2 \pi}{3}, \frac{2 \pi}{3}, \frac{2 \pi}{3}\right\}$,
$k=1$,
$\left\{\Delta_{q_{j}}, \tilde{\Delta}_{q_{j}}\right\}=\left\{-\frac{2 \pi}{3}, \frac{4 \pi}{3}\right\}$.
$N=100, \Delta_{m}=-\frac{\pi}{3}$,
$\Delta_{X}=\left\{\frac{\pi}{2}, \frac{5 \pi}{6}, \frac{2 \pi}{3}\right\}$,
$k=1$,
$\left\{\Delta_{q_{j}}, \tilde{\Delta}_{q_{j}}\right\}=\left\{-\frac{2 \pi}{3}, \frac{4 \pi}{3}\right\}$.
$N=100, \Delta_{m}=\frac{1}{3}$,
$\Delta_{X}=\left\{\frac{1}{3}(-5+2 \pi)\right.$,
$\left.\frac{1}{3}(5+2 \pi), \frac{2 \pi}{3}\right\}$,
$k=1$,

$\left\{\Delta_{q_{j}}, \tilde{\Delta}_{q_{j}}\right\}=\left\{-\frac{2 \pi}{3}, \frac{4 \pi}{3}\right\}$.

FIG. 12. Eigenvalues for the special case $\Delta_{m}=0, \Delta_{X}=\left\{\frac{2 \pi}{3}, \frac{2 \pi}{3}, \frac{2 \pi}{3}\right\}$ (blue), and for the general cases $\Delta_{m}=-\frac{\pi}{3}, \Delta_{X}=\left\{\frac{\pi}{2}, \frac{5 \pi}{6}, \frac{2 \pi}{3}\right\}$ (orange) and $\Delta_{m}=\frac{1}{3}, \Delta_{X}=\left\{-\frac{5}{3}+\frac{2 \pi}{3}, \frac{5}{3}+\frac{2 \pi}{3}, \frac{2 \pi}{3}\right\}$ (green) with the same other parameters. 
In Fig. 10 we consider the effect of changing the number of flavors $k$. As expected from the leading order solution [26], changing $k$ mostly affects the slope in the eigenvalue distribution $\rho(t)$ which increases proportional to $k$.

Figure 11 demonstrates that the role of the chemical potentials $\left\{\Delta_{q}, \tilde{\Delta}_{q}\right\}$ display the real part of the eigenvalues $u_{i}$ and have, otherwise no effect on the imaginary eigenvalue density $\rho(t)$.

Finally, we display the dependence of the eigenvalues $u_{i}$ on the choice of the chemical potential $\Delta_{X}$ in Fig. 12 keeping the same real parts.

\section{The subleading term of the index at large $N$}

The index should take the form

$\operatorname{Re} \log Z=f_{1}(k, \Delta, \mathfrak{n}, \mathfrak{t}) N^{3 / 2}+f_{2}(k, \Delta, \mathfrak{n}, \mathfrak{t}) N^{1 / 2}$

$$
\begin{aligned}
& +f_{3}(k, \Delta, \mathfrak{n}, \mathfrak{t}) \log N \\
& +f_{4}(k, \Delta, \mathfrak{n}, \mathfrak{t})+\mathcal{O}\left(N^{-1 / 2}\right),
\end{aligned}
$$

where the functions $f_{1}, f_{2}, f_{3}$ and $f_{4}$ are linear in the magnetic fluxes $\mathfrak{n}$ and $\mathfrak{t}$.

\begin{tabular}{|c|c|c|c|c|c|c|c|}
\hline \multicolumn{8}{|c|}{ (a) $\Delta_{m}=0 ;\left\{\Delta_{X_{1}}, \Delta_{X_{2}}, \Delta_{X_{3}}\right\}=\left\{\frac{2 \pi}{3}, \frac{2 \pi}{3}, \frac{2 \pi}{3}\right\}$} \\
\hline$k$ & $\Delta_{q}$ & $\tilde{\Delta}_{q}$ & $N(s)$ & $f_{1}$ & $f_{2}$ & $f_{3}$ & $f_{4}$ \\
\hline & $-\frac{2 \pi}{3}$ & $\frac{4 \pi}{3}$ & $100 \sim 300(10)$ & -1.86168 & +3.02526 & -0.50066 & -2.75740 \\
\hline 1 & $\frac{\pi}{3}$ & $\frac{\pi}{3}$ & $100 \sim 300(10)$ & -1.86168 & +3.02526 & -0.50066 & -2.75740 \\
\hline & $\frac{2 \pi}{3}$ & 0 & $100 \sim 300(10)$ & -1.86168 & +3.02526 & -0.50066 & -2.75740 \\
\hline 2 & $-\frac{2 \pi}{3}$ & $\frac{4 \pi}{3}$ & $100 \sim 200(5)$ & -2.63282 & +3.37334 & -0.50090 & -3.26096 \\
\hline 3 & $-\frac{2 \pi}{3}$ & $\frac{3}{3}$ & $100 \sim 200(5)$ & -3.22453 & +4.43378 & -0.50115 & -4.75453 \\
\hline
\end{tabular}

TABLE IV. $\quad\left(V^{5,2}\right.$ Model II) Numerical fit for $\operatorname{Re} \log Z=f_{1} N^{3 / 2}+f_{2} N^{1 / 2}+f_{3} \log N+f_{4}+\sum_{g=1}^{g_{c}=5} N^{(1-2 g) / 2}$.

\begin{tabular}{|c|c|c|c|c|c|c|c|}
\hline$k$ & $\Delta_{q}$ & $\tilde{\Delta}_{q}$ & $N(s)$ & $f_{1}$ & $f_{2}$ & $f_{3}$ & $f_{4}$ \\
\hline \multirow[t]{3}{*}{1} & $-\frac{2 \pi}{3}$ & $\frac{4 \pi}{3}$ & $100 \sim 300(10)$ & $\begin{array}{l}-1.66514 \\
-0.31221 \mathfrak{n}_{X_{1}} \\
+0.78053 \mathfrak{t}\end{array}$ & $\begin{array}{l}+3.44999 \\
+0.00781 \mathfrak{n}_{X_{1}} \\
-0.17562 \mathfrak{t}\end{array}$ & -0.50082 & $\begin{array}{l}-3.26746 \\
-0.02821 \mathfrak{n}_{X_{1}} \\
+0.19180 \mathrm{t}\end{array}$ \\
\hline & $\frac{\pi}{3}$ & $\frac{\pi}{3}$ & $100 \sim 300(10)$ & $\begin{array}{l}-1.66514 \\
-0.31221 \mathfrak{n}_{X_{1}} \\
+0.78053 \mathfrak{t}\end{array}$ & $\begin{array}{l}+3.44999 \\
+0.00781 \mathfrak{n}_{X_{1}} \\
-0.17562 \mathfrak{t}\end{array}$ & -0.50082 & $\begin{array}{l}-3.26746 \\
-0.02821 \mathfrak{n}_{X_{1}} \\
+0.19180 \mathrm{t}\end{array}$ \\
\hline & $\frac{2 \pi}{3}$ & 0 & $100 \sim 300(10)$ & $\begin{array}{l}-1.66514 \\
-0.31221 \mathfrak{n}_{X_{1}} \\
+0.78053 \mathrm{t}\end{array}$ & $\begin{array}{l}+3.44999 \\
+0.00781 \mathfrak{n}_{X_{1}} \\
-0.17562 \mathrm{t}\end{array}$ & -0.50082 & $\begin{array}{l}-3.26746 \\
-0.02821 \mathfrak{n}_{X_{1}} \\
+0.19180 \mathrm{t}\end{array}$ \\
\hline 2 & $-\frac{2 \pi}{3}$ & $\frac{4 \pi}{3}$ & $100 \sim 200(5)$ & $\begin{array}{l}-2.30372 \\
-0.49365 \mathfrak{n}_{X_{1}} \\
+0.24683 \mathfrak{t}\end{array}$ & $\begin{array}{l}+3.19233 \\
+0.41961 \mathfrak{n}_{X_{1}} \\
+0.08639 \mathfrak{t}\end{array}$ & -0.50098 & $\begin{array}{l}-3.22095 \\
-0.34133 \mathfrak{n}_{X_{1}} \\
-0.06902 \mathfrak{t}\end{array}$ \\
\hline 3 & $-\frac{2 \pi}{3}$ & $\frac{4 \pi}{3}$ & $100 \sim 200(5)$ & $\begin{array}{l}-2.81460 \\
-0.61569 \mathfrak{n}_{X_{1}} \\
+0.13193 \mathfrak{t}\end{array}$ & $\begin{array}{l}+3.99013 \\
+0.89056 \mathfrak{n}_{X_{1}} \\
+0.09188 \mathfrak{t}\end{array}$ & $\begin{array}{l}-0.50123 \\
-0.00006 \mathfrak{n}_{X_{1}} \\
+0.00003 \mathfrak{t}\end{array}$ & $\begin{array}{l}-4.52640 \\
-0.83862 \mathfrak{n}_{X_{1}} \\
-0.08196 \mathfrak{t}\end{array}$ \\
\hline
\end{tabular}

(b) $\Delta_{m}=-\frac{\pi}{3} ;\left\{\Delta_{X_{1}}, \Delta_{X_{2}}, \Delta_{X_{3}}\right\}=\left\{\frac{\pi}{2}, \frac{5 \pi}{6}, \frac{2 \pi}{3}\right\}$

(c) $\Delta_{m}=\frac{1}{3} ;\left\{\Delta_{X_{1}}, \Delta_{X_{2}}, \Delta_{X_{3}}\right\}=\left\{\frac{2 \pi}{3}-\frac{5}{3}, \frac{2 \pi}{3}+\frac{5}{3}, \frac{2 \pi}{3}\right\}$

\begin{tabular}{ccccllll}
\hline$k$ & $\Delta_{q}$ & $\tilde{\Delta}_{q}$ & $N(s)$ & \multicolumn{1}{c}{$f_{1}$} & \multicolumn{1}{c}{$f_{2}$} & \multicolumn{1}{c}{$f_{3}$} & \multicolumn{1}{c}{$f_{4}$} \\
\hline 1 & $-\frac{2 \pi}{3}$ & $\frac{4 \pi}{3}$ & $100 \sim 200(5)$ & -0.88090 & +4.22992 & -0.50168 & -4.49720 \\
& & & & $-1.81136 \mathfrak{n}_{X_{1}}$ & $+1.38166 \mathfrak{n}_{X_{1}}$ & $-0.00005 \mathfrak{n}_{X_{1}}$ & $-1.91182 \mathfrak{n}_{X_{1}}$ \\
& & & $-0.13631 \mathrm{t}$ & $-0.06990 \mathrm{t}$ & $-0.00001 \mathrm{t}$ & $+0.01935 \mathrm{t}$ \\
2 & $-\frac{2 \pi}{3}$ & $\frac{4 \pi}{3}$ & $100 \sim 200(5)$ & -1.24227 & +3.87599 & -0.50409 & -5.28616 \\
& & & & $-2.58650 \mathfrak{n}_{X_{1}}$ & $+5.44770 \mathfrak{n}_{X_{1}}$ & $+0.00620 \mathfrak{n}_{X_{1}}$ & $-8.41742 \mathfrak{n}_{X_{1}}$ \\
& & & $-0.04773 \mathrm{t}$ & $-0.07670 \mathrm{t}$ & $+0.00063 \mathrm{t}$ & $+0.05162 \mathrm{t}$ \\
3 & $-\frac{2 \pi}{3}$ & $\frac{4 \pi}{3}$ & \multirow{2}{*}{$100 \sim 200(5)$} & -1.52070 & +4.40337 & -0.49827 & -7.73447 \\
& & & $-3.17340 \mathfrak{n}_{X_{1}}$ & $+10.53032 \mathfrak{n}_{X_{1}}$ & $-0.05617 \mathfrak{n}_{X_{1}}$ & $-18.79411 \mathfrak{n}_{X_{1}}$ \\
& & & $-0.02594 \mathrm{t}$ & $-0.06618 \mathrm{t}$ & $-0.00672 \mathrm{t}$ & $+0.09834 \mathrm{t}$ \\
\hline \hline
\end{tabular}


The index Eq. (4.22) and $\operatorname{Re} \log Z$ can be computed using the numerical solutions. For simplicity we set $\mathfrak{n}_{q_{j}}=$ $\mathfrak{n}_{q}$ and $\tilde{\mathfrak{n}}_{q_{j}}=\tilde{\mathfrak{n}}_{q}$ for all $j$. Under the similar decomposition

$$
\operatorname{Re} \log Z=A+B_{1} \mathfrak{n}_{X_{1}}+B_{2} \mathfrak{n}_{q}+B_{3} \mathfrak{t},
$$

where we have used the marginality condition on the superpotential $\mathfrak{n}_{X_{1}}+\mathfrak{n}_{X_{2}}=4 / 3, \mathfrak{n}_{X_{3}}=2 / 3$ and $\mathfrak{n}_{q}+\tilde{\mathfrak{n}}_{q}=2 / 3$. Then we perform a linear least-squares fit for $A$ and $B_{a}$ to the function

$$
\begin{aligned}
f(N)= & f_{1} N^{3 / 2}+f_{2} N^{1 / 2}+f_{3} \log N+f_{4} \\
& +\sum_{p=1}^{p_{c}} f_{p+4} N^{(1-2 p) / 2} .
\end{aligned}
$$

The results of the numerical fit for $\operatorname{Re} \log Z$ with $N$ are presented in Table IV. The analytical leading term computed by the index theorem in [26] and the numerical leading term $f_{1} N^{3 / 2}$ match to number of significant digits present in the table. And the numerical results are also independent of $\left\{\Delta_{q}, \tilde{\Delta}_{q}\right\}$. The numerical results indicate that the coefficient $f_{3}$ of the $\log N$ term is precisely $-1 / 2$.

Furthermore, from the numerical results it is shown that by taking

$$
\begin{aligned}
& \Delta_{X_{1}}=\Delta_{3}, \Delta_{m}=k\left(\frac{2 \pi}{3}-\Delta_{4}\right), \\
& \mathfrak{n}_{X_{1}}=\mathfrak{n}_{3}, \mathfrak{t}=-k\left[\frac{2}{3}(1-g)-\mathfrak{n}_{4}\right],
\end{aligned}
$$

where $g$ is the genus and $g=0$ for this case, Model I matches exactly with Model II for every term, including to logarithmic terms which are equal as they are independent of the chemical potentials of the magnetic charges.

\section{THE TOPOLOGICALLY TWISTED INDEX OF $Q^{1,1,1}$}

The field theory dual to M-theory of $\mathrm{AdS}_{4} \times Q^{1,1,1} / \mathbb{Z}_{k}$ was originally discussed in [43,47], see also [48]. The quiver diagram is a particular flavored type of the ABJM quiver:

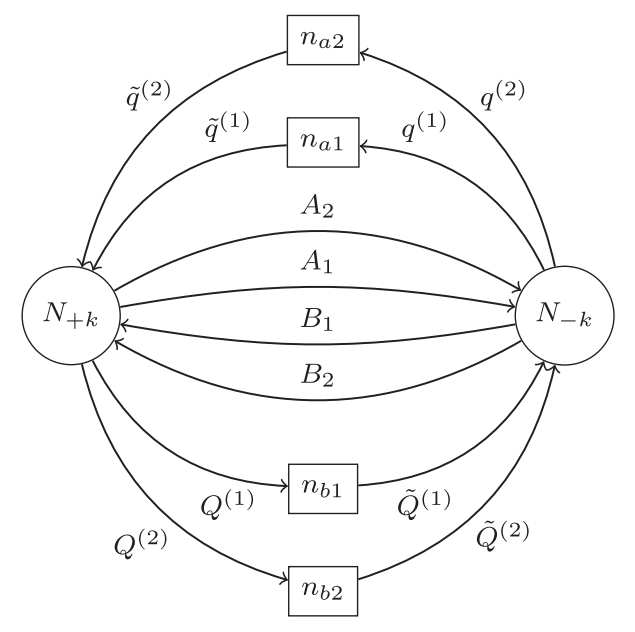

with the superpotential

$$
\begin{aligned}
W= & \operatorname{Tr}\left(A_{1} B_{1} A_{2} B_{2}-A_{1} B_{2} A_{2} B_{1}\right) \\
& +\operatorname{Tr}\left[\sum_{j=1}^{n_{a 1}} q_{j}^{(1)} A_{1} \tilde{q}_{j}^{(1)}+\sum_{j=1}^{n_{a 2}} q_{j}^{(2)} A_{2} \tilde{q}_{j}^{(2)}+\sum_{j=1}^{n_{b 1}} Q_{j}^{(1)} B_{1} \tilde{Q}_{j}^{(1)}+\sum_{j=1}^{n_{b 2}} Q_{j}^{(2)} B_{2} \tilde{Q}_{j}^{(2)}\right] .
\end{aligned}
$$

The free energy of the field theory on $S^{3}$ was shown to match the gravity side in [44,46]. Aspects of the superconformal index have been discussed in [39,40]. The large $N$ analysis of the topologically twisted index was presented in [26] whose notation and leading order analysis we follow very closely.

\section{A. Numerical solutions to the system of BAEs}

From the ingredients of the quiver diagram (5.1) one constructs the topologically twisted index as

$$
\begin{aligned}
Z= & \frac{1}{(N !)^{2}} \sum_{\mathfrak{m}, \tilde{\mathfrak{m}} \in \mathbb{Z}^{N}} \int_{\mathcal{C}} \prod_{i=1}^{N} \frac{d x_{i}}{2 \pi i x_{i}} \frac{d \tilde{x}_{i}}{2 \pi i \tilde{x}_{i}} x_{i}^{\mathrm{t}} \tilde{x}_{i}^{\tilde{\mathfrak{t}}} \xi^{\mathfrak{m}_{i}} \tilde{\xi}^{-\tilde{\mathfrak{m}}_{i}} \times \prod_{i \neq j}^{N}\left(1-\frac{x_{i}}{x_{j}}\right)\left(1-\frac{\tilde{x}_{i}}{\tilde{x}_{j}}\right) \\
& \times \prod_{i, j=1}^{N} \prod_{a=1,2}\left(\frac{\sqrt{\frac{x_{i}}{\tilde{x}_{j}} y_{a}}}{1-\frac{x_{i}}{\tilde{x}_{j}} y_{a}}\right)^{\mathfrak{m}_{i}-\tilde{\mathfrak{m}}_{j}-\mathfrak{n}_{a}+1} \prod_{b=3,4}\left(\frac{\sqrt{\frac{\tilde{x}_{j}}{x_{i}} y_{b}}}{1-\frac{\tilde{x}_{j}}{x_{i}} y_{b}}\right)^{\tilde{\mathfrak{m}}_{j}-\mathfrak{m}_{i}-\mathfrak{n}_{b}+1} \\
& \times \prod_{i=1}^{N} \prod_{k=1,2}\left(\frac{\sqrt{\frac{1}{x_{i}} \tilde{y}_{a k}}}{1-\frac{1}{x_{i}} \tilde{y}_{a k}}\right)^{n\left(-\mathfrak{m}_{i}-\tilde{\mathfrak{n}}_{a k}+1\right)} \times \prod_{j=1}^{N} \prod_{k=1,2}\left(\frac{\sqrt{\tilde{x}_{j} y_{a k}}}{1-\tilde{x}_{j} y_{a k}}\right)^{n\left(\tilde{\mathfrak{m}}_{j}-\mathfrak{n}_{a k}+1\right)} .
\end{aligned}
$$


Performing the summation over magnetic fluxes introducing a large cutoff $M$.

$$
\begin{aligned}
Z= & \frac{1}{(N !)^{2}} \sum_{\mathfrak{m}, \tilde{\mathfrak{m}} \in \mathbb{Z}^{N}} \int_{\mathcal{C}} \prod_{i=1}^{N} \frac{d x_{i}}{2 \pi i x_{i}} \frac{d \tilde{x}_{i}}{2 \pi i \tilde{x}_{i}} x_{i}^{\mathrm{t}} \tilde{x}_{i}^{\tilde{\mathrm{f}}} \times \prod_{i \neq j}^{N}\left(1-\frac{x_{i}}{x_{j}}\right)\left(1-\frac{\tilde{x}_{i}}{\tilde{x}_{j}}\right) \\
& \times \prod_{i, j=1}^{N} \prod_{a=1,2}\left(\frac{\sqrt{\frac{x_{i}}{\tilde{x}_{j}} y_{a}}}{1-\frac{x_{i}}{\tilde{x}_{j}} y_{a}}\right)^{1-\mathfrak{n}_{a}} \prod_{b=3,4}\left(\frac{\sqrt{\frac{\tilde{x}_{j}}{x_{i}} y_{b}}}{1-\frac{\tilde{x}_{j}}{x_{i}} y_{b}}\right)^{1-\mathfrak{n}_{b}} \\
& \times \prod_{i=1}^{N} \prod_{k=1,2}\left(\frac{\sqrt{\frac{1}{x_{i}} \tilde{y}_{a k}}}{1-\frac{1}{x_{i}} \tilde{y}_{a k}}\right)^{n\left(1-\tilde{\mathfrak{n}}_{a k}\right)} \\
& \times \prod_{j=1}^{N} \prod_{k=1,2}\left(\frac{\sqrt{\tilde{x}_{j} y_{a k}}}{1-\tilde{x}_{j} y_{a k}}\right)^{n\left(1-\mathfrak{n}_{a k}\right)} \prod_{i=1}^{N} \frac{\left(e^{i B_{i}}\right)^{M}}{e^{i B_{i}}-1} \prod_{j=1}^{N} \frac{\left(e^{i \tilde{B}_{j}}\right)^{M}}{e^{i \tilde{B}_{j}}-1},
\end{aligned}
$$

where the Bethe Ansatz equations are

$$
\begin{aligned}
& 1=e^{i B_{i}}=\xi \prod_{j=1}^{N} \frac{\left(1-y_{3} \frac{\tilde{x}_{j}}{x_{i}}\right)\left(1-y_{4} \frac{\tilde{x}_{j}}{x_{i}}\right)}{\left(1-y_{1}^{-1} \frac{\tilde{x}_{j}}{x_{i}}\right)\left(1-y_{2}^{-1} \frac{\tilde{x}_{j}}{x_{i}}\right)} \times \prod_{k=1,2}\left(\frac{\sqrt{\frac{1}{x_{i}} \tilde{y}_{a k}}}{1-\frac{1}{x_{i}} \tilde{y}_{a k}}\right)^{-n}, \\
& 1=e^{i \tilde{B}_{j}}=\tilde{\xi} \prod_{i=1}^{N} \frac{\left(1-y_{3} \frac{\tilde{x}_{j}}{x_{i}}\right)\left(1-y_{4} \frac{\tilde{x}_{j}}{x_{i}}\right)}{\left(1-y_{1}^{-1} \frac{\tilde{x}_{j}}{x_{i}}\right)\left(1-y_{2}^{-1} \frac{\tilde{x}_{j}}{x_{i}}\right)} \times \prod_{k=1,2}\left(\frac{\sqrt{\tilde{x}_{j} y_{a k}}}{1-\tilde{x}_{j} y_{a k}}\right)^{-n} .
\end{aligned}
$$

The compact expression is

$$
\begin{aligned}
Z\left(y_{a}, \mathfrak{n}_{a}\right)= & \prod_{k=1,2}\left[\tilde{y}_{a k^{\frac{1}{2}} N n\left(1-\tilde{\mathfrak{n}}_{a k}\right)} y_{a k}^{\frac{1}{2} N n\left(1-\mathfrak{n}_{a k}\right)}\right] \times \prod_{a=1}^{4} y_{a}^{-\frac{1}{2} N^{2} \mathfrak{n}_{a}} \\
& \times \sum_{I \in B A E}\left[\frac{1}{\operatorname{det} \mathbb{B}} \frac{\prod_{i=1}^{N} x_{i}^{N+\mathfrak{t}} \tilde{x}_{i}^{N+\tilde{\mathfrak{t}}} \prod_{i \neq j}\left(1-\frac{x_{i}}{x_{j}}\right)\left(1-\frac{\tilde{x}_{i}}{\tilde{x}_{j}}\right)}{\prod_{i, j=1}^{N} \prod_{a=1,2}\left(\tilde{x}_{j}-y_{a} x_{i}\right)^{1-\mathfrak{n}_{a}} \prod_{a=3,4}\left(x_{i}-y_{a} \tilde{x}_{j}\right)^{1-\mathfrak{n}_{a}}}\right. \\
& \left.\times \prod_{i=1}^{N} \prod_{k=1,2} \frac{x_{i}^{\frac{1}{2} n\left(1-\tilde{\mathfrak{n}}_{a k}\right)} \tilde{x}_{i}^{\frac{1}{2} n\left(1-\mathfrak{n}_{a k}\right)}}{\left(x_{i}-\tilde{y}_{a k}\right)^{n\left(1-\tilde{\mathfrak{n}}_{a k}\right)}\left(1-\tilde{x}_{i} y_{a k}\right)^{n\left(1-\mathfrak{n}_{a k}\right)}}\right]
\end{aligned}
$$

The matrix $\mathbb{B}$ is

$$
\left.\mathbb{B}\right|_{\mathrm{BAEs}}=\left(\begin{array}{cc}
\delta_{j l}\left[-\sum_{m=1}^{N} G_{j m}+\sum_{k=1,2} \frac{n x_{j}}{x_{j}-\tilde{y}_{a k}}-n\right] & G_{j l} \\
-G_{l j} & \delta_{j l}\left[\sum_{m=1}^{N} G_{m j}+\sum_{k=1,2} \frac{n \tilde{x}_{j}}{\tilde{x}_{j}-y_{a k}^{-1}}-n\right]
\end{array}\right) .
$$


The Bethe potential is

$$
\begin{aligned}
\mathcal{V}= & \sum_{i=1}^{N}\left[-\Delta_{m}^{(1)} u_{i}+\Delta_{m}^{(2)} \tilde{u}_{i}-2 \pi\left(\tilde{n}_{i} \tilde{u}_{i}-n_{i} u_{i}\right)\right]+\sum_{i, j=1}^{N}\left[\sum_{a=3,4} \operatorname{Li}_{2}\left(e^{i\left(\tilde{u}_{j}-u_{i}+\Delta_{a}\right)}\right)-\sum_{a=1,2} \operatorname{Li}_{2}\left(e^{i\left(\tilde{u}_{j}-u_{i}-\Delta_{a}\right)}\right)\right] \\
& +n \sum_{i=1}^{N} \sum_{k=1,2}\left[\operatorname{Li}_{2}\left(e^{i\left(-u_{i}+\tilde{\Delta}_{a k}\right)}\right)-\operatorname{Li}_{2}\left(e^{i\left(-\tilde{u}_{i}-\Delta_{a k}\right)}\right)\right] \\
& +\frac{n}{2} \sum_{i=1}^{N} \sum_{k=1,2}\left[\left(\tilde{\Delta}_{a k}-\pi\right) u_{i}+\left(\Delta_{a k}-\pi\right) \tilde{u}_{i}\right]-\frac{n}{2} \sum_{i=1}^{N}\left[u_{i}^{2}-\tilde{u}_{i}^{2}\right],
\end{aligned}
$$

where

$$
\sum_{i=1}^{N}\left[-2 \pi\left(\tilde{n}_{i} \tilde{u}_{i}-n_{i} u_{i}\right)\right]=\left(4 \pi-\sum_{a=1}^{4} \Delta_{a}\right) \sum_{i>j}^{N}\left(\tilde{u}_{j}-u_{i}\right)=2 \pi \sum_{i>j}^{N}\left(\tilde{u}_{j}-u_{i}\right)
$$

It is assumed that $0<-v(t)+\tilde{\Delta}_{a 1}<2 \pi, 0<-v(t)+$ $\tilde{\Delta}_{a 2}<2 \pi, 0<\tilde{v}(t)+\Delta_{a 1}<2 \pi$ and $0<\tilde{v}(t)+\Delta_{a 2}<2 \pi$. Using $\Delta_{1}=\Delta_{2}=\pi-\Delta_{3}=\pi-\Delta_{4}=\Delta$ and the marginality condition from the superpotential $\Delta_{1}+\Delta_{a 1}+$ $\tilde{\Delta}_{a 1}=2 \pi, \Delta_{2}+\Delta_{a 2}+\tilde{\Delta}_{a 2}=2 \pi$ we get

$$
\frac{v(t)+\tilde{v}(t)}{2} \in \begin{cases}\left(\frac{-\Delta-2 \Delta_{a 1}}{2}, \frac{+\Delta+2 \tilde{\Delta}_{a 2}}{2}\right), & \Delta_{a 1}<\Delta_{a 2} \\ \left(\frac{-\Delta-2 \Delta_{a 2}}{2}, \frac{+\Delta+2 \tilde{\Delta}_{a 1}}{2}\right), & \Delta_{a 1} \geq \Delta_{a 2}\end{cases}
$$

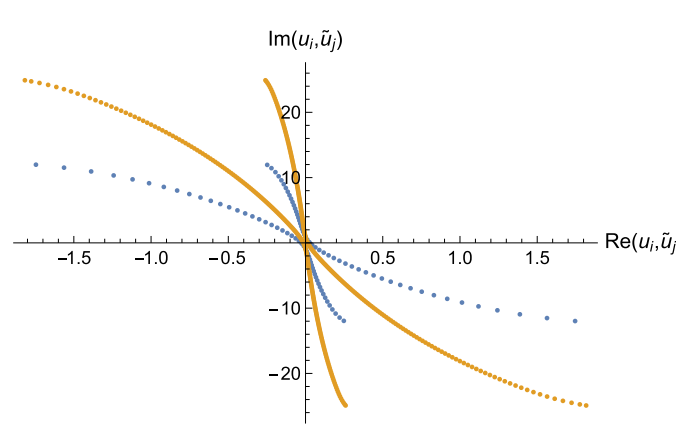

(a) Eigenvalue distribution

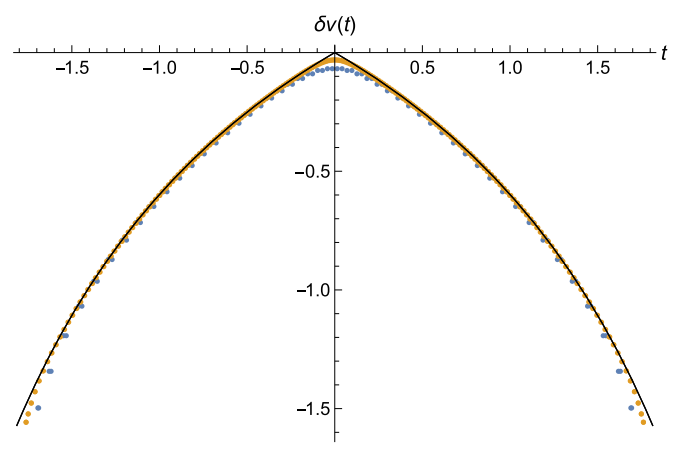

(c) Real part difference $\delta v(t)$

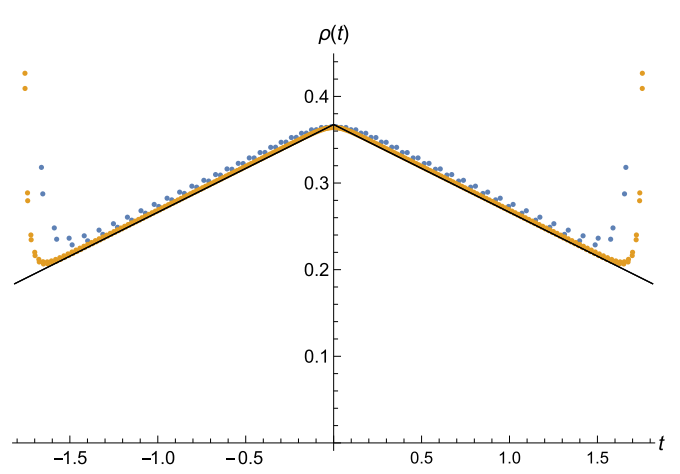

(b) Eigenvalue density $\rho(t)$

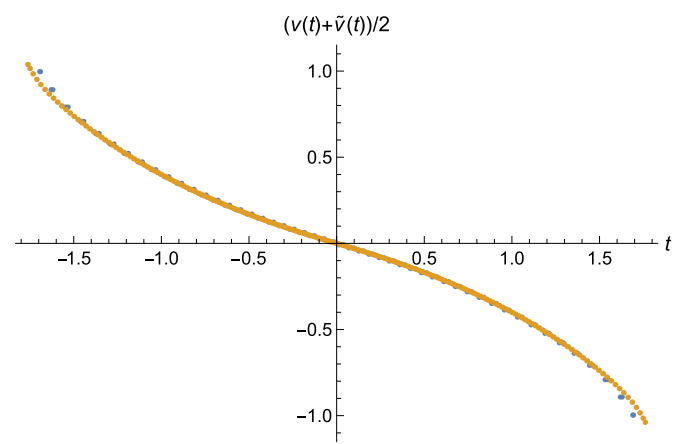

(d) Real part axis $(v(t)+\tilde{v}(t)) / 2$

$$
\begin{aligned}
& N=50, \Delta=\frac{\pi}{2}, \\
& \left\{\Delta_{m}^{(1)}, \Delta_{m}^{(2)}\right\}=\{0,0\}, \\
& \Delta_{m}=0, n=1, \\
& \left\{\Delta_{a 1}, \Delta_{a 2}\right\}=\left\{\frac{3 \pi}{4}, \frac{3 \pi}{4}\right\} \\
& N=200, \Delta=\frac{\pi}{2}, \\
& \left\{\Delta_{m}^{(1)}, \Delta_{m}^{(2)}\right\}=\{0,0\}, \\
& \Delta_{m}=0, n=1, \\
& \left\{\Delta_{a 1}, \Delta_{a 2}\right\}=\left\{\frac{3 \pi}{4}, \frac{3 \pi}{4}\right\}
\end{aligned}
$$

FIG. 13. Eigenvalues for $\Delta_{a}=\left\{\frac{\pi}{2}, \frac{\pi}{2}, \frac{\pi}{2}, \frac{\pi}{2}\right\}$ for $N=50$ (blue) and 200 (orange) with the same other parameters. 


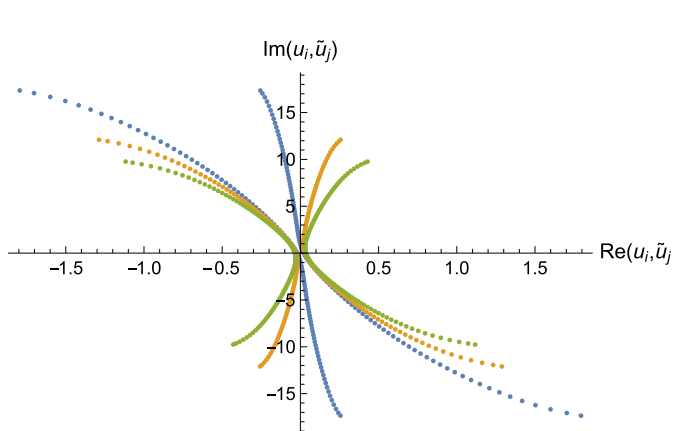

(a) Eigenvalue distribution

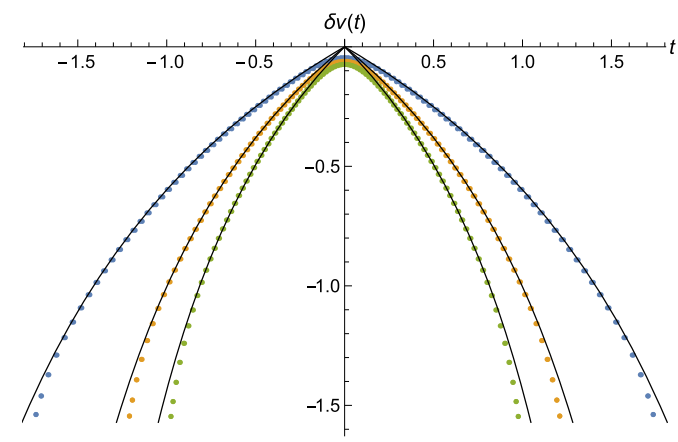

(c) Real part difference $\delta v(t)$

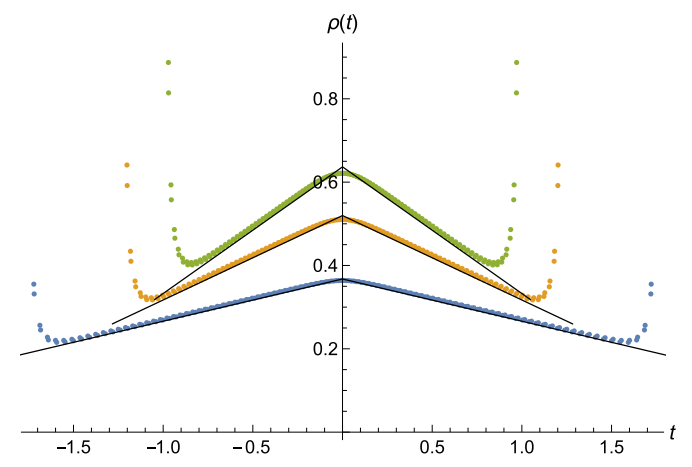

(b) Eigenvalue density $\rho(t)$

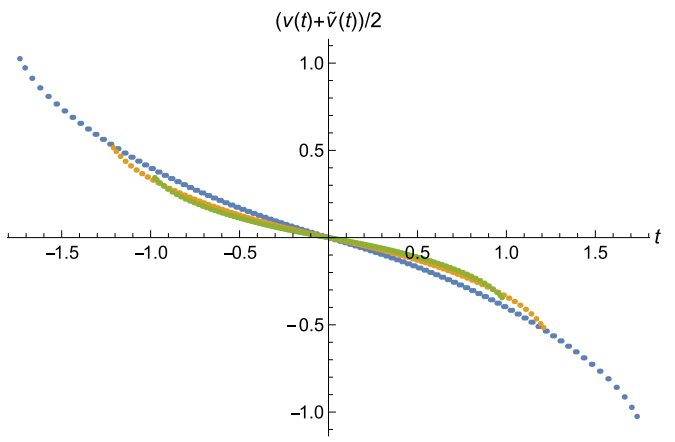

(d) Real part axis $(v(t)+\tilde{v}(t)) / 2$

$$
\begin{aligned}
& N=100, \Delta=\frac{\pi}{2}, \\
& \left\{\Delta_{m}^{(1)}, \Delta_{m}^{(2)}\right\}=\{0,0\}, \\
& \Delta_{m}=0, n=1 \\
& \left\{\Delta_{a 1}, \Delta_{a 2}\right\}=\left\{\frac{3 \pi}{4}, \frac{3 \pi}{4}\right\} \\
& N=100, \Delta=\frac{\pi}{2} \\
& \\
& \left\{\Delta_{m}^{(1)}, \Delta_{m}^{(2)}\right\}=\{0,0\} \\
& \Delta_{m}=0, n=2 \\
& \left\{\Delta_{a 1}, \Delta_{a 2}\right\}=\left\{\frac{3 \pi}{4}, \frac{3 \pi}{4}\right\} \\
& N=100, \Delta=\frac{\pi}{2}, \\
& \\
& \left.N \Delta_{m}^{(1)}, \Delta_{m}^{(2)}\right\}=\{0,0\} \\
& \Delta_{m}=0, n=3 \\
& \left\{\Delta_{a 1}, \Delta_{a 2}\right\}=\left\{\frac{3 \pi}{4}, \frac{3 \pi}{4}\right\}
\end{aligned}
$$

FIG. 14. Eigenvalues for $\Delta_{a}=\left\{\frac{\pi}{2}, \frac{\pi}{2}, \frac{\pi}{2}, \frac{\pi}{2}\right\}$ for $n=1$ (blue), 2 (orange) and 3 (green) with the same other parameters.

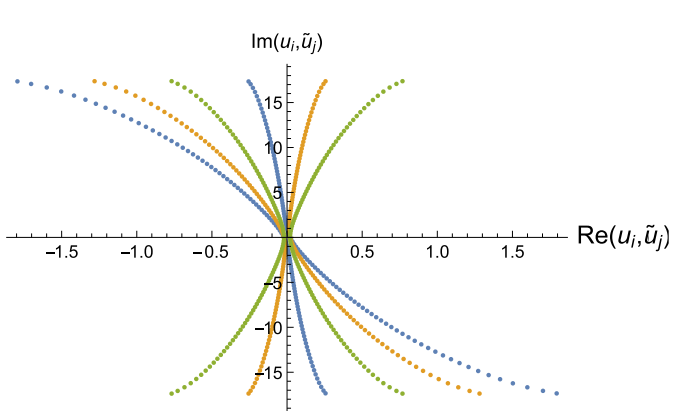

(a) Eigenvalue distribution

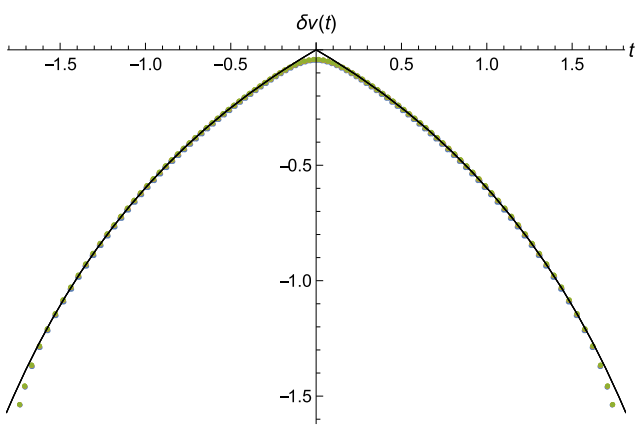

(c) Real part difference $\delta v(t)$

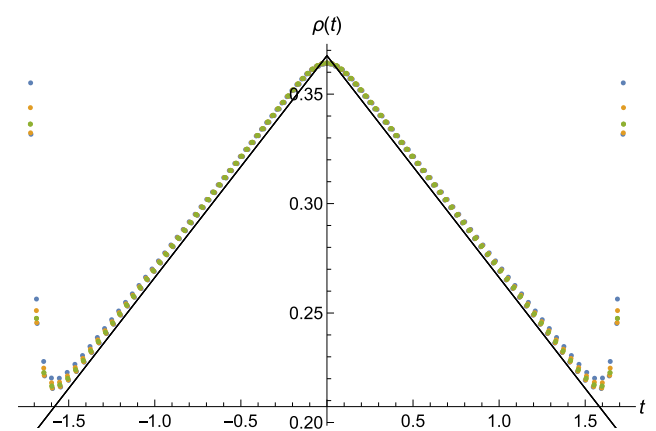

(b) Eigenvalue density $\rho(t)$

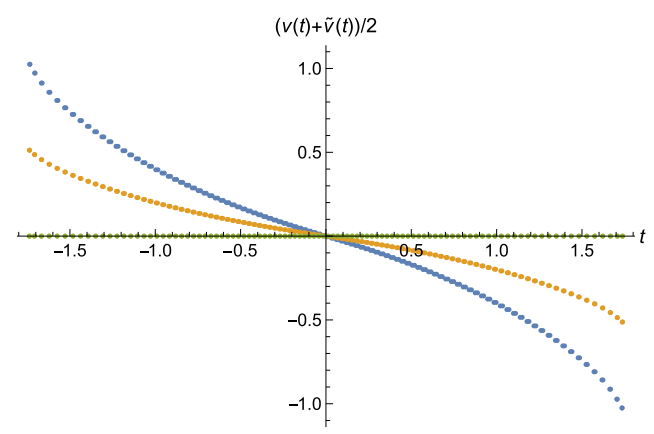

(d) Real part axis $(v(t)+\tilde{v}(t)) / 2$
$N=100, \Delta=\frac{\pi}{2}$, $\left\{\Delta_{m}^{(1)}, \Delta_{m}^{(2)}\right\}=\{0,0\}$,

$\Delta_{m}=0, n=1$,

$\left\{\Delta_{a 1}, \Delta_{a 2}\right\}=\left\{\frac{3 \pi}{4}, \frac{3 \pi}{4}\right\}$.

$N=100, \Delta=\frac{\pi}{2}$,

$\left\{\Delta_{m}^{(1)}, \Delta_{m}^{(2)}\right\}=\left\{\frac{\pi}{2}, \frac{\pi}{2}\right\}$,

$\Delta_{m}=0, n=1$,

$\left\{\Delta_{a 1}, \Delta_{a 2}\right\}=\left\{\frac{3 \pi}{4}, \frac{3 \pi}{4}\right\}$.

$N=100, \Delta=\frac{\pi}{2}$,

$\left\{\Delta_{m}^{(1)}, \Delta_{m}^{(2)}\right\}=\{\pi, \pi\}$,

$\Delta_{m}=0, n=1$,

$\left\{\Delta_{a 1}, \Delta_{a 2}\right\}=\left\{\frac{3 \pi}{4}, \frac{3 \pi}{4}\right\}$.

FIG. 15. Eigenvalues for $\Delta_{a}=\left\{\frac{\pi}{2}, \frac{\pi}{2}, \frac{\pi}{2}, \frac{\pi}{2}\right\}$ for $\left\{\Delta_{m}^{(1)}, \Delta_{m}^{(2)}\right\}=\{0,0\}$ (blue), $\left\{\frac{\pi}{2}, \frac{\pi}{2}\right\}$ (orange) and $\{\pi, \pi\}$ (green) keeping the same $\Delta_{m}=0$ with the same other parameters. 


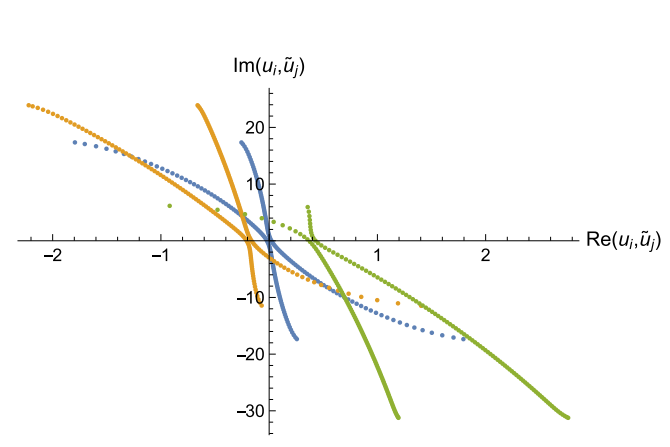

(a) Eigenvalue distribution

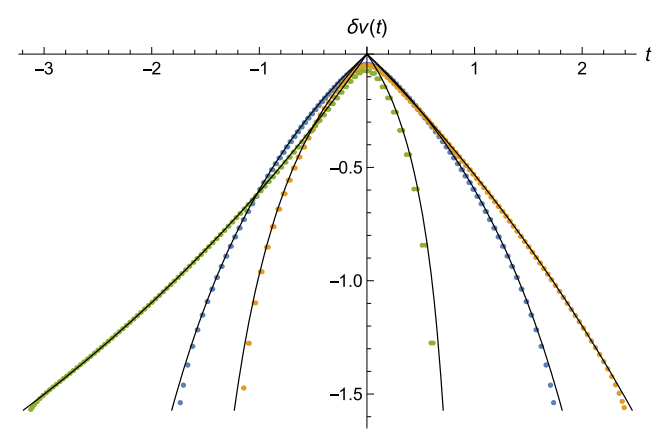

(c) Real part difference $\delta v(t)$

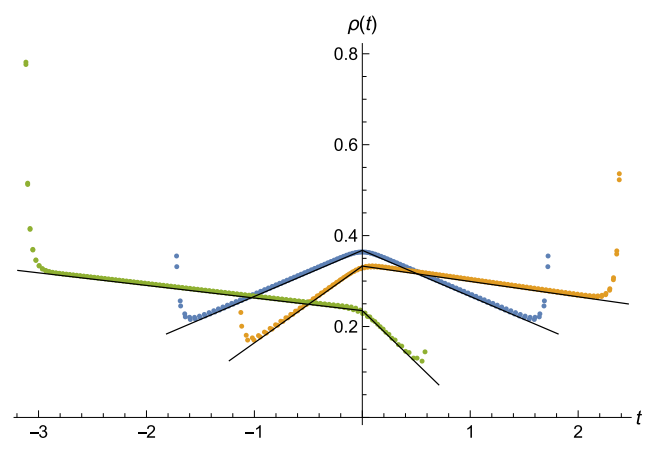

(b) Eigenvalue density $\rho(t)$

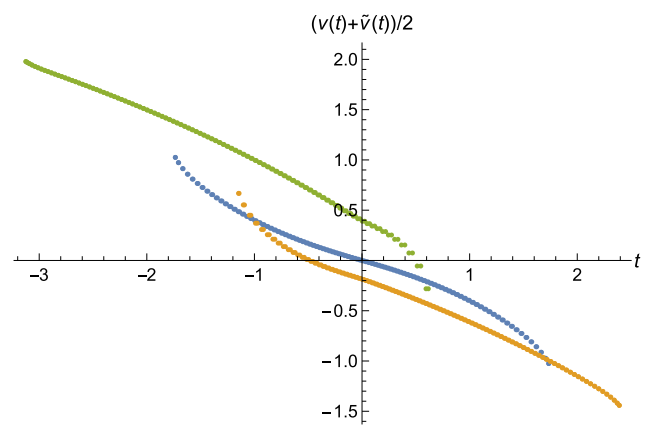

(d) Real part axis $(v(t)+\tilde{v}(t)) / 2$
$N=100, \Delta=\frac{\pi}{2}$

$\left\{\Delta_{m}^{(1)}, \Delta_{m}^{(2)}\right\}=\{0,0\}$,

$\Delta_{m}=0, n=1$,

$\left\{\Delta_{a 1}, \Delta_{a 2}\right\}=\left\{\frac{3 \pi}{4}, \frac{3 \pi}{4}\right\}$.

$N=100, \Delta=\frac{\pi}{2}$,

$\left\{\Delta_{m}^{(1)}, \Delta_{m}^{(2)}\right\}=\left\{\frac{\pi}{6},-\frac{\pi}{6}\right\}$,

$\Delta_{m}=-\frac{\pi}{3}, n=1$,

$\left\{\Delta_{a 1}, \Delta_{a 2}\right\}=\left\{\frac{3 \pi}{4}, \frac{3 \pi}{4}\right\}$.

$N=100, \Delta=\frac{\pi}{2}$,

$\left\{\Delta_{m}^{(1)}, \Delta_{m}^{(2)}\right\}=\{-1,1\}$,

$\Delta_{m}=2, n=1$,

$\left\{\Delta_{a 1}, \Delta_{a 2}\right\}=\left\{\frac{3 \pi}{4}, \frac{3 \pi}{4}\right\}$.

FIG. 16. Eigenvalues for $\Delta_{a}=\left\{\frac{\pi}{2}, \frac{\pi}{2}, \frac{\pi}{2}, \frac{\pi}{2}\right\}$ for $\Delta_{m}=0,\left\{\Delta_{m}^{(1)}, \Delta_{m}^{(2)}\right\}=\{0,0\}$ (blue), $\Delta_{m}=-\frac{\pi}{3},\left\{\Delta_{m}^{(1)}, \Delta_{m}^{(2)}\right\}=\left\{-\frac{\pi}{6}, \frac{\pi}{6}\right\}$ (orange) and $\Delta_{m}=2,\left\{\Delta_{m}^{(1)}, \Delta_{m}^{(2)}\right\}=\{1,-1\}$ (green) with the same other parameters.

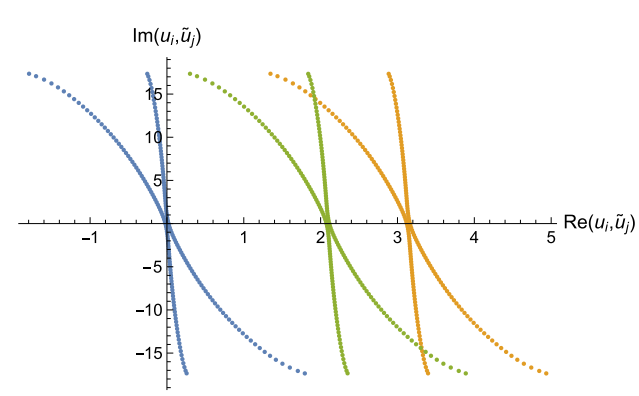

(a) Eigenvalue distribution

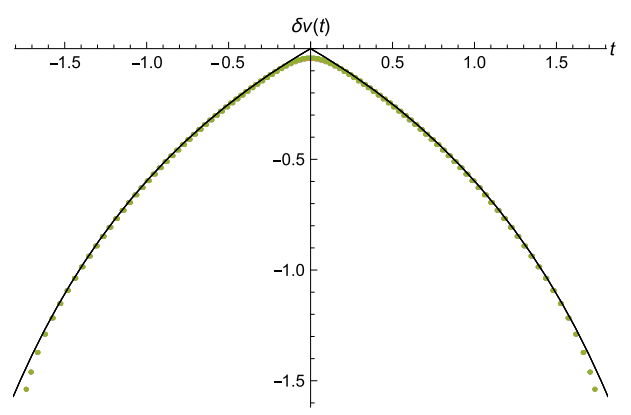

(c) Real part difference $\delta v(t)$

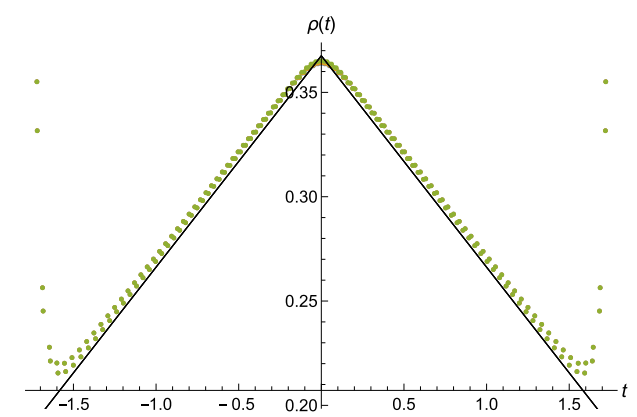

(b) Eigenvalue density $\rho(t)$

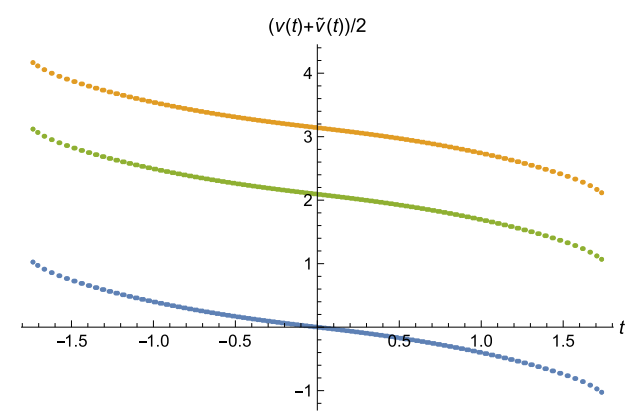

(d) Real part axis $(v(t)+\tilde{v}(t)) / 2$
$N=100, \Delta=\frac{\pi}{2}$,

$\left\{\Delta_{m}^{(1)}, \Delta_{m}^{(2)}\right\}=\{0,0\}$,

$\Delta_{m}=0, n=1$,

$\left\{\Delta_{\mathrm{a} 1}, \Delta_{\mathrm{a} 2}\right\}=\left\{\frac{3 \pi}{4}, \frac{3 \pi}{4}\right\}$.

$N=100, \Delta=\frac{\pi}{2}$,

$\left\{\Delta_{m}^{(1)}, \Delta_{m}^{(2)}\right\}=\{0,0\}$,

$\Delta_{m}=0, n=1$,

$\left\{\Delta_{a 1}, \Delta_{a 2}\right\}=\left\{-\frac{\pi}{3},-\frac{\pi}{6}\right\}$.

$N=100, \Delta=\frac{\pi}{2}$,

$\left\{\Delta_{m}^{(1)}, \Delta_{m}^{(2)}\right\}=\{0,0\}$,

$\Delta_{m}=0, n=1$,

$\left\{\Delta_{a 1}, \Delta_{a 2}\right\}=\left\{\frac{\pi}{4},-\frac{\pi}{12}\right\}$.

FIG. 17. Eigenvalues for $\Delta_{a}=\left\{\frac{\pi}{2}, \frac{\pi}{2}, \frac{\pi}{2}, \frac{\pi}{2}\right\}$ for $\left\{\Delta_{a 1}, \Delta_{a 2}, \tilde{\Delta}_{a 1}, \tilde{\Delta}_{a 2}\right\}=\left\{\frac{3 \pi}{4}, \frac{3 \pi}{4}, \frac{3 \pi}{4}, \frac{3 \pi}{4}\right\}$ (blue), $\left\{-\frac{\pi}{3},-\frac{\pi}{6}, \frac{11 \pi}{6}, \frac{5 \pi}{3}\right\}$ (orange) and $\left\{\frac{\pi}{4},-\frac{\pi}{12}, \frac{5 \pi}{4}, \frac{19 \pi}{12}\right\}$ (green) with the same other parameters. 


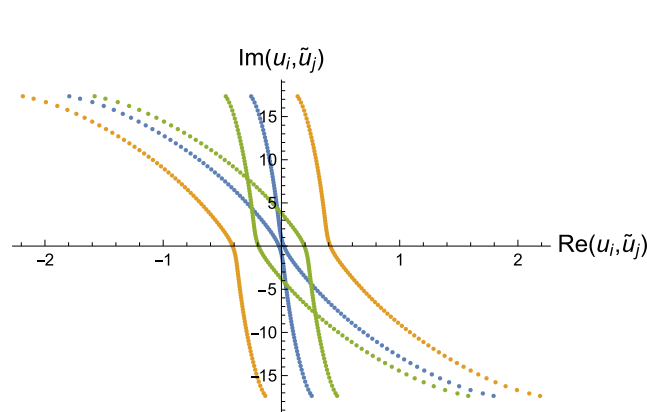

(a) Eigenvalue distribution

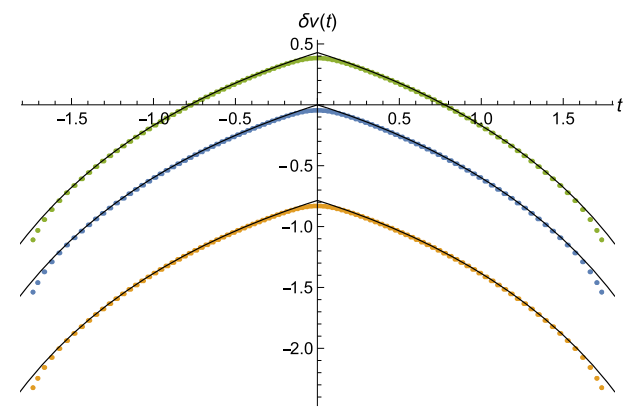

(c) Real part difference $\delta v(t)$

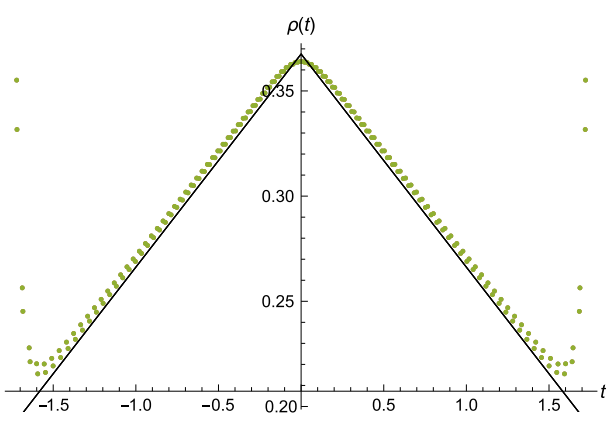

(b) Eigenvalue density $\rho(t)$

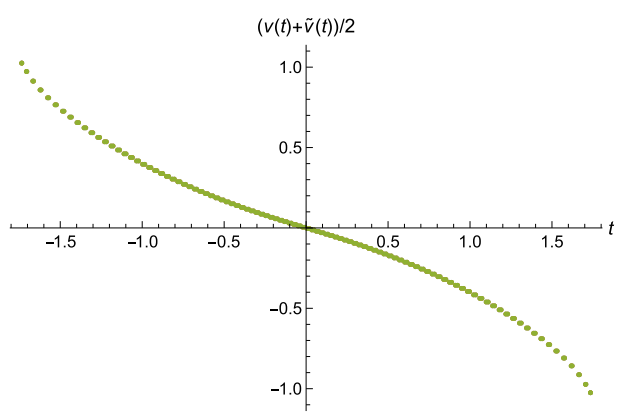

(d) Real part axis $(v(t)+\tilde{v}(t)) / 2$
$N=100, \Delta=\frac{\pi}{2}$, $\left\{\Delta_{m}^{(1)}, \Delta_{m}^{(2)}\right\}=\{0,0\}$, $\Delta_{m}=0, n=1$, $\left\{\Delta_{a 1}, \Delta_{a 2}\right\}$ $=\left\{\frac{3 \pi}{4}, \frac{3 \pi}{4}\right\}$.

$N=100, \Delta=\frac{\pi}{4}$, $\left\{\Delta_{m}^{(1)}, \Delta_{m}^{(2)}\right\}=\{0,0\}$, $\Delta_{m}=0, n=1$, $\left\{\Delta_{a 1}, \Delta_{a 2}\right\}$ $=\left\{\frac{7 \pi}{8}, \frac{7 \pi}{8}\right\}$.

$N=100, \Delta=2$, $\left\{\Delta_{m}^{(1)}, \Delta_{m}^{(2)}\right\}=\{0,0\}$, $\Delta_{m}=0, n=1$, $\left\{\Delta_{a 1}, \Delta_{a 2}\right\}$ $=\{-1+\pi,-1+\pi\}$.

FIG. 18. Eigenvalues for $\Delta_{a}=\left\{\frac{\pi}{2}, \frac{\pi}{2}, \frac{\pi}{2}, \frac{\pi}{2}\right\},\left\{\Delta_{a 1}, \Delta_{a 2}, \tilde{\Delta}_{a 1}, \tilde{\Delta}_{a 2}\right\}=\left\{\frac{3 \pi}{4}, \frac{3 \pi}{4}, \frac{3 \pi}{4}, \frac{3 \pi}{4}\right\} \quad$ (blue), $\Delta_{a}=\left\{\frac{\pi}{4}, \frac{\pi}{4}, \frac{3 \pi}{4}, \frac{3 \pi}{4}\right\},\left\{\Delta_{a 1}, \Delta_{a 2}, \tilde{\Delta}_{a 1}, \tilde{\Delta} a 2\right\}=$ $\left\{\frac{7 \pi}{8}, \frac{7 \pi}{8}, \frac{7 \pi}{8}, \frac{7 \pi}{8}\right\}$ (orange) and $\Delta_{a}=\{2,2, \pi-2, \pi-2\},\left\{\Delta_{a 1}, \Delta_{a 2}, \tilde{\Delta}_{a 1}, \tilde{\Delta}_{a 2}\right\}=\{\pi-1, \pi-1, \pi-1, \pi-1\}$ (green) with the same other parameters.

Thus we set the initial real part axis to be

$$
\frac{v(t)+\tilde{v}(t)}{2}=\frac{2 \pi-\Delta-\Delta_{a 1}-\Delta_{a 2}}{2}
$$

Note that we have specialized from the general flavored ABJM quiver (5.1) to the particular case corresponding to the dual of $\mathrm{AdS}_{4} \times Q^{1,1,1} / \mathbb{Z}_{n}$ which in the notation we have introduced implies: $k=0, n_{a 1}=n_{a 2}=n, n_{b 1}=$ $n_{b 2}=0$ where the subindex $b i$ corresponds to the fields $Q$ and $\tilde{Q}$. Our goal is thus, to explore the numerical behavior of the index as a function of $N$ and $\Delta_{a}$ just as in the ABJM case but also as functions of $\left\{\Delta_{m}^{(1)}, \Delta_{m}^{(2)}\right\}, n$ and $\left\{\Delta_{a 1}, \Delta_{a 2}, \tilde{\Delta}_{a 1}, \tilde{\Delta}_{a 2}\right\}$.

We set $\Delta_{m}=\Delta_{m}^{(2)}-\Delta_{m}^{(1)}$. The numerical solutions for different values of $N, n,\left\{\Delta_{m}^{(1)}, \Delta_{m}^{(2)}\right\}, \Delta_{m},\left\{\Delta_{a 1}, \Delta_{a 2}\right.$, $\left.\tilde{\Delta}_{a 1}, \tilde{\Delta}_{a 2}\right\}$ and $\Delta_{a}=\left\{\Delta_{1}, \Delta_{2}, \Delta_{3}, \Delta_{4}\right\}(\Delta)$ are shown in Figs. 13-18.

Let us briefly summarize the salient features of the exact eigenvalues that we find. Similar to the index of $N^{0,1,0}$ in section III A, the eigenvalues are not reflectively symmetric about a particular real axis, except for the case that $\left(\Delta_{m}^{(1)}+\Delta_{m}^{(2)}\right) / 2=\pi$. Furthermore, the imaginary part of $u_{i}$ is not exactly the same as $\tilde{u}_{i}$ so that there are two numerical results of the eigenvalue density $\rho(t)$, the real part difference $\delta v(t)$ and the real part axis $(v(t)+\tilde{v}(t)) / 2$. In addition, Figs. 15 and 17 show that the values of $\left\{\Delta_{m}^{(1)}, \Delta_{m}^{(2)}\right\}$ keeping the same $\Delta_{m}$ and the values of $\left\{\Delta_{a 1}, \Delta_{a 2}, \tilde{\Delta}_{a 1}, \tilde{\Delta}_{a 2}\right\}$ have effects only on the real part axis $(v(t)+\tilde{v}(t)) / 2$.

\section{B. The subleading term of the index at large $N$}

The index should take the form

$$
\begin{aligned}
\operatorname{Re} \log Z= & f_{1}(n, \Delta, \mathfrak{n}, \mathfrak{t}) N^{3 / 2}+f_{2}(n, \Delta, \mathfrak{n}, \mathfrak{t}) N^{1 / 2} \\
& +f_{3}(n, \Delta, \mathfrak{n}, \mathfrak{t}) \log N+f_{4}(n, \Delta, \mathfrak{n}, \mathfrak{t}) \\
& +\mathcal{O}\left(N^{-1 / 2}\right),
\end{aligned}
$$

where here $\Delta$ represents all of the chemical potentials as above and the functions $f_{1}, f_{2}, f_{3}$ and $f_{4}$ are linear in the magnetic fluxes $\mathfrak{n}$ and $\mathfrak{t}$.

The index Eq. (5.6) and Re $\log Z$ can be computed using the numerical solutions. Under the similar decomposition

$\operatorname{Re} \log Z=A+B_{1} \mathfrak{n}_{1}+B_{2} \mathfrak{n}_{2}+B_{3} \mathfrak{n}_{3}+B_{4} \mathfrak{n}_{a 1}$

$$
+B_{5} \mathfrak{n}_{a 2}+B_{6} \mathfrak{t}+B_{7} \tilde{\mathfrak{t}},
$$

where we have used the marginality condition on the superpotential $\sum_{a=1}^{4} \mathfrak{n}_{a}=2, \quad \mathfrak{n}_{1}+\mathfrak{n}_{a 1}+\tilde{\mathfrak{n}}_{a 1}=2$ and $\mathfrak{n}_{2}+\mathfrak{n}_{a 2}+\tilde{\mathfrak{n}}_{a 2}=2$. Then we perform a linear leastsquares fit for $A$ and $B_{a}$ to the function 
TABLE V. $\quad\left(Q^{1,1,1}\right)$ Numerical fit for Re $\log Z=f_{1} N^{3 / 2}+f_{2} N^{1 / 2}+f_{3} \log N+f_{4}+\sum_{p=1}^{p_{c}=5} f_{p+4} N^{(1-2 p) / 2}$ and $N$ ranges from 100 to 200 in steps of 5 except for four cases. For numerical stability and accuracy, in the cases $\Delta=\frac{\pi}{2}, \Delta_{m}=2,\left\{\Delta_{m}^{(1)}, \Delta_{m}^{(2)}\right\}=\{-1,+1\}$ and $\left\{\Delta_{m}^{(1)}, \Delta_{m}^{(2)}\right\}=\{\pi-1, \pi+1\}, \quad N$ ranges from 100 to 200 in steps of 5 but $p_{c}=15$. In the case $\Delta=\frac{\pi}{4}, \Delta_{m}=-\frac{3 \pi}{2},\left\{\Delta_{m}^{(1)}, \Delta_{m}^{(2)}\right\}=\left\{\frac{3 \pi}{2}, 0\right\}, n=3, N$ ranges from 100 to 300 in steps of 5 and $p_{c}=30$. And in the case $\Delta=2, \Delta_{m}=\frac{4 \pi}{3},\left\{\Delta_{m}^{(1)}, \Delta_{m}^{(2)}\right\}=\left\{\frac{\pi}{3}, \frac{5 \pi}{3}\right\}, n=2, N$ ranges from 200 to 400 in steps of 5 and $p_{c}=30$.

\begin{tabular}{|c|c|c|c|c|c|c|c|c|c|}
\hline \multicolumn{10}{|c|}{ (a) $k=0 ; \Delta_{a}=\left\{\frac{\pi}{2}, \frac{\pi}{2}, \frac{\pi}{2}, \frac{\pi}{2}\right\}\left(\Delta=\frac{\pi}{2}\right)$} \\
\hline$\Delta_{m}^{(1)}$ & $\Delta_{m}^{(2)}$ & $\Delta_{m}$ & $n$ & $\Delta_{a 1}$ & $\Delta_{a 2}$ & $f_{1}$ & $f_{2}$ & $f_{3}$ & $f_{4}$ \\
\hline 0 & 0 & 0 & 1 & $\frac{3 \pi}{4}$ & $\frac{3 \pi}{4}$ & -2.41840 & $\begin{array}{l}+1.81381 \\
+0.60460 \mathrm{t} \\
-0.60460 \tilde{\mathrm{t}}\end{array}$ & -0.50033 & -2.13933 \\
\hline 0 & 0 & 0 & 2 & $\frac{3 \pi}{4}$ & $\frac{3 \pi}{4}$ & -3.42013 & $\begin{array}{l}+1.28258 \\
+0.42752 \mathrm{t} \\
-0.42752 \tilde{\mathrm{t}}\end{array}$ & -0.50064 & $\begin{array}{l}-2.03420 \\
+0.00004 \mathrm{t} \\
-0.00004 \tilde{\mathrm{t}}\end{array}$ \\
\hline 0 & 0 & 0 & 3 & $\frac{3 \pi}{4}$ & $\frac{3 \pi}{4}$ & -4.18879 & $\begin{array}{l}+1.04723 \\
+0.34907 \mathrm{t} \\
-0.34907 \tilde{\mathrm{t}}\end{array}$ & $\begin{array}{c}-0.50087 \\
-0.00005 \mathrm{t} \\
+0.00005 \tilde{\mathrm{t}}\end{array}$ & $\begin{array}{l}-2.23075 \\
+0.00030 \mathrm{t} \\
-0.00030 \tilde{\mathrm{t}}\end{array}$ \\
\hline$\pi$ & $\pi$ & 0 & 1 & $\frac{3 \pi}{4}$ & $\frac{3 \pi}{4}$ & -2.41840 & +1.81384 & -0.50098 & -2.13483 \\
\hline$\pi$ & $\pi$ & 0 & 2 & $\frac{3 \pi}{4}$ & $\frac{3 \pi}{4}$ & -3.42013 & +1.28259 & -0.50097 & -2.03192 \\
\hline$\pi$ & $\pi$ & 0 & 3 & $\frac{3 \pi}{4}$ & $\frac{3 \pi}{4}$ & -4.18879 & +1.04725 & -0.50117 & -2.22872 \\
\hline$\frac{\pi}{2}$ & $\frac{\pi}{2}$ & 0 & 1 & $\frac{3 \pi}{4}$ & $\frac{3 \pi}{4}$ & -2.41840 & $\begin{array}{c}+1.81383 \\
+0.30230 \mathrm{t} \\
-0.30230 \tilde{\mathrm{t}}\end{array}$ & -0.50082 & -2.13596 \\
\hline$\frac{\pi}{6}$ & $-\frac{\pi}{6}$ & $-\frac{\pi}{3}$ & 1 & $\frac{3 \pi}{4}$ & $\frac{3 \pi}{4}$ & $\begin{array}{l}-2.42234 \\
-0.69511 \mathrm{t} \\
-0.69511 \tilde{\mathrm{t}}\end{array}$ & $\begin{array}{c}+2.04473 \\
+0.69572 \mathrm{t} \\
-0.44172 \tilde{\mathrm{t}}\end{array}$ & $\begin{array}{l}-0.50044 \\
-0.00015 \mathrm{t} \\
-0.00014 \tilde{\mathrm{t}}\end{array}$ & $\begin{array}{l}-2.30777 \\
-0.01877 \mathfrak{t} \\
-0.01878 \tilde{\mathfrak{t}}\end{array}$ \\
\hline$\frac{7 \pi}{6}$ & $\frac{5 \pi}{6}$ & $-\frac{\pi}{3}$ & 1 & $\frac{3 \pi}{4}$ & $\frac{3 \pi}{4}$ & $\begin{array}{l}-2.42234 \\
-0.69511 \mathrm{t} \\
-0.69511 \tilde{\mathrm{t}}\end{array}$ & $\begin{array}{l}+1.96819 \\
-0.10269 t \\
-0.10269 \tilde{t}\end{array}$ & $\begin{array}{l}-0.50101 \\
+0.00008 \mathrm{t} \\
+0.00008 \tilde{\mathrm{t}}\end{array}$ & $\begin{array}{l}-2.30374 \\
-0.02007 \mathrm{t} \\
-0.02007 \tilde{\mathrm{t}}\end{array}$ \\
\hline-1 & +1 & 2 & 1 & $\frac{3 \pi}{4}$ & $\frac{3 \pi}{4}$ & $\begin{array}{r}-2.47964 \\
+1.46576 \mathrm{t} \\
+1.46576 \tilde{\mathrm{t}}\end{array}$ & $\begin{array}{l}+3.10442 \\
+0.02602 \mathrm{t} \\
-0.86801 \tilde{\mathrm{t}}\end{array}$ & $\begin{array}{l}-0.49893 \\
-0.00314 \mathrm{t} \\
-0.00336 \tilde{\mathrm{t}}\end{array}$ & $\begin{array}{l}-3.26833 \\
+0.29060 \mathrm{t} \\
+0.29211 \tilde{\mathrm{t}}\end{array}$ \\
\hline$\pi-1$ & $\pi+1$ & 2 & 1 & $\frac{3 \pi}{4}$ & $\frac{3 \pi}{4}$ & $\begin{array}{r}-2.47964 \\
+1.46576 \mathrm{t} \\
+1.46576 \tilde{\mathrm{t}}\end{array}$ & $\begin{array}{l}+2.70455 \\
+0.20706 \mathrm{t} \\
+0.20706 \tilde{\mathrm{t}}\end{array}$ & $\begin{array}{l}-0.49839 \\
-0.00226 \mathrm{t} \\
-0.00226 \tilde{\mathrm{t}}\end{array}$ & $\begin{array}{l}-3.26854 \\
+0.27889 \mathfrak{t} \\
+0.27889 \tilde{\mathrm{t}}\end{array}$ \\
\hline 0 & 0 & 0 & 1 & $-\frac{\pi}{3}$ & $-\frac{\pi}{6}$ & -2.41840 & $\begin{array}{c}+1.81381 \\
-0.07557 \mathfrak{n}_{1} \\
+0.07557 \mathfrak{n}_{2} \\
-0.15115 \mathfrak{n}_{a 1} \\
+0.15115 \mathfrak{n}_{a 2} \\
+0.60460 \mathfrak{t} \\
-0.60460 \tilde{\mathfrak{t}}\end{array}$ & -0.50031 & -2.13942 \\
\hline 0 & 0 & 0 & 1 & $\frac{\pi}{4}$ & $-\frac{\pi}{12}$ & -2.41840 & $\begin{array}{c}+1.81381 \\
+0.15115 \mathfrak{n}_{1} \\
-0.15115 \mathfrak{n}_{2} \\
+0.30230 \mathfrak{n}_{a 1} \\
-0.30230 \mathfrak{n}_{a 2} \\
+0.60460 \mathfrak{t} \\
-0.60460 \tilde{\mathfrak{t}}\end{array}$ & -0.50027 & -2.13970 \\
\hline
\end{tabular}

(b) $k=0 ; \Delta_{a}=\left\{\frac{\pi}{4}, \frac{\pi}{4}, \frac{3 \pi}{4}, \frac{3 \pi}{4}\right\}\left(\Delta=\frac{\pi}{4}\right)$.

\begin{tabular}{lccccccccc}
\hline$\Delta_{m}^{(1)}$ & $\Delta_{m}^{(2)}$ & $\Delta_{m}$ & $n$ & $\Delta_{a 1}$ & $\Delta_{a 2}$ & $f_{1}$ & $f_{2}$ & $f_{3}$ & -2.13933 \\
\hline 0 & 0 & 0 & 1 & $\frac{7 \pi}{8}$ & $\frac{7 \pi}{8}$ & -2.41840 & +1.81381 & -0.50033 & \\
& & & & & & & $-0.60460 \mathrm{t}$ & & \\
& & & & & & & $-0.60460 \tilde{\mathrm{t}}$ & & \\
\hline
\end{tabular}


TABLE V. (Continued)

\begin{tabular}{|c|c|c|c|c|c|c|c|c|c|}
\hline$\Delta_{m}^{(1)}$ & $\Delta_{m}^{(2)}$ & $\Delta_{m}$ & $n$ & $\Delta_{a 1}$ & $\Delta_{a 2}$ & $f_{1}$ & $f_{2}$ & $f_{3}$ & $f_{4}$ \\
\hline$-\pi$ & $-\frac{\pi}{4}$ & $\frac{3 \pi}{4}$ & 2 & $-\frac{\pi}{4}$ & 0 & $\begin{array}{l}-3.42919 \\
+0.55815 \mathrm{t} \\
+0.55815 \tilde{\mathrm{t}}\end{array}$ & $\begin{array}{c}+1.47608 \\
-0.34209 \mathfrak{n}_{1} \\
+0.34209 \mathfrak{n}_{2} \\
-0.56442 \mathfrak{n}_{a 1} \\
+0.56442 \mathfrak{n}_{a 2} \\
+0.61625 \mathfrak{t} \\
-0.66691 \tilde{\mathfrak{t}}\end{array}$ & $\begin{array}{c}-0.49693 \\
-0.00524 \mathfrak{n}_{1} \\
+0.00524 \mathfrak{n}_{2} \\
-0.00103 \mathfrak{n}_{a 1} \\
+0.00103 \mathfrak{n}_{a 2} \\
-0.00517 \mathfrak{t} \\
-0.00581 \tilde{\mathfrak{t}}\end{array}$ & $\begin{array}{c}-2.08016 \\
+0.03162 \mathfrak{n}_{1} \\
-0.03162 \mathfrak{n}_{2} \\
+0.00615 \mathfrak{n}_{a 1} \\
-0.00615 \mathfrak{n}_{a 2} \\
-0.08824 \mathfrak{t} \\
-0.08445 \tilde{\mathfrak{t}}\end{array}$ \\
\hline$\frac{3 \pi}{2}$ & 0 & $-\frac{3 \pi}{2}$ & 3 & $\frac{3 \pi}{2}$ & $\frac{3 \pi}{4}$ & $\begin{array}{l}-4.22590 \\
-0.62974 t \\
-0.62974 \tilde{\mathrm{t}}\end{array}$ & $\begin{array}{c}+1.26660 \\
+1.34234 \mathfrak{n}_{1} \\
-1.34234 \mathfrak{n}_{2} \\
+2.76857 \mathfrak{n}_{a 1} \\
-2.76857 \mathfrak{n}_{a 2} \\
+0.02775 \mathfrak{t} \\
-0.12140 \tilde{\mathfrak{t}}\end{array}$ & $\begin{array}{c}-0.49950 \\
+0.00018 \mathfrak{n}_{1} \\
-0.00018 \mathfrak{n}_{2} \\
+0.00056 \mathfrak{n}_{a 1} \\
-0.00056 \mathfrak{n}_{a 2} \\
+0.00088 \mathfrak{t} \\
+0.00085 \tilde{\mathfrak{t}}\end{array}$ & $\begin{array}{c}-1.95975 \\
-0.00141 \mathfrak{n}_{1} \\
+0.00141 \mathfrak{n}_{2} \\
-0.00445 \mathfrak{n}_{a 1} \\
+0.00445 \mathfrak{n}_{a 2} \\
+0.25498 \mathfrak{t} \\
+0.25520 \tilde{\mathrm{t}}\end{array}$ \\
\hline
\end{tabular}

\begin{tabular}{|c|c|c|c|c|c|c|c|c|c|}
\hline \multicolumn{10}{|c|}{ (c) $k=0 ; \Delta_{a}=\{2,2, \pi-2, \pi-2\}(\Delta=2)$} \\
\hline$\underline{\Delta_{m}^{(1)}}$ & $\Delta_{m}^{(2)}$ & $\Delta_{m}$ & $n$ & $\Delta_{a 1}$ & $\Delta_{a 2}$ & $f_{1}$ & $f_{2}$ & $f_{3}$ & $f_{4}$ \\
\hline 0 & 0 & 0 & 1 & $\pi-1$ & $\pi-1$ & -2.41840 & $\begin{array}{l}+1.81381 \\
+0.60460 \mathrm{t} \\
-060460 \mathrm{t}\end{array}$ & -0.50033 & -2.13933 \\
\hline$\frac{\pi}{3}$ & $\frac{5 \pi}{3}$ & $\frac{4 \pi}{3}$ & 2 & $-\frac{1}{2}$ & $-\frac{3}{2}$ & $\begin{array}{l}-3.52661 \\
+1.10095 \mathrm{t} \\
+1.10095 \tilde{\mathrm{t}}\end{array}$ & $\begin{array}{c}+1.58880 \\
+0.24574 \mathfrak{n}_{1} \\
-0.24574 \mathfrak{n}_{2} \\
+0.49147 \mathfrak{n}_{a 1} \\
-0.49147 \mathfrak{n}_{a 2} \\
+0.40646 \mathrm{t} \\
+0.40646 \tilde{\mathfrak{t}}\end{array}$ & $\begin{array}{c}-0.49533 \\
+0.00005 \mathfrak{n}_{1} \\
-0.00005 \mathfrak{n}_{2} \\
+0.00009 \mathfrak{n}_{a 1} \\
-0.00009 \mathfrak{n}_{a 2} \\
-0.00387 \mathfrak{t} \\
-0.00387 \tilde{\mathfrak{t}}\end{array}$ & $\begin{array}{c}-0.49533 \\
+0.00005 \mathfrak{n}_{1} \\
-0.00005 \mathfrak{n}_{2} \\
+0.00009 \mathfrak{n}_{a 1} \\
-0.00009 \mathfrak{n}_{a 2} \\
-0.00387 \mathfrak{t} \\
-0.00387 \tilde{\mathfrak{t}}\end{array}$ \\
\hline 2 & -1 & -3 & 3 & $4 \pi-12$ & $6-2 \pi$ & $\begin{array}{l}-4.19444 \\
-0.38206 \mathfrak{t} \\
-0.38206 \tilde{\mathfrak{t}}\end{array}$ & $\begin{array}{c}+0.94252 \\
+0.54645 \mathfrak{n}_{1} \\
-0.54645 \mathfrak{n}_{2} \\
+1.16441 \mathfrak{n}_{a 1} \\
-1.16441 \mathfrak{n}_{a 2} \\
+0.06507 \mathfrak{t} \\
-0.49034 \tilde{\mathfrak{t}}\end{array}$ & $\begin{array}{c}-0.50128 \\
+0.00333 \mathfrak{n}_{1} \\
-0.00333 \mathfrak{n}_{2} \\
+0.01182 \mathfrak{n}_{a 1} \\
-0.01182 \mathfrak{n}_{a 2} \\
+0.00218 \mathfrak{t} \\
+0.00163 \tilde{\mathfrak{t}}\end{array}$ & $\begin{array}{c}-2.10032 \\
-0.01903 \mathfrak{n}_{1} \\
+0.01903 \mathfrak{n}_{2} \\
-0.07214 \mathfrak{n}_{a 1} \\
+0.07214 \mathfrak{n}_{a 2} \\
+0.15335 \mathfrak{t} \\
+0.15687 \tilde{\mathfrak{t}}\end{array}$ \\
\hline
\end{tabular}

$$
\begin{aligned}
f(N)= & f_{1} N^{3 / 2}+f_{2} N^{1 / 2}+f_{3} \log N+f_{4} \\
& +\sum_{p=1}^{p_{c}} f_{p+4} N^{(1-2 p) / 2} .
\end{aligned}
$$

The results of the numerical fit for $\operatorname{Re} \log Z$ with $N$ are presented in Table V. The analytical leading term computed by the index theorem in [26] and the numerical leading term $f_{1} N^{3 / 2}$ match to number of significant digits present in the table. And the leading term is indeed independent of $\left\{\Delta_{m}^{(1)}, \Delta_{m}^{(2)}\right\}$ keeping the same $\Delta_{m}$, and $\left\{\Delta_{a 1}, \Delta_{a 2}, \tilde{\Delta}_{a 1}\right.$, $\left.\tilde{\Delta}_{a 2}\right\}$. The numerical results indicate that the coefficient $f_{3}$ of the $\log N$ term is precisely $-1 / 2$.

\section{ONE-LOOP ENTROPY IN ELEVEN DIMENSIONAL SUPERGRAVITY}

Inspired by the seminal work of ABJM [34] who established the now prototypical dual pair of $\left(\mathrm{AdS}_{4} \times S^{7} /\right.$ $\left.\mathbb{Z}_{k}\right) / \mathrm{CFT}_{3}$ where $\mathrm{CFT}_{3}$ stands for the particular ChernSimons matter theory discussed in Sec. II, a plethora of similar examples was constructed. A natural way to establish new dual pairs is to consider, on the gravity side, appropriate manifolds that could replace the seven-sphere, $S^{7}$. The starting point are Freund-Rubin type solutions of the form $\mathrm{AdS}_{4} \times M^{7}$ for a certain list of seven-dimensional Sasaki-Einstein spaces, $M^{7}$ [49]. A fairly complete description of solutions of seven dimensional manifolds, providing 
Freund-Ruben solutions to $11 \mathrm{~d}$ supergravity, was cataloged by Duff, Nilsson and Pope in [50] (see a previous discussion in [51]). The list includes further specification about those which are supersymmetric and states what fraction of the supersymmetry is preserved. An exhaustive list of Sasaki-Einstein seven-dimensional manifolds is presented in [52]. Some prominent cases in the list include $M^{7}=$ $\left\{S^{7}, Q^{1,1,1}, M^{1,1,1}, V^{5,2}, N^{0,1,0}\right\}$ and their quotients by $\mathbb{Z}_{k}$. The typical structure of those manifolds is that of toric Sasaki-Einstein manifolds and can be written as a $U(1)$ bundled over a Kaehler-Einstein base. For example, $M^{1,1,1}$ is geometrically a $U(1)$ bundle over $\mathbb{C P}^{2} \times S^{2}$, the dual quiver Chern-Simons matter theory was discussed in [53,54]; $Q^{1,1,1}$ is geometrically a $U(1)$ bundle over $S^{2} \times S^{2} \times S^{2}$, the dual theory is an $\mathcal{N}=2$ supersymmetric Chern-Simons matter quiver gauge theory $[43,44,47,47,48,55]$. The one nontoric case in the list $\mathrm{AdS}_{4} \times V^{5,2}$ was addressed in $[42,44]$. For all these dual pairs the free energy of the field theory on $S^{3}$ was shown to agree with the regularized on-shell action on the gravity side largely using techniques presented in [56] (see also [57] for recent applications). More recently, the topologically twisted index of a number of these field theories has been computed $[25,26,58,59]$.

Our goal in this section is to compute the logarithmic correction to the entropy of the magnetically charged black holes dual to the field theory computations presented in the previous sections and establish that it coincides with the result of the field theory side. To compute such logarithmic corrections one requires only low energy data, that is, only the spectrum of massless fields which in this case would be eleven-dimensional supergravity with background asymptoting to the Freund-Rubin spaces mentioned above plus magnetic flux components. These IR corrections provide a litmus test for the would-be UV complete description of gravity which in our case are simply the Chern-Simons matter field theories discussed in the previous sections. Such powerful IR window into UV physics was studied by Ashoke Sen and collaborators in the case of asymptotically flat string theory black holes $[60,61]$; in this case string theory provides the UV complete result and the IR results are, again, furnished by supergravity theories. In the context of the AdS/CFT correspondence, there have been some developments in matching the gravity computation to the coefficient of $\log N$ term on the field theory side [13,20-22,41,62-65]. For the cases of AdS/CFT pairs arising from M5 branes wrapping hyperbolic three-manifolds, the field theory results were obtained analytically and shown to match the gravity result in $[13,19]$. We are, nevertheless, quite confident in the numerical results presented here and in previous works $[20,41,63]$.

In this section we compute the one-loop logarithmic correction from the gravity side and confront them with the field-theoretic (UV) results. Let us start by recalling a number of important facts regarding the one-loop effective actions of supergravity backgrounds. Our setup is $11 \mathrm{~d}$ supergravity where we assume there is an embedding of the solutions describing magnetically charged asymptotically $\mathrm{AdS}_{4} \times M^{7}$ black holes.

We make the assumption that the whole contribution to the one-loop effective action comes from the asymptotic $\mathrm{AdS}_{4}$ region as was the case in [24] for the $\mathrm{AdS}_{4}$ solution and in [22], for the magnetically charged asymptotically $\mathrm{AdS}_{4}$ black hole case and for black holes described by M5 branes wrapping hyperbolic 3-manifolds in $[13,19]$.

On very general grounds of diffeomorphism invariance, it can be argued that in odd-dimensional spacetimes, the top Seeley-De Witt coefficient $a_{d / 2}$ vanishes [66]. Therefore, the only contribution to the heat kernel comes from the zero modes. Applied to our case, the one-loop contribution due to $11 \mathrm{~d}$ supergravity comes from the analysis of zero modes. As in previous cases [13,19,22,24], the gravity computation performed in 11d sugra is essentially reduced to the contribution of a two-form zero mode in the asymptotically $\mathrm{AdS}_{4} \times M^{7}$ region.

More explicitly, given that there is a two-form zero mode in $\mathrm{AdS}_{4}$ we need to make sure that there are possible zero modes in $M^{7}$ that could contribute. In the spectrum of quantum eleven-dimensional supergravity we can have contribution coming from one-form zero modes (ghost), two-form zero modes (ghost) and three-form zero modes $\left(C_{3}\right)$. Other than the two-form zero modes discussed already in [22], there is another potential source of zero modes which could arise if $M^{7}$ admits a harmonic oneform. This one-form zero mode could contribute to the ghost one-form or it could contribute to the harmonic threeform on $\mathrm{AdS}_{4} \times M^{7}$ by taking the wedge product of a harmonic two-form on $\mathrm{AdS}_{4}$ times a harmonic one-form on $M^{7}$. It is worth pointing that, given the magnetic charges, the space is not really a direct product but there is a fibering of $M^{7}$ over $\mathrm{AdS}_{4}$. This fibering was studied in detail in [20] and shown to not affect the counting of two-form zero modes relevant in this section.

We will not reproduce all the details of the computation here, the interested reader is referred to [13,19,22] for details. We briefly sketch the derivation of the one-loop effective action. Given that the only zero mode in $\mathrm{AdS}_{4}$ is a 2 -form and assuming that the solution is asymptotically of the form $\mathrm{AdS}_{4} \times M^{7}$ we need to decompose the kinetic operator along these two subspaces. For the 2-form zero mode of $\mathrm{AdS}_{4}$ to survive we need to have the corresponding part of the kinetic Laplace-like operator also vanishing.

When integrating over zero modes there is a factor of $L^{ \pm \beta_{A}}$ for each zero mode in the path integral. The total contribution to the partition function from the zero modes is

$$
L^{ \pm \beta_{A} n_{A}^{0}}
$$

where $n_{A}^{0}$ is the number of zero modes of the kinetic operator $A$ and the sign depends on whether the operator is fermionic or bosonic. Typically, zero modes are associated with certain asymptotic symmetries. For example, with 
gauge transformations that do not vanish at infinity. The key idea in determining $\beta_{A}$ is to find the right variables of integrations and to count the powers of $L$ that such integration measure contributes. The scaling exponent for $p$-forms is easily computed [24], yielding $\beta_{p}=(d-$ $2 p) / 2$ in terms of the total dimension $d$ of spacetime. For the case at hand of a 2 -form in eleven dimensions, we have $\beta_{2}=(11-4) / 2=7 / 2$.

Having determined $\beta_{2}$, the computation of the one-loop effective action reduces to counting the number of 2 -form zero modes, $n_{2}^{0}$. A simple way to determine the number of 2-form zero modes is by computing the Euler characteristic of the black hole. In [13,22] it was argued that $n_{2}^{0}=$ $2(1-g)$ for a black hole of horizon given by a genus $g$ Riemann surface. Note that this number is computed using the non-extremal branch of the solution and that it is independent of the charges of the black holes. Therefore, be it for the magnetically charged or the electrically charged black holes we obtain the same result.

The full contribution to the logarithmic terms of the oneloop effective action is thus given only by the 2 -form zero modes and we have:

$$
\log Z_{1-\operatorname{loop}}=\left(2-\beta_{2}\right) n_{2}^{0} \log L=(2-7 / 2) 2(1-g) \log L=\frac{1}{2}(g-1) \log N \text {, }
$$

where according to the AdS/CFT dictionary we have used that for M2 branes backgrounds we have $L^{6} \sim N$. When restricting to spherically symmetric horizons $(g=0)$ we find perfect agreement with the numerical field theory results in previous sections. The topologically twisted index result in the previous sections assume $g=0$ but it is easily generalized to arbitrary $g$ and the agreement with Eq. (6.2) remains robust.

There is a generalization of the above result, obtained in [13] and [19], for the case where $M^{7}$ has nonvanishing first Betti number, $b_{1}$. The generalization takes the form

$$
\log Z_{1-\operatorname{loop}}=\frac{1}{2}(g-1)\left(1-b_{1}\right) \log N,
$$

and was shown to match the field theory result for certain $M^{7}$ constructed as 4-sphere fibration over a hyperbolic 3-manifold [19]. The extra contribution proportional to $b_{1}$ arises from the supergravity 3 -form potential as one can construct a zero mode by combining the 2 -form zero mode in $\mathrm{AdS}_{4}$ and a 1-form zero mode in the hyperbolic 3-manifold.

For complete agreement between gravity and field theory, we need to show the vanishing of the first Betti number for the $M^{7}$ we considered in this manuscript. This can be shown as follows. Every seven-dimensional, compact Einstein manifold of positive curvature has vanishing first Betti number (see, for example, [50]). This can be seen from the Hodge-de Rham operator acting on one-forms:

$$
\Delta_{1} Y_{m}=\square Y_{m}+R_{m}{ }^{n} Y_{n}
$$

Recall that the Hodge-de Rham operator is defined as

$$
\Delta=d \delta+\delta d
$$

where $d$ is the exterior differentiation mapping $p$-forms to $(p+1)$-forms and $\delta=(-1)^{p} * d *$ is its adjoint where $*$ is the Hodge dual operation. Let us assume that the Einstein manifold $M^{7}$ has natural normalization, $R_{m n}=6 m^{2} g_{m n}$. Considering the eigenvalues

$$
\Delta_{1} V_{m}=\lambda V_{m},
$$

it follows immediately that $\Delta_{1} \geq 6 \mathrm{~m}^{2}$. For one-forms that are coclosed $\nabla^{m} V_{m}=0$ one can prove an even stronger bound. Therefore, for the class of Sasaki-Einstein sevenmanifolds relevant for our analysis we have vanishing first Betti number and, subsequently perfect agreement of the logarithmic term in Eq. (6.2) with the field theory results in the previous sections.

Let us finish this section with one important remark. The analysis performed in this section relied only on the asymptotic form of the black hole background. The explicit construction of such black hole backgrounds is, however, a highly nontrivial problem. In the case of $S^{7}$ many results exists in the literature for very general black holes. The case of $Q^{1,1,1}$ has been widely discussed with relatively modest results about the near-horizon region presented in [11,67-69].

\section{CONCLUSIONS}

In this manuscript we have numerically studied the topologically twisted index of various Chern-Simons matter quiver gauge theories on the product of a genus $g$ Riemann surface and the circle, $\Sigma_{g} \times S^{1}$ and determined that, in all cases, there is a logarithmic contribution of the from $\frac{g-1}{2} \log N$. We are able to explicitly track the contributions to the logarithmic terms coming from different elements of the index including the precise cancellation of $N \log N$ contributions between the vector multiplet and the Jacobian contribution to the topologically twisted index. We have also provided the dual computation of one-loop quantum supergravity which perfectly matches the field theory result. This gravity computation is quite universal 
and requires a mild cohomological property (vanishing of first Betti number, $b_{1}=0$ ) on the dual seven-dimensional manifold $M^{7}$ which is satisfied for most of the examples discussed in this manuscript.

The universality of our result for the topologically twisted index of $3 \mathrm{~d}$ theories was inspired by the universality of the free energy on $S^{3}$ discussed in $[23,24]$. This universality also interestingly resonates with a recent analogous study in four dimensions which analytically showed that there is a universal logarithmic contribution to the superconformal index of a large class of $4 \mathrm{~d} \mathcal{N}=1$ supersymmetric field theories [70]. Perhaps similar universal results exist in other dimensions.

We expect that our supergravity analysis extends to rotating electrically charged asymptotically $\mathrm{AdS}_{4} \times M^{7}$ black holes beause the result is independent of the black hole charges and depends only on the dictionary entry relating Newton's contanst, $G_{N}$, to the rank of the gauge group, $N$, and the horizon topology. For the case of theories obtained from M5 branes wrapping three-dimensional hyperbolic manifolds, the logarithmic counting for magnetically charged black holes was presented in [13]; the case of rotating, electrically charged black holes was analyzed in [19]. In both cases the logarithmic term in the field theory side was known analytically and the supergravity analysis was essentially the same and the result was independent of the black hole charges. Indeed, it is clear that the logarithmic computation as presented here and in previous works is independent of the charges. Thus, we claim that our analysis here is also valid for all asymptotically $\mathrm{AdS}_{4} \times M^{7}$ black holes whether magnetically charged or rotating, electrically charged ones. It would be interesting to directly verify this claim by analyzing the logarithmic term in the superconformal index of these theories.

It would be interesting to understand our results from a more analytic point of view. A natural starting point could be by pursuing the relation between the Bethe potential $\mathcal{V}$ and the expectation value of the free energy on $S^{3}$ as pointed out in [25] but beyond the leading order. There are other more formal arguments establishing a relation between the topologically twisted index in $S^{2} \times S^{1}$ and the free energy on $S^{3}$ pointed out in [27]. Namely, the leading in $N$ relations between the free energy on $S^{3}$ and the topologically twisted index has been well documented $[25,26]$ by explicit computations. Quite remarkably, certain universality of the logarithmic terms in the free energy on $S^{3}$ of a large class of Chern-Simons matter theories was established in [23], that is, a universal contribution of the form $-\frac{1}{4} \log N$; the dual supergravity side was elucidated in [24] and found to be in perfect agreement. Our result in this manuscript - the universality of $-\frac{1}{2} \log N$, is mostly numerical. It would be interesting to develop a matrix model intuition into some of the crucial subleading in $N$ relations between the free energy on $S^{3}$ and the topologically twisted index on $\Sigma_{g} \times S^{1}$ for this large class of field theories. It will also be quite natural to include aspects of the superconformal index as presented in $[16,17,71]$ in this universality analysis. We hope to report on these efforts.

We have studied various theories that have M-theory duals. It would be interesting to extend our result to field theories admitting massive IIA duals where the growth of the microstates goes as $N^{5 / 3}$. On the field theory side one focuses on the topologically twisted index of $S U(N)$ Chern-Simons matter theory at level $k$ whose leading term, of order $N^{5 / 3}$, coincides with the entropy of magnetically charged, asymptotically $\mathrm{AdS}_{4} \times S^{6}$ black holes in massive type IIA theory $[4,5]$. The black holes in question were presented in [72] as a payoff of the arduous work of obtaining $\mathrm{AdS}_{4}$ gauged supergravity from the reduction of massive type IIA theory [73-76]. The log term in this Chern-Simons matter theory was computed in [63] using a combination of analytical and numerical techniques, it would be interesting to extend those results to a larger class of theories where a similar universality might be established. The gravity computation of the logarithmic contribution, it merits to say, is quite more complicated due to the dual theory living in an even-dimensional space leading to a more general type of contributions to the logarithmic term.

Another potentially fruitful avenue would be to explore the 't Hooft limit where $N \rightarrow \infty$ with $\lambda=N / k$ kept fixed. To the best of our knowledge, there are no results about this limit for the topologically twisted index other than the analysis of [41]. Even for the free energy on $S^{3}$ we are not aware of systematic numerical explorations beyond the large $N$ leading term. It is worth noticing that in this limit one expects a re-arrangement of the degrees of freedoms as guided by the scaling of the free energy. On the gravity side, subleading corrections are also quite different as the one-loop quantum supergravity computations now depend on more dynamical aspects of the background given that the dual gravity leaves in ten-dimensional type IIA supergravity.

We have not addressed in any detail the subleading $N^{1 / 2}$ behavior which corresponds to higher curvature corrections on the gravity side. For the case of the ABJM theory, the $N^{1 / 2}$ was determine in a combination of numerical and analytical approaches in [20]. A number of interesting bottom-up observations regarding the structure of higher curvature corrections in similar classes of theories were made recently in [77] and it would be interesting to pursue this entry in the AdS/CFT dictionary more precisely in this context. We hope to report on some explorations along these lines.

Finally, there is a glaring open challenge to the supergravity community-the problem of missing black holes. There are some approaches that allow one to determine the entropy of the supergravity dual black holes to certain 
quiver Chern-Simons matter theory (see, for example, [78-80]). Some progress has also been reported in $[11,81,82]$. Our discussion in Sec. VI assumes the existence of such black holes and demonstrate that the logarithmic corrections to the entropy precisely matches the field theory results using general aspects of the would-be black hole solution. All these impressive tests are performed in the backdrop where the explicit construction of the black holes is lacking. It remains a very interesting question to explicitly find those black holes and compute their Bekenstein-Hawking entropy and demonstrate that it agrees with the microscopic prediction of the topologically twisted index.

\section{ACKNOWLEDGMENTS}

We are thankful to Francesco Benini, Chandramouli Chowdhury, Marina David, Dongmin Gang, Jewel Ghosh, Alfredo González Lezcano, Junho Hong, Sayed M. Hosseini, Albrecht Klemm, James T. Liu, Jun Nian, Vimal Rathee, Ashoke Sen, Wenli Zhao and Shan Zhou. This work was supported in part by the U.S. Department of Energy under Grant No. DE-SC0007859.
[1] F. Benini, K. Hristov, and A. Zaffaroni, Black hole microstates in $\mathrm{AdS}_{4}$ from supersymmetric localization, J. High Energy Phys. 05 (2016) 054.

[2] F. Benini, K. Hristov, and A. Zaffaroni, Exact microstate counting for dyonic black holes in $\mathrm{AdS}_{4}$, Phys. Lett. B 771, 462 (2017).

[3] A. Cabo-Bizet, V. I. Giraldo-Rivera, and L. A. Pando Zayas, Microstate counting of $\mathrm{AdS}_{4}$ hyperbolic black hole entropy via the topologically twisted index, J. High Energy Phys. 08 (2017) 023.

[4] F. Benini, H. Khachatryan, and P. Milan, Black hole entropy in massive type IIA, Classical Quantum Gravity 35, 035004 (2018).

[5] S. M. Hosseini, K. Hristov, and A. Passias, Holographic microstate counting for $\mathrm{AdS}_{4}$ black holes in massive IIA supergravity, J. High Energy Phys. 10 (2017) 190.

[6] S. M. Hosseini, I. Yaakov, and A. Zaffaroni, Topologically twisted indices in five dimensions and holography, J. High Energy Phys. 11 (2018) 119.

[7] P. M. Crichigno, D. Jain, and B. Willett, 5d partition functions with a twist, J. High Energy Phys. 11 (2018) 058.

[8] M. Suh, Supersymmetric $\mathrm{AdS}_{6}$ black holes from $\mathrm{F}(4)$ gauged supergravity, J. High Energy Phys. 01 (2019) 035.

[9] S. M. Hosseini, K. Hristov, A. Passias, and A. Zaffaroni, 6D attractors and black hole microstates, J. High Energy Phys. 12 (2018) 001.

[10] M. Suh, Supersymmetric $\mathrm{AdS}_{6}$ black holes from matter coupled $F(4)$ gauged supergravity, J. High Energy Phys. 02 (2019) 108.

[11] F. Azzurli, N. Bobev, P. M. Crichigno, V. S. Min, and A. Zaffaroni, A universal counting of black hole microstates in $\mathrm{AdS}_{4}$, J. High Energy Phys. 02 (2018) 054.

[12] D. Gang and N. Kim, Large $N$ twisted partition functions in 3d-3d correspondence and Holography, Phys. Rev. D 99, 021901 (2019).

[13] D. Gang, N. Kim, and L. A. Pando Zayas, Precision microstate counting for the entropy of wrapped M5-branes, J. High Energy Phys. 03 (2020) 164.

[14] S. M. Hosseini, Black hole microstates and supersymmetric localization, Ph.D. Thesis, Milan Bicocca University, 2018.
[15] A. Zaffaroni, Lectures on AdS black holes, holography and localization, Living Rev. Relativity 23, 2 (2020).

[16] S. Choi, C. Hwang, and S. Kim, Quantum vortices, M2branes and black holes, arXiv:1908.02470.

[17] J. Nian and L. A. Pando Zayas, Microscopic entropy of rotating electrically charged $\mathrm{AdS}_{4}$ black holes from field theory localization, J. High Energy Phys. 03 (2020) 081.

[18] N. Bobev and P. M. Crichigno, Universal spinning black holes and theories of class $\mathcal{R}$, J. High Energy Phys. 12 (2019) 054.

[19] F. Benini, D. Gang, and L. A. Pando Zayas, Rotating black hole entropy from M5 branes, J. High Energy Phys. 03 (2020) 057.

[20] J. T. Liu, L. A. Pando Zayas, V. Rathee, and W. Zhao, Toward microstate counting beyond large $\mathrm{N}$ in localization and the dual one-loop quantum supergravity, J. High Energy Phys. 01 (2018) 026.

[21] I. Jeon and S. Lal, Logarithmic corrections to entropy of magnetically charged $\mathrm{AdS}_{4}$ black holes, Phys. Lett. B 774, 41 (2017).

[22] J. T. Liu, L. A. Pando Zayas, V. Rathee, and W. Zhao, OneLoop Test of Quantum Black Holes in Antide Sitter Space, Phys. Rev. Lett. 120, 221602 (2018).

[23] M. Marino and P. Putrov, ABJM theory as a Fermi gas, J. Stat. Mech. (2012) P03001.

[24] S. Bhattacharyya, A. Grassi, M. Marino, and A. Sen, A oneloop test of quantum supergravity, Classical Quantum Gravity 31, 015012 (2014).

[25] S. M. Hosseini and A. Zaffaroni, Large $N$ matrix models for $3 \mathrm{~d} \mathcal{N}=2$ theories: Twisted index, free energy and black holes, J. High Energy Phys. 08 (2016) 064.

[26] S. M. Hosseini and N. Mekareeya, Large $N$ topologically twisted index: Necklace quivers, dualities, and SasakiEinstein spaces, J. High Energy Phys. 08 (2016) 089.

[27] C. Closset, H. Kim, and B. Willett, Supersymmetric partition functions and the three-dimensional A-twist, J. High Energy Phys. 03 (2017) 074.

[28] C. Toldo and B. Willett, Partition functions on $3 \mathrm{~d}$ circle bundles and their gravity duals, J. High Energy Phys. 05 (2018) 116. 
[29] C. Closset, H. Kim, and B. Willett, Seifert fibering operators in $3 \mathrm{~d} \mathcal{N}=2$ theories, J. High Energy Phys. 11 (2018) 004.

[30] F. Benini and A. Zaffaroni, A topologically twisted index for three-dimensional supersymmetric theories, J. High Energy Phys. 07 (2015) 127.

[31] M. Honda and Y. Yoshida, Supersymmetric index on $T^{2} \times$ $S^{2}$ and elliptic genus, arXiv:1504.04355.

[32] C. Closset, S. Cremonesi, and D. S. Park, The equivariant A-twist and gauged linear sigma models on the two-sphere, J. High Energy Phys. 06 (2015) 076.

[33] C. Closset and H. Kim, Comments on twisted indices in 3d supersymmetric gauge theories, J. High Energy Phys. 08 (2016) 059.

[34] O. Aharony, O. Bergman, D. L. Jafferis, and J. Maldacena, $\mathcal{N}=6$ superconformal Chern-Simons-matter theories, M2branes and their gravity duals, J. High Energy Phys. 10 (2008) 091.

[35] D. Fabbri, P. Fre', L. Gualtieri, C. Reina, A. Tomasiello, A. Zaffaroni, and A. Zampa, 3-D superconformal theories from Sasakian seven manifolds: New nontrivial evidences for AdS(4)/CFT(3), Nucl. Phys. B577, 547 (2000).

[36] M. Billo, D. Fabbri, P. Fre, P. Merlatti, and A. Zaffaroni, Rings of short $N=3$ superfields in three-dimensions and $\mathrm{M}$ theory on $A d s_{4} \times N^{0,1,0}$, Classical Quantum Gravity 18, 1269 (2001).

[37] H.-U. Yee, AdS/CFT with Tri-Sasakian manifolds, Nucl. Phys. B774, 232 (2007).

[38] D. Gaiotto and D. L. Jafferis, Notes on adding D6 branes wrapping $\mathbb{R P}^{3}$ in $A d s_{4} \times \mathbb{C P}^{3}$, J. High Energy Phys. 11 (2012) 015.

[39] Y. Imamura, D. Yokoyama, and S. Yokoyama, Superconformal index for large $\mathrm{N}$ quiver Chern-Simons theories, J. High Energy Phys. 08 (2011) 011.

[40] S. Cheon, D. Gang, S. Kim, and J. Park, Refined test of AdS4/CFT3 correspondence for $N=2,3$ theories, J. High Energy Phys. 05 (2011) 027.

[41] L. A. Pando Zayas and Y. Xin, Topologically twisted index in the 't Hooft limit and the dual $\mathrm{AdS}_{4}$ black hole entropy, Phys. Rev. D 100, 126019 (2019).

[42] D. Martelli and J. Sparks, AdS(4)/CFT(3) duals from M2branes at hypersurface singularities and their deformations, J. High Energy Phys. 12 (2009) 017.

[43] D. L. Jafferis, Quantum corrections to $\mathcal{N}=2$ ChernSimons theories with flavor and their $\mathrm{AdS}_{4}$ duals, J. High Energy Phys. 08 (2013) 046.

[44] S. Cheon, H. Kim, and N. Kim, Calculating the partition function of $N=2$ gauge theories on $S^{3}$ and AdS/CFT correspondence, J. High Energy Phys. 05 (2011) 134.

[45] D. Martelli and J. Sparks, The large N limit of quiver matrix models and Sasaki-Einstein manifolds, Phys. Rev. D 84, 046008 (2011).

[46] D. L. Jafferis, I. R. Klebanov, S. S. Pufu, and B. R. Safdi, Towards the F-theorem: $N=2$ field theories on the threesphere, J. High Energy Phys. 06 (2011) 102.

[47] F. Benini, C. Closset, and S. Cremonesi, Chiral flavors and M2-branes at toric CY4 singularities, J. High Energy Phys. 02 (2010) 036.

[48] S. Cremonesi, Type IIB construction of flavoured ABJ(M) and fractional M2 branes, J. High Energy Phys. 01 (2011) 076 .
[49] P. G. O. Freund and M. A. Rubin, Dynamics of dimensional reduction, Phys. Lett. B 97, 233 (1980).

[50] M. J. Duff, B. E. W. Nilsson, and C. N. Pope, Kaluza-Klein supergravity, Phys. Rep. 130, 1 (1986).

[51] L. Castellani, L. Romans, and N. Warner, A classification of compactifying solutions for $d=11$ supergravity, Nucl. Phys. B241, 429 (1984).

[52] T. Friedrich and I. Kath, Seven-dimensional compact Riemannian manifolds with killing spinors, Commun. Math. Phys. 133, 543 (1990).

[53] D. Martelli and J. Sparks, Moduli spaces of Chern-Simons quiver gauge theories and AdS(4)/CFT(3), Phys. Rev. D 78, 126005 (2008).

[54] A. Hanany and A. Zaffaroni, Tilings, Chern-Simons theories and M2 branes, J. High Energy Phys. 10 (2008) 111.

[55] S. Franco, I. R. Klebanov, and D. Rodriguez-Gomez, M2branes on orbifolds of the cone over $Q^{1,1,1}$, J. High Energy Phys. 08 (2009) 033.

[56] C. P. Herzog, I. R. Klebanov, S. S. Pufu, and T. Tesileanu, Multi-matrix models and tri-Sasaki Einstein spaces, Phys. Rev. D 83, 046001 (2011).

[57] A. Amariti, M. Fazzi, N. Mekareeya, and A. Nedelin, New $3 \mathrm{~d} \mathcal{N}=2$ SCFT's with $N^{3 / 2}$ scaling, J. High Energy Phys. 12 (2019) 111.

[58] D. Jain and A. Ray, $3 \mathrm{~d} \mathcal{N}=2 \widehat{A D E}$ Chern-Simons quivers, Phys. Rev. D 100, 046007 (2019).

[59] D. Jain, Twisted indices of more 3d quivers, arXiv: 1908.03035.

[60] A. Sen, Logarithmic corrections to $\mathcal{N}=2$ black hole entropy: An infrared window into the microstates, Gen. Relativ. Gravit. 44, 1207 (2012).

[61] A. Sen, Logarithmic corrections to rotating extremal black hole entropy in four and five dimensions, Gen. Relativ. Gravit. 44, 1947 (2012).

[62] K. Hristov, I. Lodato, and V. Reys, On the quantum entropy function in 4d gauged supergravity, J. High Energy Phys. 07 (2018) 072.

[63] J. T. Liu, L. A. Pando Zayas, and S. Zhou, Subleading microstate counting in the dual to massive type IIA, arXiv:1808.10445.

[64] K. Hristov, I. Lodato, and V. Reys, One-loop determinants for black holes in 4d gauged supergravity, J. High Energy Phys. 11 (2019) 105.

[65] J. Nian and X. Zhang, Entanglement entropy of ABJM theory and entropy of topological black hole, J. High Energy Phys. 07 (2017) 096.

[66] D. V. Vassilevich, Heat kernel expansion: User's manual, Phys. Rep. 388, 279 (2003).

[67] A. Donos, J. P. Gauntlett, and N. Kim, AdS solutions through transgression, J. High Energy Phys. 09 (2008) 021.

[68] N. Halmagyi, M. Petrini, and A. Zaffaroni, BPS black holes in $\mathrm{AdS}_{4}$ from M-theory, J. High Energy Phys. 08 (2013) 124.

[69] J. Hong, N. T. Macpherson, and L. A. Pando Zayas, Aspects of $\mathrm{AdS}_{2}$ classification in M-theory: Solutions with mesonic and baryonic charges, J. High Energy Phys. 11 (2019) 127.

[70] A. González Lezcano, J. Hong, J. T. Liu, and L. A. Pando Zayas, Sub-leading structures in superconformal indices: Subdominant saddles and logarithmic contributions, arXiv: 2007.12604. 
[71] S. Choi and C. Hwang, Universal 3d cardy block and black hole entropy, J. High Energy Phys. 03 (2020) 068.

[72] A. Guarino and J. Tarrio, BPS black holes from massive IIA on $S^{6}$, J. High Energy Phys. 09 (2017) 141.

[73] A. Guarino, D. L. Jafferis, and O. Varela, String Theory Origin of Dyonic $\mathcal{N}=8$ Supergravity and Its ChernSimons Duals, Phys. Rev. Lett. 115, 091601 (2015).

[74] A. Guarino and O. Varela, Dyonic ISO(7) supergravity and the duality hierarchy, J. High Energy Phys. 02 (2016) 079.

[75] A. Guarino and O. Varela, Consistent $\mathcal{N}=8$ truncation of massive IIA on $S^{6}$, J. High Energy Phys. 12 (2015) 020.

[76] O. Varela, $\mathrm{AdS}_{4}$ solutions of massive IIA from dyonic ISO (7) supergravity, J. High Energy Phys. 03 (2016) 071.

[77] N. Bobev, A. M. Charles, K. Hristov, and V. Reys, The Unreasonable Effectiveness of Higher-Derivative
Supergravity in $\mathrm{AdS}_{4}$ Holography, Phys. Rev. Lett. 125, 131601 (2020).

[78] S. M. Hosseini and A. Zaffaroni, Geometry of $\mathcal{I}$-extremization and black holes microstates, J. High Energy Phys. 07 (2019) 174.

[79] J. P. Gauntlett, D. Martelli, and J. Sparks, Toric geometry and the dual of $\mathcal{I}$-extremization, J. High Energy Phys. 06 (2019) 140.

[80] H. Kim and N. Kim, Black holes with baryonic charge and $\mathcal{I}$-extremization, J. High Energy Phys. 11 (2019) 050.

[81] N. Bobev and P. M. Crichigno, Universal RG flows across dimensions and holography, J. High Energy Phys. 12 (2017) 065.

[82] N. Bobev, A. M. Charles, and V. S. Min, Euclidean black saddles and $\mathrm{AdS}_{4}$ black holes, J. High Energy Phys. 10 (2020) 073. 